University of Louisville

ThinkIR: The University of Louisville's Institutional Repository

Electronic Theses and Dissertations

$5-2007$

\title{
Motivation and attitude of preservice elementary teachers toward mathematics : is rural revevant?
}

Christine Ankrom Perry

University of Louisville

Follow this and additional works at: https://ir.library.louisville.edu/etd

\section{Recommended Citation}

Perry, Christine Ankrom, "Motivation and attitude of preservice elementary teachers toward mathematics : is rural revevant?" (2007). Electronic Theses and Dissertations. Paper 1118.

https://doi.org/10.18297/etd/1118

This Doctoral Dissertation is brought to you for free and open access by ThinkIR: The University of Louisville's Institutional Repository. It has been accepted for inclusion in Electronic Theses and Dissertations by an authorized administrator of ThinkIR: The University of Louisville's Institutional Repository. This title appears here courtesy of the author, who has retained all other copyrights. For more information, please contact thinkir@louisville.edu. 


\title{
MOTIVATION AND ATTITUDE OF PRESERVICE ELEMENTARY TEACHERS TOWARD MATHEMATICS: \\ IS RURAL REVEVANT?
}

By

Christine Ankrom Perry

B. S. Morehead State University, 1975

M. A. Morehead State University, 1986

\author{
A Dissertation \\ Submitted to the Faculty of the \\ Graduate School of the University of Louisville \\ in Partial Fulfillment of the Requirements \\ for the Degree of
}

Doctor of Philosophy

Department of Teaching and Learning

University of Louisville

Louisville, Kentucky

May 2007 


\title{
MOTIVATION AND ATTITUDE OF PRESERVICE ELEMENTARY TEACHERS TOWARD MATHEMATICS: \\ IS RURAL RELEVANT?
}

$$
\text { By }
$$

Christine Ankrom Perry

B. S., Morehead State University

M. A., Morehead State University

$$
\text { A Dissertation Approved on }
$$

March 30, 2007

by the following Dissertation Committee

\author{
Dissertation Director
}




\section{ACKNOWLEDGMENTS}

The goal of achieving a doctoral degree has been an elusive one in my life. My success in reaching this goal is a result of the influence of many people and the confluence of many events. I would like to acknowledge those people that have guided me to and through this journey.

First, I would like to thank several members of my family. My mother, Helen Reed Ankrom, was my foremost inspiration. She taught me at a young age that women can continue to pursue their goals even after raising a family. She taught me this by example, and I have never forgotten this valuable lesson. My sister, Connie Ankrom Ray, served as my role model for attaining this degree. Also by example, she showed me what was possible, and was a valuable source of support and encouragement along the way. I also want to thank my daughters, Leslie and Allison, for their tolerant listening as I used them to test my thoughts and ideas. Finally, I would like to thank my husband, Byrd, for his love and patience as he spent untold hours alone while I followed my personal dream.

Second, my dissertation chair, Dr. Karen Karp, has been invaluable to me in completing this project. Her critiques and suggestions contributed enormously to the soundness of the final product, and her mentoring gave me the confidence to persevere. I feel fortunate to have had the benefit of her professional wisdom throughout this process. 
I would also like to thank the other members of my dissertation committee. Dr. Bill Bush and Dr. Alan DeYoung provided valuable input on this dissertation. I truly appreciate the time they contributed to read drafts and offer suggestions. A special thanks goes to Dr. Joseph Petrosko for his guidance with the research design and statistical analysis. His efforts and patience with my questions have contributed greatly to my growth as a researcher. I also want to thank Dr. Wiley Williams for providing some vital assistance in the data collection phase of this project.

Finally, I especially want to thank all the members of the ACCLAIM family, particularly Dr. Bill Bush and Dr. Vena Long. The members of my cohort have been an immeasurable source of support and encouragement. Thanks go to Caroline Best, Brian Boyd, Barbara Buckner, Frank Edge, Craig Green, Julianna Gregory, Debbie King, Brenda Lackey, Bill Maxson, Sue Nichols, Judy Pennington-Price, Crystal Rice, and Karla Willis. I cannot imagine completing a doctoral degree without the support system provided by this group of people.

There is no doubt that without the ACCLAIM project my goal of attaining a doctoral degree would have continued to elude me. I hope that in the coming years I can repay this debt of gratitude by working toward the project's goal of improving mathematics teaching and learning in K-12 schools and improving teacher education programs in the Appalachian region. 


\title{
ABSTRACT \\ MOTIVATION AND ATTITUDE OF PRESERVICE ELEMENTARY TEACHERS TOWARD MATHEMATICS: \\ IS RURAL RELEVANT?
}

\author{
Christine Ankrom Perry
}

May 12, 2007

This dissertation was a quantitative study with a sample of preservice elementary teachers from four universities in Kentucky. The purpose of this study was to investigate preservice elementary teachers' achievement goal orientations for learning mathematics and the relationship of those goals and their attitudes toward mathematics. A second goal of this study was to explore differences in the types of achievement goals and attitudes between rural preservice elementary teachers and their nonrural counterparts.

Self-report instruments were administered to assess the level of three achievement goals - mastery, performance-approach, and performance-avoid, and three constructs of attitude - confidence in learning mathematics, usefulness of mathematics, and mathematics as a male domain. The participants also completed a questionnaire designed to determine their rural/nonrural educational background. The sample was divided into four subgroups based on locale: Appalachian rural, other rural, urban, and other nonrural. 
Results indicated that preservice elementary teachers were significantly higher in mastery goals than in performance goals, and that performance-avoid goals were significantly higher than performance-approach goals. These preservice teachers were also less confident in learning mathematics than a sample of female students pursuing a variety of majors (Eckard, 1995). Mastery goals were weakly to moderately correlated to all three constructs of attitude. A statistically significant difference between the Appalachian rural group and the other nonrural group for confidence in learning mathematics was also found, with the Appalachian rural group displaying less confidence. Furthermore, rural preservice teachers had less confidence and were more likely to view mathematics as a male domain than nonrural preservice elementary teachers.

Since mathematics classes are traditionally performance-oriented, the result that preservice elementary teachers are high in mastery goals suggests a mismatch between personal and classroom goals that could result in negative attitudes toward mathematics and the adoption of maladaptive performance-avoid goals. The findings of this study suggest that mathematics educators teaching mathematics content courses for preservice elementary teachers from all locales should create a classroom climate that supports and encourages mastery goals. The characteristic of such a classroom align with the practices suggested by the NTCM Standards $(1989,1991,2000)$. 


\section{TABLE OF CONTENTS}

\section{PAGE}

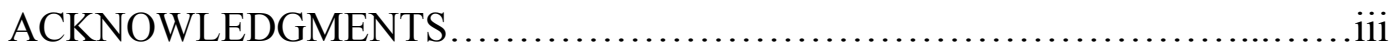

ABSTRACT ..........................................................

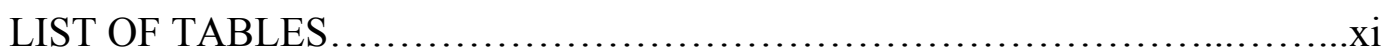

CHAPTER

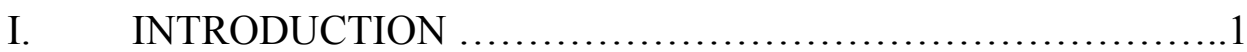

Theoretical Framework..............................4

Motivation.....................................4

Achievement motivation and goal theory....4

Intrinsic and extrinsic motivation..........6

Multiple goals...........................

Attribution theory.......................8

Expectancy $x$ value theory $\ldots \ldots \ldots \ldots \ldots . . . . .9$

Attitude......................................10

Cultural Issues.................................11

Statement of the Problem............................... 12

Purpose and Research Questions......................15

Hypotheses.......................................16

Significance of the Study.............................. 18

Limitations..........................................20 
Summary 21

Definitions...... 23

Organization of the Study ..............................26

II. REVIEW OF LITERATURE....................................27

Motivation............................................28

Research on Classroom Goal Orientation...........28

Research on Personal Goal Orientation...............37

Effect of the learning climate................37

Relation to academic performance............39

Relation to students' attitudes, beliefs and learning behaviors........................41

Research using the three-goal model.........44

Research on the pursuit of multiple goals...48

Summary .........................................

Attitude...............................................55

Student Attitudes................................55

Elementary Teacher and Preservice Teacher

Attitudes............................................62

Cultural Issues........................................66

The Rural Culture and Motivation..................71

The Appalachian Rural Culture.....................73

General Values and Attitudes...............74

Attitudes toward Schools and Education....78

Mathematics in Appalachia..................81 
Rural Elementary Teachers...................82

Summary........................................ 84

III. METHODOLOGY ........................................... 89

Design............................................ 89

Sample........................................90

Instrumentation...................................96

Procedures.......................................101

Data Analysis.....................................103

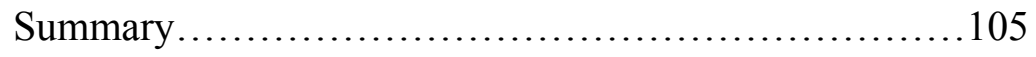

IV. RESULTS........................................... 107

Sample.........................................107

Reliability Analysis...............................109

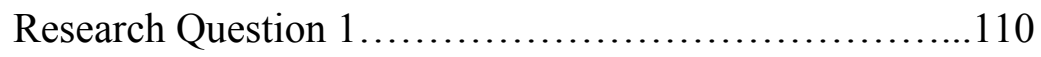

Research Question 2.............................112

Research Question 3.............................114

Research Question $4 \ldots \ldots \ldots \ldots \ldots \ldots \ldots \ldots \ldots \ldots \ldots \ldots \ldots \ldots \ldots$

Summary ...................................... 124

V. DISCUSSION, LIMITATIONS AND IMPLICATIONS..........127

Methodology.................................... 128

Limitations.....................................130 


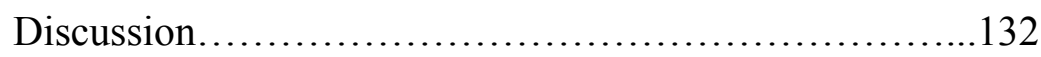

Goal Orientations..............................133

Attitudes....................................135

Attitudes and Goal Orientations.................136

Rural/Nonrural Differences......................139

Implications...................................140

Teacher Preparation...........................140

Future Research............................148

Summary......................................150

REFERENCES................................................ 153

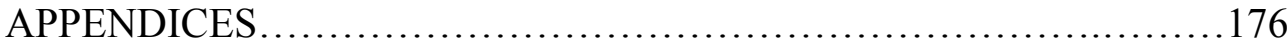

CURRICULUM VITAE......................................... 183 


\section{LIST OF TABLES}

TABLE $\quad$ PAGE

1. Variables Used in the Research...............................................99

2. Operational Definitions of Levels of the Independent Variable....................95

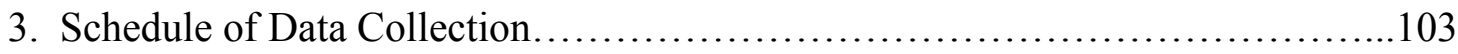

4. Reliability Coefficients for Scales............................................ 110

5. Descriptive Statistics for PALS Achievement Goal Orientation Scales.............111

6. Paired Samples t-Test Results for Comparisons of PALS Scales..................112

7. Comparative Statistics on Preservice Teachers and Female College Students from Eckard (1995) .................................................... 114

8. Correlations Among the Subscales for Achievement Goal Orientations and Attitudes.................................................................. 115

9. Correlations for the Rural group $(n=136)$ and the Nonrural group $(n=150) \ldots \ldots .117$

10. Descriptive Statistics for the Variables for the Four Locale Groups.................118

11. Multivariate Tests for MANOVA of Locale (Four Levels) Related to Achievement Goal Orientation and Attitude (Six Measures).....................119

12. Descriptive Statistics for the Variables for Two Locale Groups: Rural and

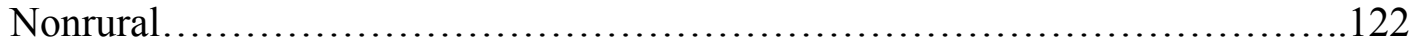

13. Multivariate Tests on Three Attitude Variables by Two Groups, Rural and Nonrural. 


\section{CHAPTER I \\ INTRODUCTION}

According to the results of international tests, the mathematics performance of students in the United States is comparatively weak on even the most basic of mathematical concepts (Elliott, Hufton, Illushin, \& Lauchlin, 2001; Stigler \& Hiebert, 1999). As a result, interest in K-12 mathematics education reform continues to build and attract a wider audience. Since 1989, the National Council of Teachers of Mathematics (NCTM) has stressed that all students should achieve at higher levels (NCTM 1989, 1991, 2000), and the Mathematical Association of America (MAA) now recognizes the challenge of these rising expectations for K-12 mathematics education (Conference Board of the Mathematical Sciences [CBMS], 2001). Additionally, in 2001 the United States Congress passed the No Child Left Behind (NCLB) Act as part of the President's education reform plan. This act requires that all children attain higher educational goals. Despite recent demands and more than fifteen years of efforts by NCTM to reform the way mathematics is taught in classrooms across the country, student performance continues to be disappointing.

Meeting the challenges of mathematics reform will require that we engage, or motivate, more students to learn more mathematics at deeper levels. While the construct of motivation is of considerable concern to education professionals in general, it is 
particularly important in the mathematics classroom (Gottfried, 1985, 1990; Grouws \& Lembke, 1996; Kloosterman \& Gorman, 1990; Middleton, 1992). The preponderance of questions related to motivation submitted to a panel of master teachers at a recent conference for preservice teachers sponsored by the Appalachian Collaborative Center for Learning, Assessment and Instruction in Mathematics (ACCLAIM) points to this concern. In fact, many psychologists choose to situate motivation research specifically in the mathematics classroom because the characteristics of many mathematics classrooms appear to facilitate maladaptive patterns of motivation (Ryan \& Patrick, 2001; Turner et al., 1998).

A focus on motivational issues provides another approach to implementing reform practices. Elementary teachers often do not view themselves as mathematics teachers and do not identity with organizations such as NCTM that seek to reform teaching practices. Because many elementary teachers dislike mathematics, they avoid professional development in this area (Meyer, 1980). However, they may see motivation as a more friendly and valuable topic; one they can master and benefit from. Fortunately, considerable alignment exists between the practices researchers indicate should be implemented to improve motivation in mathematics classrooms (Anderman, Patrick \& Ryan, 2004; Grouws \& Lembke, 1996; Meece, Blumenfeld, \& Hoyle, 1988; Middleton \& Spanias, 1999; Stipek et al., 1998; Wood \& Sellers, 1997) and the changes called for in the NCTM Standards $(1989,1991,2000)$.

This study will assess the motivational achievement goals of preservice elementary teachers in the area of mathematics. These college students are both students of mathematics and future mathematics teachers. The achievement goals they hold have 
implications for their own learning of mathematics content (Barron, Harackiewicz, \& Tauer, 2001; Urdan, Pajares, \& Lapin, 1997), and mastery of content knowledge affects their future teaching of mathematics (Ball, Lubienski, \& Mewborn, 2001; Ma, 1999).

Meyer (1980) claimed that in addition to poor content knowledge, many elementary teachers have negative attitudes toward mathematics. Attitudes are learned tendencies to react to an object in a consistently positive or negative manner (Fishbein \& Ajzen, 1975). Poor attitudes toward mathematics also adversely affect a teacher's instructional practices in mathematics (Karp, 1988; 1991). On the other hand, more reform-oriented instruction occurs in classrooms where teachers have positive attitudes towards mathematics (Wilkins, 2002). Teachers with positive attitudes toward mathematics are more likely to develop positive attitudes in their students (Banks, 1964). $\operatorname{NCTM}(1989,1991,2000)$ has recognized the importance of developing positive student attitudes towards mathematics.

Attitudes are important because they may affect learning. Aiken (1972a) argued that considering one variable of attitude could improve performance in mathematics. In a meta-analysis of the research, Ma and Kishor (1997) found a positive and reliable relationship between attitude and achievement in mathematics; however, the relationship was not strong. Research indicates that attitude is important from both the student and teacher perspective (Aiken, 1972a; Ma \& Kishor, 1997; Wilkins, 2002). Preservice teachers are in the unique position of transitioning from student to teacher. Improving their attitudes towards mathematics would certainly be a step toward meeting the rising expectations in K-12 mathematics education. 
Some research suggests that motivational issues relate to attitudes (Ames \& Archer, 1988; Seifert, 1995; Turner, Thorpe \& Meyer, 1998). For example, Ames and Archer found that students with mastery goals reported more positive attitudes toward their class. This proposed study will examine the relationship between motivation and attitude in preservice elementary teachers.

\section{Theoretical Framework}

\section{Motivation}

Motivation encompasses a multitude of factors that determine one's choice of activity (Dweck \& Elliott, 1983). According to Ames (1992) motivational factors exist as part of one's goal structures and beliefs about what is important. These factors determine whether one will participate in a given activity (Ames, 1992) and, therefore, exert tremendous influence on intellectual performance and achievement independent of one's ability (Dweck \& Elliott, 1983). Research in this area focuses on the reasoning processes, inference rules, and problem-solving strategies that individuals use under a variety of conditions. The complexity of this construct has led to a variety of theories and approaches to research.

\section{Achievement motivation and goal theory.}

Education professionals are generally interested in a type of motivation referred to as achievement motivation (Dweck \& Elliott, 1983). Achievement motivation of students depends on their goals related to competence. According to Dweck and Elliott, a student's goal may be to gain competence in some area, or the goal may be to achieve a favorable judgment of competence. Other students may seek to avoid unfavorable judgments as their goal. 
Motivational researchers have identified two major goal orientations. They refer to these orientations by a variety of names. The first orientation is the goal to gain competence, which is often labeled as either mastery (Ames \& Archer, 1988; Patrick, Anderman, Ryan, Edelin, \& Midgley, 2001; Turner et al., 2002), task (Anderman, Maehr, \& Midgley, 1999; Nicholls, Cobb, Wood, Yackel \& Patashnick, 1990; Ryan \& Patrick, 2001; Ryan \& Pintrich, 1997; Urdan, Midgley, \& Anderman, 1998; Wood \& Sellers, 1997), or learning (Stipek et al., 1998; Turner et al., 1998). The other orientation is the goal to be judged as competent, or not to be judged as incompetent. This type of goal is frequently labeled performance (Ames \& Archer, 1988; Anderman et al., 1999; Patrick et al., 2001; Stipek et al., 1998; Turner et al., 2002), ability (Ryan \& Patrick, 2001; Ryan \& Pintrich, 1997; Turner et al., 1998; Urdan et al., 1998), or ego (Nicholls et al., 1990; Wood \& Seller, 1997). The primary difference in these two types of goal orientations is whether learning is valued as an end in itself (mastery, task, learning), or if learning is just a means to reach some external goal (performance, ability, ego) (Meece et al., 1988).

With a mastery/task/learning goal orientation, the student's actual goal is to learn, and classrooms oriented to this goal will encourage students to master tasks and develop intellectually. Success from this perspective is attained through effort. On the other hand, a performance/ability/ego goal orientation is a perspective that values ability and holds the belief that ability involves reaching success with little effort. The goal of performance-oriented students is some objective other than learning such as winning a prize or getting high grades. These objectives are external to gaining knowledge (Lepper, 1988). Classrooms geared to this orientation are competitive and emphasize grades. Ames and Archer (1988) argued that the conceptual relationships among mastery, 
task, and learning goals are the same, and clustered them under one label - mastery goals. They made the same argument to cluster performance, ego, and ability goals into one label - performance goals. For the purpose of clarity, this study will adopt these two labels as well.

Performance goals may be further divided into two distinct categories labeled performance-approach and performance-avoid goals (Dweck \& Elliott, 1983). Students with performance-approach goals seek favorable judgments of their competence; while students with performance-avoid goals seek to prevent unfavorable judgments of their ability. Urdan et al. (1997) proposed that a lack of separation between these two dimensions of performance goals resulted in the weak relationships among performance goals and other variables found by some researchers. Results of a study by Elliot and Church (1997) supported the idea that performance-approach and performance-avoid goals are separate orientations with different effects. On the other hand, Middleton and Midgley (1997) maintained that the distinction between mastery and performance goals is the most important determiner of achievement behavior. However, they also found a relationship between performance-avoid goals and adverse learning behaviors such as avoidance of help-seeking. The present study will utilize the trichotomous framework of goal theory and investigate the mastery, performance-approach, and performance-avoid goals of elementary preservice teachers.

Intrinsic and extrinsic motivation.

Some theorists classify motivation related to academic settings as intrinsic or extrinsic (Middleton \& Spanias, 1999). However, Lepper (1988) maintains that these 
categories are broader than mastery and performance orientations, and can be applied to situations outside the domain of education or achievement.

Students who are intrinsically motivated will stay on task longer and persist when faced with difficulties (Boekaerts, 2002). Researchers describe intrinsically motivated students as students who like learning "for its own sake"; whereas students who do their academic work solely to obtain some external reward or avoid punishment are extrinsically motivated (Lepper, 1988; Middleton \& Spanias, 1999). Extrinsically motivated students are not concerned with superiority or judgments of their performance in relation to others. An external reward, such as a grade, or avoidance of punishment is what motivates these students.

Anderman et al. (1999) argued that performance goals and extrinsic goals are distinct. To measure performance goals, they assessed students' orientation to demonstrate ability related to others; but they assessed extrinsic goals as an orientation to grades and correct answers. Anderman et al. asserted that studies using samples of early adolescents have supported these as two separate factors (Midgley et al., 2000). However, Middleton and Spanias (1999) pointed out that extrinsically motivated students tended to have performance goals, and intrinsically motivated students lean towards mastery (learning) goals. In summary, some researchers use achievement goals such as mastery and performance to categorize motivation in education, and others use the intrinsic/extrinsic categories. This study will focus on mastery and performance goal orientation categories.

Multiple goals. 
Mastery and performance goals are not necessarily dichotomous. According to Dweck and Elliott (1983), children are not measured in terms of high or low achievement motivation; instead, a high or low value may be attached to various goals. Many researchers examine patterns of mastery and performance goals (Meece \& Holt, 1993; Turner et al., 1998) or look at the levels of mastery and/or performance goals exhibited in an experimental setting (Barron \& Harackiewicz, 2001). Most of this research was situated in public schools (Ames \& Archer, 1988; Meece \& Holt, 1993) or used college students in psychology and education classes as subjects (Barron \& Harackiewicz, 2001). This study will add to this body of literature by assessing the types of goals adopted by preservice elementary teachers in mathematics classes.

\section{Attribution theory.}

Another approach to research on motivation in mathematics education is attribution theory (Middleton \& Spanias, 1999). This theory attempts to explain student motivation based on their perceptions of the reasons for success or failure. According to Kloosterman (1984), students in mathematics classrooms are more aware of their success and failure than in other subjects because mathematics problems are usually clearly right or wrong. Students who attribute their success to causes under their control, such as effort, and their failures to uncontrollable sources, such as bad luck or an especially difficult task, are highly motivated. They believe they can succeed with effort, and since they control the amount of effort expended, success is within their reach. Students who believe that failure is due to lack of ability, which is not under their control, are poorly motivated. These students believe that since ability is stable, no amount of effort will result in success. They attribute any success they may achieve to task simplicity or luck. 
Attributional style is a classification of students based on how they explain their success or failure in school. Those who feel success is due to effort are labeled mastery-oriented, and those who believe effort is useless and will not lead to success are labeled learned helpless (Diener \& Dweck, 1978; Dweck, 1975; Kloosterman, 1988).

In goal theory, a performance goal orientation is a perspective that values ability, and a mastery orientation endorses effort; therefore, a likely relationship between goal theory and attribution theory is evident (Ames, 1984). Middleton and Spanias (1999) noted that students with mastery (learning) goals tended to believe success is a result of hard work, collaboration and seeking to understand; whereas, those possessing performance (ego) goals defined success through social comparison and asserting their superiority.

Expectancy $x$ value theory.

Another theory that sheds light on poor motivation to learn mathematics is expectancy x value theory (Good \& Brophy, 1991; Grouws \& Lembke, 1996). This theory contends that motivation is the product of two factors: (a) The expectations for success in given task, and (b) the degree that the rewards of success are valued. Since this is a product, if either factor is zero there is logically no motivation to expend effort on the task. Students will not spend effort on a task if they do not value the outcomes, even if they have confidence in success. Furthermore, students will not spend effort if they do not expect to be successful, even if they value the outcomes of success. Since a greater number of people have low expectations of success in mathematics than in any other subject (Grouws \& Lembke, 1996; Kloosterman \& Gorman, 1990), the expectancy $\mathrm{x}$ value theory portends serious motivational problems in learning mathematics. 
Attitude

According to Fishbein and Ajzen (1975) an attitude is "a learned predisposition to respond in a consistently favorable or unfavorable manner with respect to a given object" (p. 6). They argue that affect is the most important part of attitude. Affect refers to the feelings one has toward some object or one's evaluation of the object. Therefore, attitude refers to a person's favorable or unfavorable feelings toward or evaluation of an object. A significant part of their definition is that attitudes are learned. Whether a person responds favorably or unfavorably toward an object is a result of past experiences with that object.

Fishbein and Ajzen (1975) presented a conceptual framework that used beliefs as the fundamental building blocks of attitude. They contend that a person's attitude is a function of their beliefs. Beliefs represent the information one has accumulated through past experience with the object. Therefore, as individuals accumulate beliefs about a specific object, they form an attitude toward that object at the same time. While numerous beliefs may accrue about an object, only a small number will determine attitude at a particular point in time. Fishbein and Ajzen refered to these as the salient beliefs. According to their theory, attitudes that result from salient beliefs were one determinant of a person's intention to perform a specific behavior, but there are other determinants as well. Therefore, attitude does not necessarily predict behavior, but predicts the intention to perform some behavior.

Beliefs vary in strength, and individuals evaluate their beliefs according to the attributes associated with that belief (Fishbein \& Ajzen, 1975). Beliefs associated with positive attributes are evaluated positively, and those associated with negative attributes 
are rated negatively. Fishbein (1963) argued that an attitude is the summation of the products of salient belief strength and evaluation. His model indicates that this summation will result in an attitude toward the object that will be either positive or negative.

Fishbein's (1963) theory implies that attitude toward mathematics is a result of one's past experiences with mathematics and the salient beliefs that have developed through those experiences. Mastery-oriented and performance-oriented students have different beliefs. For example, mastery-oriented students believe that that failure is due to lack of effort, but performance-oriented students believe that failure is due to lack of ability (Diener \& Dweck, 1978).

\section{Cultural Issues}

Variation in personal beliefs may contribute to the adoption of different types of achievement goals and attitudes toward mathematics, and cultural contexts help form an individual's beliefs. Parents from different cultures pass their beliefs about achievement on to their children (Stevenson, Lee, Chen, Stigler, Hsu \& Kitamura, 1990).

Additionally, societal attitudes specific to mathematics may result in motivation in mathematics that is different from motivation in other subject areas (Carr, 1996).

This study will compare the achievement goals and attitudes of preservice elementary teachers from nonrural areas with those from rural areas, particularly the rural Appalachian region of Kentucky. This region wrests with the value of education (Howley, 2003; Elliott, Hufton, Hildreth, \& Illushin, 1999; Elliott, Hufton, Illushin, \& Lauchlan, 2001). In cultures where education, particularly mathematics education, is not 
highly valued, the challenge of motivating students to excel in this subject area is particularly daunting.

Evidence from research indicates that the some Appalachian people do not appreciate education. For example, DeYoung (1995a, 1995b) found that some citizens in this region did not support local efforts to increase graduation rates and encourage college attendance. Ismail (2006) reported that many rural, low-income communities were not concerned with sanctions issued by the federal No Child Left Behind (NCLB) law. Other research reveals that students in the Appalachian region of eastern Kentucky place a lower value on education than students from other cultures. According to Elliott et al., (1999) and Elliott et al. (2001) students in eastern Kentucky stressed qualifications for work as a reason to study hard in school as opposed to Russian students who stressed the value of being an educated person. Results that eastern Kentucky students spend less time on homework, engage in fewer cultural pursuits, and spend less time reading than students in other countries are indicative of their lower appreciation for education. The reason for these differences may lie in cultural attitudes toward academic endeavors that are passed on from generation to generation.

According to Howley (2003) there has been very little attention in research toward influences in the rural culture that support mathematical learning or create resistance to instruction in mathematics.

\section{Statement of the Problem}

Students' motivation and attitudes toward mathematics develop early and are strongly influenced by teacher actions and attitudes (Aiken, 1972a; Karp, 1988, 1991; Middleton \& Spanias, 1999; Nicholls et al., 1990). Some studies suggest that 
motivational patterns are learned, and that students learn to like or dislike mathematics (Meece, Wigfield, \& Eccles, 1990; Midgley, Feldlaufer, \& Eccles, 1989). They also report that teachers unconsciously influence their students' motivation by imposing their own attitudes, beliefs, and motivations toward mathematics upon their students. Students pick up unintended messages from teachers about what it means to know and do mathematics (Karp, 1988, 1991; Kloosterman, Raymond, \& Emenaker, 1996). Additionally, teachers' ideas about what makes mathematics motivating play a significant role in the instructional activities that teachers choose (Middleton, 1992).

Teachers often model their mathematics teaching after their own former high school and elementary teachers (Ball et al., 2001; Brown \& Smith, 1997; Middleton, 1992). Unfortunately, university coursework does little to change beliefs shaped by $13+$ years of first hand experience as students in mathematics classrooms (Ball et al., 2001; Foss \& Kleinsasser, 1996). In addition, Hiebert, Gallimore, and Stigler (2002) noted that teachers seldom turn to research to guide their teaching practice. The hours of observations one accumulates as a student instill a view of teaching that is exceedingly difficult to change (Ball, 1988; Taylor, 2002). Preservice elementary teachers have spent many hours as students in mathematics classes using traditional instructional approaches (Ball, 1988) that tend to favor males (Fennema, 2000). Traditional instruction in mathematics is performance-oriented and encourages the adoption of performance goals (Anderman et al., 1999; Nicholls et al., 1990). Since most preservice elementary teachers are female, this type of instruction coupled with an initial inclination to mastery goals may negatively affect their attitude toward mathematics in their classroom and encourage the adoption of maladaptive performance goals by their students in the mathematics 
classes they teach. Thus, there is a cycle of female elementary teachers with negative attitudes toward mathematics unwittingly teaching mathematics in a traditional performance-oriented manner. As a result, these negative attitudes and performance goals in mathematics classes are passed on to mastery-oriented young girls who are the future female elementary teachers. This "hidden" cycle is particularly deleterious.

The focus of this research is to describe the types of achievement goals adopted by preservice elementary teachers with respect to instruction in mathematics. This study also will investigate the relationship between the types of goals that they adopt and their attitudes toward mathematics.

A particular cultural context may also be a contributing factor in the motivation of students to learn mathematics. According to Hart and Allexsaht-Snider (1996), consideration of the sociocultural circumstance is important in understanding the relationship between students' motivation and success in mathematics. Elliott et al. (1999) contend that sociocultural factors have not received adequate consideration in efforts at education reform. Their findings suggest that cultural influences may be more important than ability attributions in determining achievement goals. Additionally, Ball et al. (2001) list "culturally embedded views of knowledge, learning, and teaching" (p. 435) as a recurrent explanation for the failure of mathematics reform efforts.

The rural culture is a lifeworld in which people share common qualities such as land ethic, community, family, conservatism, and intradependence (Howley, 2003). These qualities tend to generate people who are communal, rather than individualistic, (Howley, 2003; Theobald, 1997). Since performance goals may be a by-product of an 
individualistic society (Hyde \& Kling, 2001), the communal disposition evident in rural cultures may affect student motivation to learn mathematics.

This study seeks to determine if there are differences in the types of motivational goals and attitudes toward mathematics between rural and nonrural preservice elementary teachers. If differences exist, then alternative strategies for preparing these future teachers may be warranted. Additionally, these preservice teachers are products of the rural and nonrural regions from which they came. While it would be inappropriate to generalize the results of this research to all rural and nonrural students, any differences found between these groups may point to areas that need further research in the more general rural student population. The results of this study will have implications for educators and policymakers who seek to maximize all students' motivation to learn mathematics.

\section{Purpose and Research Questions}

One purpose of this study is to investigate elementary preservice teachers' achievement goal orientations for learning mathematics and the relationship between those goals and their attitudes toward mathematics. The motivational goals and attitudes of preservice elementary teachers not only affect how well they learn mathematics as students themselves, but also how they will eventually teach mathematics to their own students. A second goal of this study is to explore differences in the types of motivational goals and attitudes between rural and nonrural preservice elementary teachers. Identification of cultural differences may lead to diverse strategies for improving the mathematics education of future elementary teachers and the subsequent mathematics education of their students. 
To study these issues, I propose the following questions for research.

1. What are the levels of achievement goal orientations - mastery goal orientation, performance-approach goal orientation, and performance-avoid goal orientation - for learning mathematics for preservice elementary teachers?

2. What are the attitudes of preservice elementary teachers toward mathematics as revealed by three measures of attitude: confidence in learning mathematics, usefulness of mathematics, and mathematics as a male domain?

3. What relationships exist, if any, among three measures of achievement goal orientation and three measures of attitude toward mathematics for preservice elementary teachers?

4. Do rural and nonrural preservice elementary teachers differ with respect to goal orientation for learning mathematics and attitudes toward mathematics?

Hypotheses

This research centers on the achievement goals and attitudes toward mathematics adopted by preservice elementary teachers in rural and nonrural regions of Kentucky. The following hypotheses are proposed for the research questions in this investigation:

1. What level of achievement goal orientations - mastery goal orientation, performance-approach goal orientation, and performance-avoid goal orientation - for learning mathematics are adopted by preservice elementary teachers?

Hypothesis: The mean scores for preservice elementary teachers' achievement goals will be highest in performance-avoidance goals, followed by mastery goals and lowest in performance-approach goals. 
2. What are the attitudes of preservice elementary teachers toward mathematics as revealed by three measures of attitude: confidence in learning mathematics, usefulness of mathematics, and mathematics as a male domain?

Hypothesis: Compared to published data on the instruments, the means for preservice elementary teachers will indicate low confidence in learning mathematics and a tendency to see mathematics as a male domain, and will indicate that they do not perceive mathematics as useful.

3. What are the relationships among three measures of achievement goal orientation and three measures of attitude toward mathematics for preservice elementary teachers?

Hypothesis: There will be a positive relationship between mastery goals and confidence. There will be a negative relationship between mastery goals and perception of mathematics as a male domain. There will be a negative relationship between performance-avoidance goals and confidence. There will be no relationship between performance-approach goals and constructs of attitude.

4. Do rural and nonrural preservice elementary teachers differ with respect to goal orientation for learning mathematics and attitudes toward mathematics?

Hypotheses: Rural preservice elementary teachers will have higher levels of mastery goals than nonrural preservice elementary teachers will. Rural preservice elementary teachers will report higher levels of confidence, and will be less likely to perceive mathematics as a male domain than nonrural preservice elementary teachers will. Rural preservice elementary teachers will perceive mathematics to be less useful than nonrural elementary teachers will. 


\section{Significance of the Study}

The overwhelming majority of elementary teachers and preservice elementary teachers are female. Results of some research suggest that females are more masteryoriented than males. For example, Fennema (2000) and Peterson and Fennema (1985) found that competition tends to favor male learning and has a negative affect on females, while cooperative activities are more conducive to female learning. Anderman et al. (1999) found that males were more performance-oriented than females. Moreover, Elliot and Church (1997) found a significant effect of gender indicating that women were more likely than men to adopt mastery goals. Since most mathematics classrooms tend to be performance oriented (Fennema, 2000; Turner et al., 1998), this mismatch between teachers' tendency toward mastery goals and the performance-oriented classroom climate may negatively affect their attitude toward mathematics and encourage them to adopt performance goals in mathematics classrooms.

Several studies provide support for this assertion. Harrackiewicz and Elliot (1993) argued that mastery-oriented students might experience anxiety in performanceoriented settings. Ball (1988) reports that many preservice teachers report previous negative experiences with mathematics. Bowd and Brady (2003) found that female preservice teachers reported more negative experiences with mathematics than male preservice teachers reported. Identifying the sources of negative attitudes is the first step in finding out why many elementary teachers dislike mathematics (Meyer, 1980). This study is a first step toward determining if a relationship between goal orientations and attitudes in mathematics exists. 
Teachers tend to teach mathematics in the manner that they were taught (Ball et al., 2001). Since most mathematics classrooms are performance-oriented (Fennema, 2000), elementary teachers are also likely to create a mathematics classroom climate that is perceived as being performance-oriented by their students. Thus, there is a cycle of female elementary teachers with negative attitudes toward mathematics teaching young girls, who are initially mastery-oriented and who may be the future female elementary teachers, using performance-oriented instructional approaches.

While several researchers have investigated the achievement goals of college students (Archer, 1994; Barron \& Harackiewicz, 2001; Barron, Harackiewicz, \& Tauer, 2001; Harackiewicz, Barron, Tauer, Carter, \& Elliot, 2000; Karabenick, 2004; Magnusson \& Perry, 1992), none has specifically examined the mathematics achievement goals of preservice elementary teachers. Knowledge of the motivational goals of these students could assist mathematics educators in providing effective mathematics instruction that will also improve the attitudes of these future teachers toward mathematics. Use of mastery-oriented instructional strategies in elementary mathematics content courses may provide a model of teaching that preservice teachers can use in their own future classrooms. These mastery-oriented teaching practices tend to be consistent with those advocated by NCTM (Stipek et al., 1998). Furthermore, enhancing teacher attitudes may encourage the use of more reform-oriented instruction in the classroom (Wilkins, 2002).

A few early studies suggest that nonrural schools provide more adaptive motivational climates than rural schools do (Jones \& Taylor, 1963; Randhawa \& Michayluk, 1975); however, others argue that rural schools have improved greatly in the 
last four decades (Kannapel \& DeYoung, 1999). Freeman and Anderman (2005) found that rural students tend to have higher levels of mastery goals than their nonrural counterparts; however, their study was not specific to mathematics. If their results hold for mathematics, then the mismatch between the personal mastery goals of the students and the performance goal structure of traditional mathematics instruction may be particularly acute.

This study will seek to determine if differences in goal orientations in the mathematics classroom exist between rural and nonrural preservice elementary teachers. Different goal orientations point to alternative strategies for teaching and motivating students to learn.

Additionally, if differences exist in the context of mathematics instruction, then future research is needed in this area on other rural student populations. If rural students have higher levels of mastery goals than nonrural students do, and nonrural students have higher levels of performance goals than rural students do, then results would indicate that performance-oriented motivational strategies might be effective in nonrural areas, but ineffective and possibly detrimental to rural areas. For example, slogans such as the Kentucky Department of Education's “Education Pays" may be counterproductive for rural regions of the state. This slogan may be interpreted as an individualistic, urban model appealing to those with high levels of performance goals. Its promise of monetary reward for academic effort is insubstantial at best for those living in rural areas of the state. For mastery-oriented rural students in the Appalachian region of Kentucky, this slogan may be less effective.

\section{Limitations}


This study will investigate the goal orientations and attitudes of preservice teachers at colleges and universities in an Appalachian state in the Midwest. The sample for this study will be a sample of convenience and will not be random. Therefore, any generalization of the results will be limited.

This study also relies heavily on definitions of rural and nonrural schools that will be used to subdivide the participants into four locale groups. Defining rural is a problem for all rural researchers (U. S. Department of Education, 1994). Compounding the problem is that not all rural cultures are the same (Bush, 2003). Selecting a representative sample from the rural culture one wishes to study is one of the most difficult tasks for a rural researcher. Any generalization of these results will need to employ the same definitions utilized by this study.

All of the instruments used in this study are self-report measures. It is possible that participants will respond in a manner they believe to be acceptable rather than answering frankly and honestly. According to Sax (1989), these measures "are susceptible to response biases, easily faked, difficult to interpret, and tend to be unreliable (p. 521)" when compared to cognitive measures.

This researcher teaches the first mathematics content course at one of the universities in the sample. As a result, some of the study participants may be her students. A colleague of the researcher will administer the survey to insure anonymity so these students feel no coercion to participate; however, their openness in responding to the survey questions could be affected.

Summary 
Mathematics educators at all educational levels are challenged with the task of improving student learning in mathematics. Student motivation to learn mathematics is a significant concern for meeting this challenge (Gottfried, 1985, 1990; Grouws \& Lembke, 1996; Kloosterman \& Gorman, 1990; Middleton, 1992).

Teacher actions and attitudes towards mathematics strongly influence the motivations and attitudes of their students (Karp, 1988, 1991; Middleton \& Spanias, 1999). Teachers tend to teach mathematics in the manner they were taught (Ball et al., 2001; Brown \& Smith, 1997; Middleton, 1992), and most were taught using traditional approaches (Ball, 1988) that are performance-oriented and tend to favor males (Fennema, 2000). However, research indicates that females are inclined to mastery goals (Fennema, 2000; Peterson \& Fennema, 1985). The result is a possible mismatch between the achievement goals of preservice elementary teachers and the instructional approach used in the traditional mathematics classroom that may adversely affect their attitude towards mathematics, and encourage them to adopt maladaptive performance goals in mathematics classrooms. This study will investigate the relationship between the achievement goals and attitudes of elementary preservice teachers toward mathematics.

Cultural influences may also affect mathematics learning (Ball et al., 2001; Elliott et al., 1999; Fennema, 2000; Hart \& Allexsaht-Snider, 1996). Instructional approaches may not work equally well with both rural and nonrural students due to these cultural differences. There is some evidence that rural students have higher levels of mastery goals than their nonrural counterparts (Elliott et al., 2001; Freeman \& Anderman, 2005). This study seeks to determine if these results hold for preservice elementary teachers in the mathematics classroom. 


\section{Definitions}

Achievement goals : These are goals related to competence, and there are two distinct types. Mastery achievement goals center around learning and developing competence, while performance goals focus on performing or demonstrating competence (Dweck \& Elliott, 1983).

Appalachian rural preservice teacher: A preservice teacher who attended Appalachian rural schools for at least 8 of their 13 years of K-12 schooling.

Appalachian rural school: A rural school located in an Appalachian county.

Approach to instruction: There are a number of teacher practices that have been observed in both the cognitive psychology literature and the mathematics reform literature to affect motivation in the mathematics classroom. A number of these practices can be classified as either promoting a mastery-goal orientation or a performance-goal orientation in students. Mastery approaches are practices that encourage student mastery goals. These teaching strategies communicate to students that developing competence is the reason for participating in academic work (Midgley et al., 2000). Performance approaches are methods that encourage a performance-goal orientation in students. These practices indicate to students that demonstrating competence is the objective for academic work (Midgley et al, 2000).

Attitude: A consistently favorable or unfavorable reaction to a specific object. These reactions are learned tendencies that utilize abilities and past experience (Fishbein \& Ajzen, 1975).

Classroom goal orientation - This refers to the approach to instruction exhibited in a particular classroom. A performance-oriented classroom is a classroom that shows 
evidence of a performance approach to instruction, and a mastery-oriented classroom demonstrated a mastery approach to instruction.

Extrinsic motivation: This type of motivation occurs when the decision to engage in an activity is based on something that is external to the activity (Sansone \& Harackiewicz, 2000).

Intrinsic motivation: This motivation occurs when an activity satisfies basic human needs for competence or control. This motivation makes the activity interesting to the person and therefore she will engage in the activity for its own sake rather than as a means to an end (Lepper \& Henderlong, 2000).

Nonrural preservice teacher: A preservice teacher who spent at least eight years as a student in nonrural schools completing grades K-12.

Mastery goal orientation: An orientation towards learning that promotes the value of learning for its own sake. Students with these goals want to develop their competence and increase their understanding (Midgley et al., 2000).

Other nonrural school: A school to which the National Center for Educational Statistics (NCES) assigned one of the following locale codes: 3-Urban fringe of large city, 4Urban fringe of mid-size city, or 5 - Large town. For the purpose of this study, schools given the code " 6 - Small town" are also considered nonrural if the small town has a population of 10,000 or more.

Performance goal orientation: This is a learning perspective that values ability. Students with these goals want to demonstrate their competence. These goals can sometimes be adaptive and sometimes maladaptive (Midgley et al., 2000). Students with performance goals may be further divided into those with performance-approach goals and those with 
performance-avoid goals. A student with performance-approach goals seeks favorable judgments of her competence. A student with performance-avoid goals seeks to avoid unfavorable judgments of competence.

Motivations: Motivations are the reasons one has for behaving in a particular way in a given situation (Middleton \& Spanias, 1999).

Preservice elementary teacher: A college student enrolled in the first mathematics content course required for a program of study leading to certification in elementary education. This definition differs from the usual definition because this course is generally required as a prerequisite to admission to a teacher education program. Other rural preservice teacher: A preservice teacher who spent at least eight years as a student in rural schools outside of Appalachia completing grades K-12.

Other nonrural preservice teacher: A preservice teacher who spent at least eight years as a student in other nonrural schools completing grades K-12.

Other nonrural school: A school to which the National Center for Educational Statistics (NCES) assigned one of the following locale codes: 3 - urban fringe of a large city, 2 urban fringe of a mid-size city, 5- large town, or 6 - small town, if the town has a population of 10,000 or more.

Other rural school: A rural school located that is not located in Appalachia.

Rural school: A school to which the National Center for Educational Statistics (NCES) assigned one of the following locale codes: 7-Rural, outside CBSA/MSA or 8-Rural, inside CBSA/MSA. For the purpose of this study schools which were assigned the code "6 - Small Town" are also considered rural if the small town has a population less than 10,000 . 
Urban preservice teacher: A preservice teacher who spent at least eight years as a student in urban schools completing grades K-12.

Urban school: A school to which the National Center for Educational Statistics (NCES) assigned one of the following locale codes: 1-Large city, or 2-Mid-size city.

$$
\text { Organization of the Study }
$$

This study is organized into five chapters. Chapter 1 introduces the study and provides some background on motivational research, attitudes and rural issues. Chapter 2 is a review of literature relevant to this study. This includes research on motivation, attitudes toward mathematics, and rural issues. Chapter 3 explains the methodology used, which is a quantitative method utilizing self-report measures. Chapter 4 contains the results of the study, and chapter 5 consists of conclusions, implications, and possibilities for future research in this area. 


\section{CHAPTER II}

\section{REVIEW OF LITERATURE}

Prudence requires that a serious examination of the complexity of motivation in the mathematics classroom should begin with a definition. Dweck and Elliot (1983) offer the following summarizing definition of general motivation:

...the contemporaneous, dynamic psychological factors that influence such phenomena as the choice, initiation, direction, magnitude, persistence, resumption, and quality of goal-directed (including cognitive) activity. (p. 645)

Stated somewhat more succintly, "...motivations are the reasons individuals have for behaving in a given manner in a given situation" (Middleton \& Spanias, 1999, p. 66). Note that both of these definitions agree that motivation is not a single construct; but consists of a multitude of "factors" or "reasons" influencing a variety of goal-driven actions. Many theories and approaches to research in this area have resulted from the complexity of this construct. McLeod (1992) described motivation research as "diffuse and disconnected" (p. 586) because of the plethora of theories, approaches, and germane variables. Many researchers lament the fact that there is still no generally accepted theoretical framework to guide motivation research (McLeod, 1992; Middleton \& Spanias, 1999). Like a toddler, motivation research runs in different directions and constantly gets into trouble (Middleton \& Spanias, 1999).

This chapter reviews the extant literature related to motivation, attitude, and rural issues relevant to this research. The studies on motivation approach the concept from 
different perspectives. Some researchers focus on how the learning environment created by the teacher affects student motivation to learn (e.g. Ames, 1984; Ames \& Archer, 1988; Turner et al., 2002; Urdan et al., 1998). These studies are germane to pedagogy as teachers maintain considerable control over their own classroom climate. Other research concentrates on the personal motivational orientations of students (e. g. Aiken, 1972b, Anderman et al., 1999; Barron \& Harackiewicz, 2001; Nicholls et al., 1990; Urdan, Pajares, \& Lapin, 1997; Wood \& Sellers, 1997). These studies focus on the effect that adopting a particular goal orientation has on student attitudes, beliefs, and behaviors.

The attitudinal research selected for this review targets attitudes toward mathematics specifically, and much of it focuses on gender issues (e. g. Fennema \& Sherman, 1976a, 1978; Karp, 1988; 1991; Kloosterman, 1988; Ma \& Kishor, 1997; McMillan, 1976). This research is particularly salient to the present study because elementary teachers are predominantly female.

Finally, since this study will focus on how rural preservice elementary teachers differ from nonrural preservice elementary teachers, research on rural issues that incorporate motivation, attitudes toward mathematics, and elementary teachers will be reviewed.

\section{Motivation}

\section{Research on Classroom Goal Orientation}

The National Council of Teachers of Mathematics emphasized the importance of the learning environment in teaching mathematics by devoting an entire standard in its Professional Standards for Teaching Mathematics (NCTM, 1991). Standard 5: Learning Environment elaborates on the type of classroom climate teachers should create to 
promote students' mathematical power. The climate established by the teacher forms a hidden curriculum that communicates to students what it means to do and learn mathematics. Likewise, goal theory calls attention to the importance of examining the classroom climate for its influence on individual student motivation (Hart \& AllexsahtSnider, 1996).

A mastery learning orientation promotes the value of learning for its own sake. Classrooms oriented to this goal will encourage students to master tasks and develop intellectually. Success from this perspective is attained through effort (Middleton \& Spanias, 1999). This is consistent with NCTM's position that the primary focus of the learning environment must be on sense-making. Students must be allowed time to think, reason, and participate in mathematical discourse. They must feel safe to ask questions, try alternative approaches, and even to get stuck on a problem (NCTM, 1991). These activities encourage mastery and intellectual development.

A performance goal orientation is a perspective that values ability and holds the belief that ability involves reaching success with little effort. Classrooms with this orientation are competitive and emphasize grades (Middleton \& Spanias, 1999). Kumar (2004) asserts that performance-oriented classroom environments are counterproductive to students' progress in school. Kumar argues that children will not become lifelong learners if teachers immerse them in competitive classrooms and teach that the goal of education is getting good grades or high scores on standardized tests.

In 1984, Carol Ames conducted an experimental study designed to determine if the goal structure of a learning environment affected children's attributions for success or failure. She gave children puzzles to solve and manipulated the goal structure by varying 
the instructions she gave to the children. Some instructions emphasized competition, such as "Let's see who is better at solving the puzzles," and other instructions were challenging, such as "Try to solve as many puzzles as you can." Ames found that children focused more on ability attributions in a competitive setting and effort attributions in the setting emphasizing personal challenge and mastery. In other words, in the performance-oriented climate children focused on the question "Was I smart?" whereas, in the mastery setting they were thinking about "How can I do this task?" Four years later Ames and a colleague, Jennifer Archer, investigated how students' perceptions of classroom goals related to learning strategies, task preferences, attitudes, and causal attributions (Ames \& Archer, 1988). This study was important because it brought goal-orientation research out of the laboratory and into the classroom. The study involved 176 students in grades 8-11 in an academically advanced junior/senior high school. Four to six students from each class of English, mathematics, science and social studies responded to a questionnaire designed to determine their perceptions of the goal orientation of their class. They answered additional questions designed to assess their learning strategies (information processing, self-planning, and self-monitoring), preference for easy or challenging tasks, attitudes towards the class, causes of success or failure, and their perceived ability in that subject area compared to other students in the class. Ames and Archer found that when students perceived that the classroom orientation was on mastery goals, they reported using more learning strategies, preferred challenging tasks, and had a more positive attitude. Students attributed their success to effort, good study strategies, and the teachers. They did not consider ease of task to be a cause of success and seldom blamed their failures on the teacher. When 
students perceived that the classroom orientation was on performance goals, there was no significant correlation to learning strategies or task challenge. There was, however, a small negative correlation to attitude toward the class and to self-perceptions of ability. They also viewed effort as a cause of success, but the correlation was not as strong as under the perception of the classroom as one of mastery. Students in these classrooms attributed failure to lack of ability and work that was too difficult. These findings supported the results of Ames' 1984 experimental study. Overall, this research showed that a mastery-goal orientation in a classroom may result in sustained student involvement in learning and that students will pursue tasks that support increased learning.

A number of researchers have since studied how classroom goal orientation affects a number of other variables (Turner et al. 2002; Urdan et al., 1998). Using survey measures, Turner and others found that if students perceived an emphasis on performance goals, they participated in more self-handicapping strategies. Academic selfhandicapping strategies are avoidant behaviors such as not trying in academic work, not studying for a test until the last moment, or wasting time on unrelated activities the night before a test. Using these avoidance behaviors allows the student to blame poor performance on these behaviors rather than lack of ability. Conversely, Urdan and others (1998) found that avoidance behaviors were not related to perceptions of a performance goal structure. However, they also reported that students in mastery-oriented classrooms used avoidance strategies significantly less than those in performance-oriented classrooms. These findings support other research that suggests that the presence of a 
mastery classroom goal structure fosters more adaptive patterns of learning independent of performance goals (Ames \& Archer, 1988).

Additionally, Turner et al. (2002) investigated the nature of teacher discourse in classrooms where student perception of a mastery goal structure was identified as high and low. In perceived high-mastery classrooms, teacher discourse included a strong emphasis on learning, frequent encouragement, collaboration, encouraging intrinsic interest in mathematics, and recognition with genuine praise. These teachers used explicit statements that communicated to the students that they were competent learners. They also frequently used humor in a manner that was not distracting to the lesson. In contrast, teacher discourse in the perceived low-mastery classrooms rarely used humor and offered much less motivational support.

Other teacher practices also communicate the classroom goal structure to students. Patrick et al. (2001) used surveys and observational data to categorize classrooms by their goal structures and to describe teacher discourse and practices found in each category. Through this process, they identified specific teacher practices associated with classrooms that students perceived as having high levels of mastery goals and high levels of performance goals. In high-mastery classrooms, teachers ensured participation by all students, emphasized effort, and promoted student collaboration. Interestingly, they found that all teachers publicized performance, but the high-mastery teachers did so in a manner that did not implicate effort or ability. Teachers in classrooms perceived as low in mastery goals conveyed this performance information in a manner that also communicated expectations about success and exertion of effort. Patrick and her colleagues found similar results with the high and low performance 
groups. Both groups conveyed performance information and used rewards, but teachers in classrooms perceived as being low in performance goals did not associate assessments as being indicative of students' relative ability or future performance. Teachers in highperformance classrooms made these associations. Another interesting finding was that teachers in perceived high-mastery classrooms exhibited affective concern and support for students' learning and progress. This finding revealed that providing a warm and nurturing environment was not sufficient to communicate learning goals to students. Positive expectations for learning are also essential.

Ryan and Patrick (2001) investigated how various aspects of the classroom social environment related to changes in student motivation. This study involved 233 students and 15 teachers. The researchers controlled for student motivation in the seventh grade and looked at how the students' perceptions of their eighth grade mathematics classroom's social environment affected their motivational behaviors. The participants completed surveys to measure perceptions of classroom social environment and their motivation in the seventh grade and again in the eighth grade. The surveys contained items on the following aspects of the classroom environment: teacher support, student interaction, promotion of mutual respect, and promotion of performance goals. The survey items targeted motivational variables that included academic efficacy, social efficacy, self-regulated learning, and disruptive behavior. The results indicated that the classroom social environment explained changes in all of these motivational variables. In particular, researchers found that students' perceptions of their teacher as being supportive were important to their confidence relating to that teacher, their self regulated learning, and their behavior. Interestingly, these results indicated that when students 
were encouraged to interact with each other on academic tasks, they did not become more disruptive. This finding is notable because teachers are often reluctant to let students collaborate for fear that students will misbehave. Their study also supported the assertion that mutual respect is an important factor in predicting changes in academic efficacy and self-regulation of learning. Lastly, they found that when competition among students was encouraged, disruptive behavior was higher, and students reported less confidence in relating to their teacher. These results support the recommendations of mathematics reform educators pertaining to teacher practices relating to the learning environment as described in the Standard 5: Learning Environment, of the NCTM Professional Standards for Teaching Mathematics (NCTM, 1991). From a constructivist point of view, support, mutual respect, and trust must be a part of the classroom environment in order for genuine teaching and learning to occur (Kamii, 1994).

Stipek and others (1998) recognized this relationship noted by motivational researchers and the teaching practices advocated by mathematics education reformers. They conducted a study to assess the connections among reform teaching practices, student motivation, and learning mathematics. The motivational variables were focusing on learning, self-confidence, willingness to take risks and approaching challenging tasks, enjoyment, and positive feelings. The participants were 24 teachers and 624 students in several different school districts. The districts were in a large ethnically diverse urban area. Stipek and her colleagues videotaped teachers during instruction on addition of fractions and observed their classrooms to collect data about their teaching practices. A coding system was developed to determine the degree to which teachers (a) emphasized student effort, (b) encouraged students to focus on learning, (c) emphasized performance 
goals such as getting good grades, (d) encouraged autonomy, and (e) emphasized speed. Other coded practices included the frequency with which teachers made comparisons between students, the kind of affect they displayed, the level of their enthusiasm, and whether they fostered a threatening or risk-supportive environment.

The students completed a survey to assess their motivations early in the school year and again following the completion of a unit on fractions. Stipek et al. (1998) collected additional data by observing the behavior of selected students on the videotapes. To measure achievement on the fractions unit the students completed a paper-and-pencil test consisting of 7 procedurally-oriented items and 13 conceptuallyoriented items. With these data, Stipek and her colleagues were able to delineate between mastery-oriented and performance-oriented classrooms, and compare students' behaviors and achievement in these classrooms. They found the more teachers demonstrated positive affect and mastery orientation, the more students reported help seeking, learning for understanding, and positive emotions. Mastery-oriented teacher practices also appeared to have positive effects on students' learning. On the achievement test, students in these classrooms made substantially greater gains on the items that focused on conceptual understanding. However, there was no significant correlation between teacher practices and the gains students made on the procedural items.

Motivational researchers believe that mathematics classes are rich settings to examine goals (Turner et al., 1998). They argue that the culture of traditional mathematics classes values performance, thus making students' personal goal orientations more salient. In other words, Turner and her fellow researchers believe that 
traditional mathematics classes have a performance classroom goal structure that encourages the adoption of personal performance goals by students.

Harrackiewicz et al. (2000) claimed that personal performance goals are general, and not discipline specific, because these goals focus on a desire to outperform others rather than concentrating on the content of the course. Therefore, students with performance goals were likely to exhibit those goals in all of their classes. However, Anderman and others (1999) found that students were more performance-oriented in their mathematics classes than in their English classes. Possibly the culture of traditional mathematics classes encouraged and enhanced the demonstration of the performance goals a student has generally adopted.

Nicholls and others (1990) maintained that teaching practices have considerable bearing on the personal goal orientations of students. According to Turner and others (1998), mathematics teachers should realize that teaching practices with an emphasis on speed, normative scores, and correct execution of algorithms increase student concern about comparative ability. They asserted that teachers need to spend as much time supporting student thinking and reasoning as they do in encouraging performance. These recommendations are consistent with the establishment of a classroom mastery goal structure and the practices recommended by $\operatorname{NCTM}(1989,1991,2000)$.

Harrackiewicz and others (2000) asserted that a personal mastery goal orientation is more specific to the discipline. In other words, the adoption of personal mastery goals will vary depending on the subject under study. Since mastery goals focus on the task and not on ability, the task is more significant to the adoption of these goals. Therefore, 
the establishment of a classroom mastery goal structure in mathematics classes may be requisite to the adoption of personal mastery goals by students in mathematics.

The review of literature to this point has concentrated on classroom goal structures. Researchers found that classroom goal structures established by teachers influenced students' personal achievement goals (Harrackiewicz et al., 2000; Nicholls et al., 1990; Turner, 1998). In the following section, attention will turn to the personal achievement goals that students adopt.

Research on Personal Goal Orientation

Effect of the learning climate.

Nicholls et al. (1990) studied the personal goal orientations of second-grade students in six different classrooms. In one of the classrooms, the teacher instructed students in mathematics throughout the year using a problem-centered mathematics program consistent with constructivist views. Problem solving is a focus in mathematics education reform, and NCTM has endorsed the importance of this activity by listing it as one of the five process standards through which students should acquire knowledge (NCTM, 2000). Nicholls and his colleagues designed a questionnaire especially for young students to measure achievement goals in mathematics. They administered this questionnaire to all six classes at the end of the school year. Their results suggested that a problem-solving approach encouraged a mastery-orientation in students, and that teaching practices have substantial effect over the type of goals students adopt. Cobb and others (1991) conducted a similar study using a larger sample of second-grade students with comparable results. 
Wood and Sellers (1997) also studied the effects of using a problem-centered curriculum on students' beliefs and motivations. The participants of their project were part of a larger longitudinal project that also examined the effects of this curriculum on achievement. One group of students received instruction based on a problem-centered curriculum for one year in second grade, another group for two years in second and third grade, and a third group received traditional textbook instruction in both second and third grade. All students received traditional instruction in the fourth grade. The students completed questionnaires that measured their beliefs and motivations at the end of the third grade and again at the end of the fourth grade. The analysis of these data indicated that students who had received the problem-centered curriculum were not as likely to be motivated by competition with others as the students who received a traditional textbook curriculum (i.e. less performance oriented). In addition, the results indicated that a problem-centered curriculum produced students who were more interested in finding their own ways to solve problems (i.e. more mastery oriented). These results support the findings of Nicholls et al. (1990); however, after returning to traditional instruction for a year, this orientation to mastery goals diminished.

Concerned with the decline in motivation as students moved from the elementary to the middle school levels, Anderman et al. (1999) conducted a longitudinal study to investigate the achievement goals of students through this transition. Middle school students completed surveys measuring their achievement goals at the end of fifth, sixth, and seventh grades. During the fifth grade they attended various elementary schools in the same district and transitioned to two district middle schools in the sixth grade. One of these schools, Beta Middle School, was characterized as moving toward a mastery- 
oriented learning environment, and the other school, Alpha Middle School, represented a more traditional performance-oriented learning environment. Therefore, the elementary students were transitioned into two different motivational climates. Results indicated that mastery goals decreased significantly for all students in grade six, and students' perceptions of a mastery classroom goal structure also decreased significantly. Overall, there were no significant differences in performance goals; however, students who attended Alpha Middle School reported significantly higher levels of performance goals than those who attended Beta Middle School. Also, students' perceptions of a performance classroom goal structure increased significantly for students in grade six. These results suggest that as students transition to middle schools they become more performance-oriented and less mastery-oriented.

Di Cintio (1996) had results similar to Anderman et al. (1999) in a study of differences between the learning environments of elementary and middle schools. His data revealed that fifth-grade students had significantly higher mastery-orientation than did sixth- and seventh-grade students, however his data indicated no significant differences in performance goals. The findings of Anderman et al. (1999) and Di Cintio (1996) support the results of other research that suggests the learning environment affects the achievement goals of students (Cobb et al., 1991; Nicholls et al., 1990; Wood \& Sellers, 1997).

\section{Relation to academic performance.}

The results of research on the relationship of personal achievement goal orientation and academic performance are mixed. Urdan and others (1997) found a positive relationship between grade point average and mastery goals. Their subjects were 
eighth-grade students and the research was situated in a mathematics classroom.

Conversely, Elliot and Church (1997) found that mastery goals had no affect on grades with undergraduate psychology students. Barron and Harackiewicz (2001) conducted an experimental study that also used undergraduate psychology students as subjects. They measured the students' goals using a self-report survey and then gave them instruction in a mental strategy for multiplication. After this instruction, they tested the students on their use of the strategy. Results revealed that mastery goals had no effect on their test score, but that performance goals did predict their performance on the test.

Barron and Harrackiewicz (2001) sought to duplicate these results with another study where goals were assigned, rather than measured through self-report means. For example, to assign the subjects a performance goal, the researchers told subjects that the purpose of the session was to evaluate how well students could perform mathematics problems using a new way of doing mathematics. The researchers told the subjects that performance was the goal and that they should focus on how using the technique could help them solve more mathematics problems than other students. To assign a mastery goal, students were told that the purpose was to learn a new way of doing mathematics, that their goal should be to learn the technique, and that they should focus on how it could improve their mathematics skills. Barron and Harrackiewicz were disappointed that these results did not confirm the outcomes of their first study. There was no relationship between performance on the test and assigned goals of either type.

However, Barron and others (2001) obtained results indicating that performance goals predicted academic performance. In this study, the researchers followed 471 students through their entire college career. They measured students' mastery and 
performance goals early in their first semester in an introductory psychology course. Five years later they examined the students' academic records to determine an overall grade point average for subsequent semesters. Results revealed that performance goals positively predicted academic performance in both the initial psychology class and over the long term measured by the subsequent overall grade point average.

\section{Relation to students' attitudes, beliefs, and learning behaviors}

Much of the research on personal goal orientations focused on the effect that the adoption of specific goals has on an assortment of variables related to students' attitudes, beliefs, and learning behaviors (Archer, 1994; Barron \& Harackiewicz, 2001; Barron et al., 2001; Elliot \& Church, 1997; Meece et al., 1988; Middleton \& Midgley, 1997; Midgley et al., 1996; Ryan \& Pintrich, 1997; Seifert, 1995; Urdan et al., 1997). Most of these researchers conducted studies in the context of public schools (Meece et al., 1988; Middleton \& Midgley, 1997; Midgley, Arunkumar, \& Urdan, 1996; Ryan \& Pintrich, 1997; Seifert, 1995; Urdan et al., 1997); however, several focused on the goal orientations of college students (Archer, 1994; Barron \& Harackiewicz, 2001; Barron et al., 2001; Elliot \& Church, 1997). A review of the findings of these researchers generates a profile of students characterized by specific goal adoption.

In the public school arena, Nicholls et al. (1990) found that, as early as second grade, students with high levels of mastery goals believed that success depended on effort, collaboration, and sense making. By the $5^{\text {th }}$ grade, they also perceived themselves to be competent (Meece et al., 1988). According to Seifert (1995) elementary students who had feelings of competency were likely to adopt mastery goals, and those who adopted mastery goals possessed confidence in their ability to do the work. Mastery- 
oriented elementary students had higher levels of self-efficacy and showed less anxiety related to their classes (Urdan et al., 1997). Mastery-oriented elementary and middle grades students demonstrated a variety of facilitative learning strategies and behaviors. They viewed seeking help as beneficial and were less likely to avoid seeking help when they need it (Middleton \& Midgley, 1997; Ryan \& Pintrich, 1997). They also reported more active cognitive engagement (Midgley et al., 1996) and greater use of self-regulated learning strategies (Middleton \& Midgley, 1997). Mastery-oriented middle grades students indicated higher levels of academic-efficacy (Middleton \& Midgley, 1997), and were less likely to report "feeling dumb" (Ryan \& Pintrich, 1997, p. 335).

At the college level, Archer (1994) reported that students with mastery goals described more positive approaches to learning. This positive attitude included enjoyment, willingness to take similar courses in the future, and perceived relevance of the course. Archer also found that these students reported greater use of metacognitive strategies. Mastery-oriented college students reported higher interest in the subject content (Barron \& Harackiewicz, 2001; Barron et al., 2001) and had higher levels of intrinsic motivation, defined to be the enjoyment of and interest in an activity for its own sake (Elliot \& Church, 1997). Elliot and Church also found that, like elementary students, college students with a mastery orientation perceived themselves to be competent.

Research on performance-oriented students is not as focused. According to Nicholls et al. (1990), second-grade students who acquired performance goals believed success depended on exceptional ability and attempts to outshine one's peers. Seifert (1995) reported that performance-oriented elementary students had a sense of belonging 
which he defined as a need to feel important to teachers and classmates. These students described higher levels of test anxiety (Middleton \& Midgley, 1997) and reported more experiences of negative emotion such as feeling "frustrated and stupid" (Seifert, 1995, p. 549).

Researchers found that performance-oriented middle school students adopt some maladaptive learning strategies and behaviors (Ryan \& Pintrich, 1997; Midgley et al., 1996). For example, these students were more likely to avoid seeking help when needed because they believed that seeking help resulted in unfavorable judgments from others (Ryan \& Pintrich, 1997). In addition, Midgley et al. (1996) reported that students with performance goals were more likely to participate in self-handicapping strategies. Midgley and her colleagues defined self-handicapping strategies as an individual's attempts to manipulate others' perceptions of the reasons for particular performance outcomes. Procrastination is an example of a self-handicapping strategy. If a student puts off studying until the last minute, then a poor test performance can be blamed on limited studying instead of low ability. If the test performance is good, then the student looks even more capable precisely because a good performance occurred despite little studying. This win-win situation protects self-worth but is not conducive to learning. In college students, Archer (1994) found that the adoption of performance goals led to a preoccupation with demonstrating competence or avoiding the demonstration of a perceived lack of competence. She also found that performance goals correlated positively with a positive approach to learning (i.e. enjoyment and interest), and with metacognitive strategies. However, Archer pointed out that these correlations were 
weaker that the ones between mastery goals and both a positive approach to learning and metacognitive strategies.

\section{Research using the three-goal model.}

Urdan et al. (1997) proposed that the weaker relationships between performance goals and other variables found by some researchers may be due to a lack of separation between the two dimensions of performance goals. The initial model by Dweck and Elliott (1983) for goal orientation theory included three types of goals: (a) a mastery goal focused on the development of competence; (b) a performance goal aimed at the development of favorable judgments of competence; and (c) a performance goal directed at avoiding unfavorable judgments of competence. The first two goals were categorized as approach orientations and the third was designated an avoidance orientation.

According to Elliot and Harackiewicz (1996), the idea of separate approach and avoidance performance goal orientations received little attention by researchers and was eventually overlooked. They conducted two experimental studies that investigated the usefulness of separate approach/avoidant performance goals for predicting intrinsic motivation. They predicted that the negative affect of performance goals on intrinsic motivation would only occur in the performance-avoid category.

In the first experiment Elliot and Harackiewicz (1996) assigned undergraduate psychology students either a mastery goal, a performance-approach goal, or a performance-avoidance goal and asked them to solve some puzzles. Intrinsic motivation was measured by recording the length of time the students chose to work on the puzzles during a "free time" period and also by self-report instrument with three items designed 
to measure their enjoyment of solving the puzzles. Task involvement was also measured with the use of a self-report instrument.

The second experiment was similar except that the goal assignment process was more subtle. For example, to assign a performance-approach goal in the first experiment, Elliot and Harackiewicz (1996) told the subjects that most students at their university did quite well but some students stood out in their ability to solve the puzzles. The researchers informed the subjects that this was their opportunity to demonstrate that they were a good puzzle solver. In the second experiment, students assigned to a performance-approach goal were simply told that solving more puzzles than a majority of the university students would demonstrate that they had good puzzle solving ability.

Results from both experiments were essentially the same. Students assigned to performance-avoidance goals demonstrated less intrinsic motivation than students in either the mastery or performance-approach categories. Additionally, the mastery and performance-approach students exhibited the same level of instrinsic motivation, making the adoption of either of these approach goals favorable to learning. Elliot and Harackiewicz (1996) also determined that task involvement acted as a mediator of the detrimental effects of performance-avoidance goals on intrinsic motivation. Students with performance-avoidance goals reported less task involvement than students assigned to either of the other two goal categories. These results substantiate the value of distinguishing between the approach and avoidant components of performance goals.

Elliot and Church (1997) conducted a study to test the mastery/performance approach-avoidance model in a college psychology classroom. They investigated the relationship between these goals and a host of other variables including intrinsic 
motivation, competence expectancy, fear of failure, and graded performance. Final course grades determined the measure for graded performance and all other variables were evaluated with self-report instruments. Achievement motivation was also measured using a subscale of Jackson's (1974) Personality Research Form containing items such as "I enjoy difficult work", and "I often set goals that are difficult to reach."

Results supported the findings of Elliot and Harrackiewicz (1996). A factor analysis upheld the trichotomous framework of three independent goals, and results showed that performance-approach and performance-avoid goals are separate orientations with different effects. Students with mastery goals had high achievement motivation, high competency expectancies, and enhanced intrinsic motivation. Mastery goals were not linked to fear of failure and they had no effect on graded performance. Performanceapproach goals were linked to high achievement motivation, high competency expectancies and fear of failure. These goals had a positive correlation with grades, with no effect on intrinsic motivation. Students with performance-avoid goals had low competency expectancies, poor intrinsic motivation, and lower grades. These goals were also linked to fear of failure. The findings clearly portray different profiles for students with performance-approach or performance-avoid goals (Elliot \& Church, 1997).

Middleton and Midgley (1997) conducted a study testing the three-goal model using sixth-grade mathematics students as subjects. They found that mastery goals positively predicted academic efficacy and negatively predicted avoiding help seeking. Performance-approach goals were not a significant predictor of either of those behaviors, but they were positive predictors of test anxiety. Performance-avoidance goals negatively predicted academic efficacy and positively predicted both avoidance of help seeking and 
test anxiety. These deleterious effects of adopting performance-avoidance goals were similar to those found by other researchers (Elliot \& Church, 1997; Elliot \& Harackiewicz, 1996), but Middleton and Midgley did not find that adopting performanceapproach goals was favorable to learning. They maintained the distinction between mastery and performance goals is the most important determiner of achievement behavior. However, they also noted that performance-avoidance goals were the strongest predictor of avoiding help seeking making these goals a significant factor in classroom performance; therefore, additional research should be conducted using different age groups and other disciplines.

Kaplan and Midgley (1997) investigated whether perceived competence moderated the relationship between goal orientation and learning strategies. The subjects were seventh-grade middle school students in English and mathematics classes. All variables were measured with self-report surveys. Contrary to results of other researchers (Dweck \& Leggett, 1988), they found that a performance goal orientation was not related to reports of adaptive learning strategies and was positively related to reports of maladaptive learning strategies regardless of the level of perceived competence. To explain this result, Kaplan and Midgley suggested that when performance-oriented students with high perceived competence reflected on their behavior in order to complete a survey, they may consider situations that would make their behavior similar to students with low perceived competence. For example, if there were risks for making mistakes in public, then the behavior of performance-oriented students may be similar despite their level of perceived competence. This argument seemed to suggest that performance goals include elements of both approach and avoidance tendencies. 
Another surprising result was that perceived competence did moderate the relationship of mastery goals and learning strategies (Kaplan \& Midgley, 1997). Mastery-oriented students with high perceived competence reported more adaptive and fewer maladaptive learning strategies. To account for this phenomenon, Kaplan and Midgley pointed out that the research was situated in two traditional middle schools that typically maintain a performance-oriented learning climate. The emphasis the environment placed on performance may have made perceived competence more important even to students focused on mastery goals. The researchers also suggested that students may actually pursue more than one goal. The analysis techniques they used did not distinguish between students who had high levels of both mastery and performance goals and students who had high levels of mastery goals and low levels of performance goals. Kaplan and Midgley argued that level of perceived competence may still influence mastery-oriented students if they also retained high levels of performance goals. They pointed out the lack of discussion about the effects perceived ability may have on the behavior of students who pursue multiple goals.

Research on the pursuit of multiple goals.

Most researchers treat achievement goals as dichotomous objectives, but results of some studies have lent support to the idea that students may and should pursue more than one type of goal simultaneously (Barron \& Harackiewicz, 2001; Harackiewicz et al., 2000). For example, Barron and Harackiewicz (2001) asserted that because performance goals positively related to performance and mastery goals positively related to interest, that both types of goals are desirable. On the other hand, Urdan et al. (1997) argued that performance goals were not helpful if strong mastery goals were present. Still others 
maintained that the ideal profile is for students to adopt mastery and performanceapproach goals, coupled with the absence of performance-avoid goals (Elliot \& Church, 1997). Some researchers have analyzed how the pattern of goals or relationship between goals affects outcome beliefs and behaviors (Archer, 1994; Meece \& Holt, 1993; Ng, 1999; Turner et al., 1998).

In 1994, Archer hypothesized that students' level of mastery goals rather than their level of performance goals would predict a positive approach to learning and reported use of metacognitive strategies. The subjects were three groups of first-year college students enrolled in psychology courses. The students completed self-report instruments to measure their mastery, performance, and academic alienation goals. Archer defined the academic alienation goal as a goal to complete academic work with as little effort as possible. This is similar to the work-avoidant goal described by Meece et al. (1988). The students also completed surveys to measure their learning strategies, positive approach to learning, preference for difficult tasks, causal attributions, and perception of ability.

Archer (1994) used a median split method to create four groups of students based on their level of mastery and performance goals: (a) high mastery-high performance, (b) high mastery-low performance, (c) low mastery-high performance, and (d) low masterylow performance. She performed a MANOVA as well as individual analyses of variance on the three dependent variables: learning strategies, positive approach to learning, and preference for difficult tasks. Although significant relationships were found between performance goals and learning strategies, and between performance goals and a positive approach to learning, Archer claimed the most central result from these analyses was a 
highly significant main effect for mastery goals. She pointed out that the positive correlations found with performance goals were weaker than the positive correlations found with students who had high levels of mastery goals. She also highlighted differences in performance-oriented students with high perceived ability and those with low perceived ability as a rationale for these relationships. This argument supported the performance approach-avoidant model studied by other researchers (Elliot \& Church, 1997; Elliot \& Harackiewicz, 1996; Middleton \& Midgley, 1997).

Suggesting that the median split method of studying multiple goals created an arbitrary division, $\mathrm{Ng}$ (1999) used a goal web theory to guide his research. In this theory, performance and mastery goals are just two of a number of goals that a student may possess; however, these are the goals most directly linked with learning tasks. Other goals may provide additional support with respect to learning. For example, social goals, characterized as "meeting family expectations" or "pleasing the teacher" may influence the affects of mastery or performance goals. Ng described a goal web as "a cognitive schema or a hierarchical mental structure consisting of a network of causally-linked learning goals held by individual learners" (p. 5). In a web, the effect of a particular goal may change as a result of causal links to other goals. For example, if a student claims to like mathematics, which is indicative of a mastery goal, but likes mathematics because she is good at it, an indication of performance goals, then the positive affect of the mastery goal abates because of the student's concern over performance.

Ng's (1999) study was qualitative. The subjects were thirteen high school students selected based on their achievement scores and self-schema construct scores taken from a survey administered in an earlier study. These participants were students at 
three different high schools in Brisbane, Australia. The researcher conducted a series of in-depth interviews with each of the subjects during their lunch break or during their mathematics lesson.

The results of this study revealed underlying differences between the goal webs of high achievers and low achievers ( $\mathrm{Ng}, 1999)$. While all students gave a variety of responses indicative of performance goals, only high achieving students frequently invoked mastery goals as a reason for studying mathematics. Ng claimed that high achieving students had more complex goal webs with stronger links and more goal diversity. He maintained that the data from this study supported the notion of multiple goals and arguedthat future research should investigate the combined effects of goals.

Turner et al. (1998) used a cluster analysis procedure to examine the goal patterns and negative affect of fifth and sixth grade students in their mathematics class. The analysis included seven variables: mastery goals, performance goals, negative affect after failure, deep strategy use, self-efficacy, action after failure, and preference for difficulty. What emerged were four distinct patterns of motivation, affect and behavior:

1) Learning Oriented - This group had the highest mastery goals, lowest performance goals, lowest negative affect after failure, high self-efficacy, and more positive learning behaviors after failure.

2) Success Oriented - This group had the second highest level of performance goals, high self-efficacy, but were not as committed to learning.

3) Uncommitted - These students had mastery and performance goals that were both weak, and demonstrated lower self-efficacy and a commitment to learning similar to the success oriented group. 
4) Avoidant - This group was nearly a mirror image of the Learning Oriented group. They had the lowest mastery goals, highest performance goals, highest negative affect after failure, lowest self-efficacy, and more negative learning behaviors after failure.

The analysis showed that increased negative patterns of affect occur in two cases: (a) when mastery goals are lower and performance goals are higher, and (b) when both goals are relatively equal and low. This examination of goal patterns and affect made this research original.

Meece and Holt (1993) reanalyzed the data from a previous study (Meece et al., 1988) to examine how differences in the strengths of mastery, performance (ego), and work-avoidant goals affected fifth and sixth grade students' academic performance in science. They used a cluster analysis procedure to combine students into groups based on their pattern of goals. The results of the procedure identified three separate clusters: (a) high mastery, (b) combined mastery-ego, and (c) low mastery-ego.

Students in Cluster 1, the high mastery cluster, had mastery scores that were significantly higher than their performance and work-avoidant scores. There were no correlations among the three types of goals in this group. The mastery scores in this cluster were also significantly higher than the mastery goals in the other two clusters.

Cluster 2, the combined mastery-ego cluster, included students who were equally strong in mastery and performance goals. There was also a positive correlation between mastery and performance goals. In addition, both mastery and performance goals correlated negatively with work-avoidant goals. Students in this group had significantly higher performance goals than those in the other clusters. 
In the low mastery-ego cluster, there were no significant differences between the three types of goals. There was no correlation between mastery and performance goals in this group, but there was a positive correlation between performance and work-avoidant goals. Students in this cluster reported significantly higher work-avoidant goals than students in the other two clusters.

The researchers then analyzed the clusters for their affect on engagement, effort, teacher expectations, science grades, and achievement test scores (Meece \& Holt, 1993). Cluster 1, the high mastery group, scored significantly higher than the other two clusters on effort, teacher expectations, science grades and achievement scores. Cluster 2, the combined mastery-performance group, and Cluster 3, the low mastery group, had similar scores on these variables. Since Cluster 2 did not score as well as Cluster 1 on these performance variables, these findings supported the assertion of Urdan and others (1997) that performance goals are not at all helpful if mastery goals are strong. Clusters 1 and 2 scored equally high on active engagement. Cluster 3 scored the highest on superficial engagement. Further analysis indicated gender and teacher differences in the clusters. Cluster 1 contained many more females than males, and the reverse was true for Cluster 3. Many of the students in Cluster 1 were associated with a particular teacher, and a large number of students in Cluster 3 were associated with two specific teachers. The results of this study supported the conclusions of other researchers that fostering a mastery orientation in students is important for learning to occur (Ames, 1992; Ames \& Archer, 1988; Meece et al., 1988).

\section{Summary}


Thus far, I have reviewed literature regarding research on achievement motivation. Some of the more salient points from this research are as follows:

1. The learning environment or perceived classroom goal orientation affects the learning behaviors and achievement goals that students adopt (e. g. Ames \& Archer, 1988; Kumar, 2004; Nicholls et al., 1990; Patrick et al., 2001; Turner et al., 2002; Urdan et al., 1998; Wood \& Sellers, 1997).

2. Mathematics classes are traditionally performance-oriented and therefore encourage the adoption of performance goals (Turner et al., 2002; Anderman et al., 1999).

3. Students with mastery goals exhibit more adaptive learning behaviors and more confidence (e. g. Ames \& Archer, 1988; Elliot \& Church, 1997; Meece et al., 1988; Middleton \& Midgley, 1997; Seifert, 1995; Urdan et al., 1997).

4. Results of the affects of performance goals are mixed (e. g. Archer, 1994; Middleton \& Midgley, 1997; Seifert, 1995). Some researchers argued that these mixed results occur because there are two different types of performance goals, performanceapproach and performance-avoidance, that should be analyzed independently (e. g. Elliott \& Harackiewicz, 1996; Urdan et al., 1997).

5. Several researchers maintained that students pursue multiple goals and that future research should examine the patterns of goals rather than the adoption of one type of goal over the other (e. g. Barron \& Harackiewicz, 2001; Harackiewicz et al., 2000).

In the section that follows, I will shift the focus of this literature review to studies concerning student and teacher attitudes toward mathematics. 


\section{Attitude}

Attitudes are described as being learned tendencies to respond in a generally favorable or unfavorable manner toward some object. Research on attitude may focus on one or more of four conceptual distinctions of attitude: (a) affect, (b) cognition, (c) conation, and (d) behavior (Fishbein \& Ajzen, 1975). Affect refers to feelings toward some object, while cognition indicates beliefs about the object. Conation refers to a person's intentions to perform certain behaviors, and the behavior category represents the actual observed overt acts. This research focuses on the concept of cognition or beliefs. Fishbein and Ajzen contended that a person's attitude is a function of their beliefs. Their conceptual framework employed beliefs as the fundamental building blocks.

Beliefs represent the information one has accumulated through past experience with the object (Fishbein \& Ajzen, 1975). For education, this means that attitude toward school is a result of the evaluation of past experiences with schooling. Similarly, for mathematics or any particular subject, attitude toward the subject is the result of evaluation of past experiences with the subject.

\section{Student Attitudes}

McMillan (1976) reviewed 124 dissertations completed between 1969 and 1975 concerned with factors that affected students' attitudes toward various school subjects. His analysis of these studies revealed that noncurricular variables have at least as much impact on attitude as curriculum-related variables. A large percentage of significant findings came from studies relating attitude to teacher behavior, student self-concept, background, parents, and previous attitudes. McMillan pointed out that numerous research reports from journals support his findings (Phillips, 1973; Walberg, 1969). 
These studies indicated that teacher attitudes and classroom climate are among the most significant factors related to attitude formation.

McMillan's (1976) results support the theory that attitudes are based on an evaluation of past experiences with the object. In education, these experiences would include the classroom climate established by teachers for a particular subject. Numerous studies have since indicated that a decline in positive attitudes toward mathematics is at least partially due to the classroom climate and lack of teacher support (Ames, 1992; Ames \& Archer, 1988; Gottfried, 1985; Lepper, 1988; Meece et al., 1990; Middleton, 1992; Midgley et al., 1989).

Why are attitudes important? The desired outcome is student achievement, and a strong relationship between attitude and achievement has long been assumed (Ma \& Kishor, 1997). However, a review of the research in this area uncovers results that are not conclusive. Aiken (1972b) and Boswell and Katz (1980) concluded that attitude was positively related to achievement, but other researchers determined that attitude was not a strong correlate of achievement (Robinson, 1975; Vachon, 1984). However, one pertinent result of this line of research is the finding that the attitude-achievement relationship is stronger for females than for males (Aiken, 1976; Fennema \& Sherman, 1976a).

Ma and Kishor (1997) conducted a meta analysis of the research on attitude and achievement in mathematics in an attempt to make sense of the inconsistent findings. They wanted to establish the existence of the relationship, explore factors that affect the relationship, and determine the magnitude of the causal relationship. Ma and Kishor searched leading journals in education, especially mathematics education, to find a 
sample of studies meeting a list of pre-established criteria. This search resulted in a sample of 113 studies that dealt primarily with the relationship between attitude and achievement in mathematics. The results of their meta-analysis established that a relationship exists, but it is not strong. They also concluded that gender had no affect on the relationship. The causal relationship, achievement causes attitude, was not significant, but the causal relationship, attitude causes achievement, was significant. However, since the magnitude was small, they claimed the relationship was not useful for practical application. Then again, the researchers pointed out that this relationship got stronger as students got older, so that by the time students reach the high school level the relationship might be practically meaningful. If this is the case, then attitude toward mathematics might play a significant role in the mathematics achievement of preservice elementary teachers.

A belief about self-confidence in learning mathematics is an attitude variable that has received considerable attention in past research on student attitudes toward mathematics (Kloosterman, 1988). Students who lack confidence in their skills are not as likely to pursue activities that require those skills, and they are less likely to persist when faced with difficulties (Pajares \& Miller, 1994). Aiken (1972b) determined that mathematics attitude was positively related to self-confidence in eighth grade students. Several studies found that females exude less confidence than males in the mathematics classroom (Fennema \& Sherman, 1976a; 1978; Iben, 1991) and more negative attitudes toward mathematics (Boswell \& Katz, 1980). A recent study by McGraw, Lubienski, and Strutchens (2006) determined that female attitudes continue to be more negative than males, although both genders now report similar levels of understanding. Additionally, 
McGraw and others found that attitude and self-confidence correlated positively with achievement in both males and females. These findings indicate that lower selfconfidence and attitudes that are more negative may result in lower levels of achievement for females than for males.

In a three-year longitudinal study, several researchers analyzed the beliefs and attitudes of students in grades one through six in an attempt to determine which beliefs change with age (Kloosterman \& Cougan, 1994; Kloosterman et al., 1996). They found no relationship between confidence and achievement in mathematics among first grade students. In second grade, however, high-achieving students had more confidence. The researchers concluded that by the end of the second grade students had an accurate perception of their ability in mathematics.

This finding highlights the importance of helping intermediate elementary students to focus on effort and believe that effort is worth their while. If they attribute success to ability and have a low perception of their own ability, then their motivation will suffer. Teachers in these grades need to establish a mastery-oriented classroom climate to encourage a mastery orientation in these students. Archer (1994) reported that while perception of one's ability does affect attitude, a mastery goal orientation provides an even greater role in attitude development. Additionally, Ames and Archer (1988) concluded that attitudes were a function of students' perception of a mastery classroom goal orientation.

Kloosterman (1988) attempted to explain self-confidence through motivational variables. One of the motivational variables he considered was attributional style. He suggested that a positive attributional style score was indicative of a mastery goal 
orientation. The subjects in this study were 489 seventh grade students from three small cities in Indiana. Kloosterman found that attributional style was the strongest predictor of confidence. In addition, all of the motivational variables had a significant correlation with self-confidence and with each other. In a later study, Stipek and others (1998) had similar results with research on self-confidence. Their findings indicated that students who self-reported high perceptions of confidence in their mathematics abilities were also more focused on learning and mastery.

A belief about the usefulness of mathematics is another important attitudinal variable to consider. Since mathematics is not easy for most people to learn, it is reasonable to question making the effort if one does not believe in its utility (Fennema \& Sherman, 1976a). Fennema and Sherman (1978) reported that across grades six through eleven, males perceived mathematics as being more useful than females. A later study separated the mathematics usefulness scale into "usefulness for males" and "usefulness for females" (Boswell \& Katz, 1980). Results from this study revealed that even males perceived mathematics to be less useful for females than for themselves. Additionally, as females matured, they saw less usefulness of mathematics for themselves while maintaining the same perception of usefulness for males.

Kloosterman and Cougan (1994) and Kloosterman and others (1996) explored elementary students' beliefs about the usefulness of mathematics. They concluded that primary students did not have a sincere belief that mathematics was useful. When questioned about why mathematics was important, most of these students responded with comments about needing it to pass to the next grade. Responses from upper elementary students were not much better. While some older students claimed to believe 
mathematics was a useful subject, very few could provide any substantial examples of real world applications.

Other researchers have investigated the correlation between beliefs about usefulness and achievement in mathematics. Iben (1991) and Midgley and others (1989) found a positive relationship between these variables. Midgley and her colleagues studied the relationship between usefulness, achievement and teacher support as students transitioned from elementary to middle school. They defined teacher support as students' perceptions of the teacher as being caring, friendly, and fair. The findings of this study strongly supported a positive relationship between the perceived level of teacher support and student's beliefs about the usefulness of mathematics. When students transitioned from an elementary school, where they perceived a high level of teacher support, to a middle school, where they perceived a lower level of teacher support, their beliefs about the usefulness of mathematics dropped sharply. Furthermore, for low achieving students, this type of transition resulted in a steeper decline in beliefs about usefulness than for high achieving students. It is interesting to note that the transition from elementary to middle school is also often a move from a mastery-oriented classroom climate to a performance-oriented classroom climate (Anderman, 1999; DiCintio, 1996).

Beliefs are often culturally-based and passed down from generation to generation. This study will be conducted in a rural region of Appalachia with a culture that is unique to other parts of the county and other rural areas (Bush, 2003). The usefulness attitude variable for mathematics may be particularly salient for students in this region. According to many researchers, rural Appalachia struggles with believing in the value of a mathematics education (Bush, 2003; DeYoung, 2003; Howley, 2003). According to 
DeYoung, students in some rural Appalachian schools “...attributed lack of math achievement (if any) to their own lack of interest..." (p. 68). Bush (2003) notes that while community members in this region may value literacy, they may view the mathematics curricula taught in schools as disconnected from the community.

Another attitude construct relevant to the present research is the notion that mathematics is a subject best pursued by males. Fenema and Sherman (1976b) described this attitude variable as the belief that mathematics is a male domain. Several studies found that lower-achieving females are more likely to consider mathematics to be a subject for males (Boswell \& Katz, 1980; Iben, 1991; Fennema \& Sherman, 1976a, 1978). Fennema and Sherman argued that females are not as likely to excel in an area they perceive to be masculine. Since most elementary teachers are female, this belief would present a barrier to learning mathematics content themselves and in encouraging their future female students to excel in this subject as well. Furthermore, teachers who hold this belief may be predisposed to use teaching strategies in the mathematics classroom that favor boys. This teaching tends to be traditional performance-oriented instruction that encourages the adoption of performance goals (Anderman et al., 1999; Nicholls et al., 1990).

There have been some mixed results in the research on the mathematics as a male domain variable. Fennema and Sherman (1976a, 1978) reported that as females moved into high school they were less likely to consider mathematics to be a male domain. On the other hand, Boswell and Katz (1980) ascertained that older females perceive themselves as less capable than males. However, all of these researchers agreed that 
males considered mathematics to be a male domain even more so than females (Boswell \& Katz, 1980; Fennema \& Sherman, 1976a, 1978).

In summary, this review revealed that attitudes are based on beliefs that develop from prior experiences (Fishbein \& Ajzen, 1975). It is logical to conclude that past encounters with mathematics will include experiences in mathematics classrooms. Several researchers have noted that a decline in positive attitudes toward mathematics is due in part to the classroom climate (Ames, 1992; Ames \& Archer, 1988; Gottfried, 1985; Lepper, 1988; Meece et al., 1990; Middleton, 1992; Midgley et al., 1989). Confidence in learning mathematics, the perception that mathematics is useful, and the notion that mathematics is a male domain are some of the more salient beliefs underlying attitude toward mathematics that have been scrutinized by other researchers (e.g. Boswell \& Katz, 1980; Fennema \& Sherman, 1976a, 1978; Kloosterman, 1988; Kloosterman et al., 1996). These beliefs are formed in part by the mathematics classroom environment that students experience. Classroom climate is a condition that is to a great extent under the teacher's control. The following section reviews literature concerning teacher and preservice teacher attitudes toward mathematics and the effect of those attitudes on the classroom environment they provide their own students.

\section{Elementary Teacher and Preservice Teacher Attitudes}

Since the overwhelming majority of elementary teachers and preservice teachers are female, it is no surprise to find a high level of negativism toward mathematics among this group. Bulmahn and Young (1982) hypothesized that the kind of person who is attracted to elementary school teaching is "not necessarily the kind who enjoys mathematics" (p. 55). Many elementary teachers have poor backgrounds in mathematics 
as well as negative attitudes (Meyer, 1980). Compounding the problem is their refusal to work toward improvement in this subject area (Meyer, 1980). Bulmahn and Young found that many preservice teachers felt that being proficient in computation was all they needed, and as long as they had a teacher's manual they could adequately teach the subject.

Meyer (1980) argued that the first step in rectifying this problem was to identify the source of these negative attitudes. According to Banks (1964), the attitudes of prospective elementary teachers' own previous teachers are the most significant contributing factors in formulating their own attitudes. Other research has supported this finding (Aiken, 1972a; Ernest, 1976; Karp, 1988, 1991; Meyer, 1980; Nicholls et al., 1990). Teachers impose their own attitudes and beliefs toward mathematics on their own students (Meece et al., 1990; Midgley et al., 1989), and many of these students eventually become teachers themselves. Thus, a detrimental cycle of negative attitudes toward mathematics continues.

Many studies have investigated various intervention strategies targeting preservice teachers in order to break this cycle. In 1980, Tishlor studied 26 junior and senior elementary education majors to see if attitudes toward mathematics could be improved with remedial instruction. Although achievement improved, there was no significant change in attitude.

Gibson, Brewer, Magnier, McDonald and Van Strat (1999) found that mathematics courses for preservice teachers that used more constructivist strategies did improve attitudes. However, their study revealed that attitudes did not change because of 
one course, but the positive change was a trend that became significant by the end of the third course.

Quinn (2001) investigated the effect that a mathematics methods course based on NCTM standards had on the attitude of preservice teachers. They found that although attitudes improved, the mean attitude was still not positive. The improvement was from a negative attitude to one that was more neutral. Quinn concluded that a NCTM Standardsfocused elementary mathematics methods course could get attitudes turned in the right direction, but preservice teachers needed additional intervention. This result is consistent with the findings of Gibson et al.(1999) that a single course was not sufficient to make significant changes in attitude.

Other researchers have lamented the fact that university coursework seems to have little affect on beliefs about mathematics that have been shaped by years of first hand experience as students (Ball et al., 2001; Foss \& Kleinsasser, 1996). As a result teachers continue to enter the field with poor attitudes toward mathematics and these attitudes impact their teaching strategies.

Karp $(1988,1991)$ investigated how teachers' attitudes toward mathematics affected their teaching behaviors and strategies. She discovered salient differences in the methods employed by teachers with positive attitudes and those with negative attitudes. Teachers with negative attitudes used methods that fostered dependency. They centered instruction on memorizing rules, learning algorithms, and finding one right answer. These teachers saw their role as being the center of knowledge, and behaved in a manner that cultivated learned helplessness in their students. These behaviors included asking 
and answering their own questions, limiting student involvement, and completing tasks for some students while expecting others to complete them independently.

Teacher actions such as these lead some students to believe that the teacher has low expectations for them. They begin to think that nothing they do will change the outcome of their performance on a task, and that effort is of no use. They perceive a focus on performance and develop low confidence in their ability. This is characteristic of a classroom that is performance-oriented and fosters a performance goal orientation in students.

On the other hand, Karp $(1988,1991)$ found that teachers with positive attitudes toward mathematics encouraged student initiative and independence. Their instruction attended to why an algorithm worked and developed connections between skills. They encouraged exploration and provided resources for self-instruction rather than taking the role of sole provider of knowledge. These behaviors develop the belief in students that their efforts will have a positive impact on their learning, and fosters a mastery orientation.

Wilkins (2002) also studied attitudes of practicing elementary teachers. He argued that teachers with more positive attitudes might be more able to transfer positive beliefs about mathematics to their students. The subjects for this research were teachers participating in a professional development program focused on implementing reform curricula. One important finding was that teachers with more positive attitudes were more likely to believe in the effectiveness of reform-oriented instruction. However, many who claimed to favor this type of instruction did not report frequent use of these 
strategies in their classrooms. This is consistent with the theory that attitudes lead to intentions, but do not always result in the expected behaviors (Fishbein \& Ajzen, 1975).

This section of this literature review has established that many elementary and preservice elementary teachers have poor attitudes toward mathematics, and these attitudes are difficult to change (Bulmahn \& Young, 1982; Meyer, 1980). However, change is possible using constructivist strategies to teach mathematics over a long period of time (Gibson et al., 1999; Quinn, 2001). Attitudes are important because they affect teaching behaviors and strategies (Karp 1988, 1991; Wilkins, 2002). Teachers with negative attitudes exhibit behaviors and utilize strategies that encourage performance goals in students, while those with positive attitudes are more likely to use a mastery approach to instruction that is in agreement with reform-oriented strategies advocated by NCTM.

\section{Cultural Issues}

Evidence is mounting that sociocultural circumstances have a major impact on student motivation in mathematics (Carr, 1996; Hart \& Allexsaht-Snider, 1996). Families convey societal attitudes about the importance of mathematics and establish standards of performance for students. Hart and Allexsaht-Snider suggested that discontinuities between home and school culture result in student resistance to learning. Maehr and Nicholls (1980) argued that concepts of success and failure are culturally dependent, and results of studies may not be transferable across cultural groups. Therefore, culture is an important consideration in motivation research.

The values embedded in a culture can affect teaching, learning, and curriculum (Bush, 2002; Reyes \& Stanic, 1988). Bush identified four cultural factors that have 
emerged over the past twenty years of research on how cultural differences affect students' achievement in and attitudes toward mathematics. These factors are 1) parent attitudes, 2) teacher attitudes, values, and beliefs, 3) student perceptions and beliefs, and 4) language. These culturally-embedded beliefs and resultant attitudes may significantly influence students' motivation to learn.

Parents of different cultural backgrounds convey their beliefs about achievement to their children (Stevenson et al., 1990). Mothers in China and Japan for example, strongly believe in the value of hard work and effort to achieve success in school, which in turn might contribute to the academic success of Chinese and Japanese children. American mothers, however, tend to believe that ability is the more overriding factor (Stevenson, Lee, \& Stigler, 1986). If parents believe ability is the most important factor for success then children may not be encouraged to work as hard. A focus on ability encourages performance goals.

Watkins, McInerney, Lee, Akande, and Regmi (2002) hypothesized that in Asia, since significant others such as parents and peers greatly influenced a student's definition of success, group and affiliation goals would be more salient than individual goals such as mastery or performance. They looked at the effect of socially-oriented motivational goals on learning strategies across six different cultures. They found that superficial learning strategies were associated with performance goals, while deep learning strategies were associated with mastery goals. However, results did not support their hypothesis. Socially-oriented goals did not appear to affect the learning strategies adopted by students from the different cultures. Watkins and others argued that the concept of social goals 
might need to be defined separately for each society, and that scales used to measure learning strategies should be reexamined.

Another cross-cultural study of motivation looked at the impact of students' selfschema on their mastery versus performance goal orientation and learning strategies in mathematics (Ng \& Renshaw, 2002). An academic self-schema for a specific subject develops as a student generalizes past experiences in learning that subject. When a student develops a positive self-schema towards a subject it becomes part of their selfconception - who they are (e.g. I am someone who is good at math). $\mathrm{Ng}$ and Renshaw maintained that cultural values surrounding academic success affected the development of self-schemas. Their findings revealed a significant influence of self-schema on both motivation and learning engagement among Chinese and Australian students. In both cultures, positive self-schemas had stronger effects on the adoption of mastery goals over performance goals, but the relationship between self-schema and performance goals was much stronger in the Chinese students than in the Australians. Ng and Renshaw attribute this to the competitive nature of the educational system in Hong Kong, claiming these results suggest a significant sociocultural influence.

Midgley et al., (1996) studied and compared the achievement goal orientations and self-handicapping strategies of European American and African American eighthgrade students. They hypothesized that performance-oriented students would be more likely to use self-handicapping strategies such as withdrawing effort in an attempt to protect their image as being able. They also theorized that there would be no relationship between mastery goals and self-handicapping. Self-report instruments were used to collect data for this study. The results showed that self-handicapping was positively 
related to performance goals and that this relationship was significantly stronger in African American students than in European American students. As predicted, there was no relationship between self-handicapping and a mastery orientation. The researchers suggested the possibility that African American students are more adversely affected by a performance-orientation to learning than are European Americans.

Because most motivational studies focus on cognition with no attention to context, Hufton, Elliott and Illushin (2003) chose to concentrate on precisely those social elements that might influence student achievement motivation. These included the impact of significant others, classroom and schooling contexts, and historical, socioeconomic and socio-cultural traditions and changes. They chose three cultures to investigate, not because they were representative of a particular country, but because they demonstrated marked differences in student motivation. The three cultures in this study were Sunderland, in northeast England, St. Petersburg, in Russia, and eastern Kentucky in the United States. Hufton and his colleagues used a qualitative approach involving extensive and numerous interviews with students, teachers, parents, administrators, teacher educators, and student teachers in each of the three cultures.

The findings reported by Hufton and others (2003) were stated as similarities and differences in teachers' opinions across the three cultures. One interesting finding was that teachers in Sunderland and eastern Kentucky perceived community influences as a cause of student lack of motivation; whereas in St. Petersburg this was not a concern. Among the most salient findings was that teachers perceived rewards to be motivating for students. They also believed that grades were effective for motivating younger students but became less effective as students got older. Future employment possibilities and 
personal interest in the content were also among the important motivating factors cited by the teachers in this study. For high achieving students, English and Kentucky teachers thought that rivalry and competition tended to add to motivation. These teacher beliefs suggest an approach to instruction that is performance-oriented.

Hufton and others (2003) also learned that teachers believed subcultural groups affect student motivation. According to teachers, some community influences actually decrease students' motivation to succeed in school. These communities developed ways of life that rely on state welfare, are characterized by unemployment, and do not value education. The teachers believed that schooling may have been an unpleasant experience for the parents, and for these students conflicts arose between loyalty to their family's way of life and their pursuit of education.

Kannapel and DeYoung (1999) pointed out that educators often have goals that contradict the intent of some parents in rural cultures. For example, the goal of a college education often gives rural students no choice but to leave home for extended periods. Furthermore, prescribed curricula designed to serve national goals can separate rural people from the education of their children. Hufton et al. (2003) noted that teachers in eastern Kentucky struggled to motivate many of their students to achieve when instruction focused on a prescribed curriculum such as the state-mandated core content. Kannapel and DeYoung argued that schools should deliver a curriculum that prepares students to live in whatever locale they choose. National reformers and rural educators should collaborate to develop connections between reform curricula and local community goals. 
The literature reviewed in this section reveals that cultural issues are relevant to student motivation and attitudes toward education and mathematics education. The following section focuses on the rural culture.

\section{The Rural Culture and Motivation}

This proposed dissertation is also a rural study. A rural study is more than simply a study carried out in a rural location (Coladarci, 2003). While the rural context is imperative, the study must also connect with rural issues and seek to explain or investigate a phenomenon that manifests itself in the rural lifeworld. In particular, this study will address the issue of mathematics achievement motivation and attitude toward mathematics in the rural culture.

How might the rural lifeworld affect achievement motivation? According to Theobald (1997) one way a rural culture differs from a nonrural culture is in their attachment to place. Attachment to place is a statement about values. Rural people value their land and their community over economic pursuits, often limiting occupational opportunities (DeYoung, 1995a). Performance-oriented efforts to motivate rural students with these values by using statements such as "You need to learn math to get a good job," will not be effective (Nicholls, 1983), especially when these opportunities necessitate leaving home and community. In nonrural areas, where such opportunity is nearby, performance-oriented strategies may be more effective.

Hyde and Kling (2001) described cultures that are individualistic or collectivistic. An individualistic culture holds the rights of the individual to be paramount, while in a collectivistic culture the individual may be expected to place the goals of some group (e.g. family) ahead of individual goals. They claimed that Asian cultures are 
collectivistic and the dominant culture in the United States is individualistic. However, the rural subculture may be collectivistic. Rural researchers claim that rural people possess a communal, rather than individualistic, orientation (Howley, 2003; Theobald, 1997). Reluctance to leave home for college and taking on part time work to help with family expenses are examples of a communal/collectivistic orientation in rural areas. According to Theobald, the emergence of an individualistic culture was the result of a series of historical events that began with St. Augustine's desire that people should turn inward to analyze whether or not their actions are pleasing to God. In today's world, this righteous aim has metamorphosed into an individual's right to pursue the accumulation of material wealth. This individualistic direction is more in line with an urban lifeworld where business and industry are prominent.

This individualistic vs. communal orientation may affect the type of achievement goals that are dominant in a culture. Hyde and Kling (2001) point out that an individualistic society emphasizes performance goals. In collectivistic Asian cultures, such as China and Japan, mothers attribute success to effort (Stevenson et al., 1990), which is indicative of a mastery goal orientation. Other studies have suggested that people in rural cultures are mastery-oriented. For example, Elliott, Hufton, Illushin, and Willis (2001) found that people in the Appalachian region of Eastern Kentucky, a predominantly rural area, largely attributed success in academic endeavors to effort. In a comparative study, Freeman and Anderman (2005) found that rural students indicated a higher level of mastery goals than did urban students. However, neither of these studies used college students as subjects, and the motivational goals were not specific to the mathematics classroom. 
Researchers have documented differing patterns of behavior in rural and nonrural regions regarding motivation to learn (Elliott et al., 1999; Elliott, Hufton, Illushin, \& Lauchlan, 2001; Elliott, Hufton, Illushin, \& Willis, 2001; Freeman \& Anderman, 2005; Hufton et al., 2003). This study seeks to build on that knowledge and expand it to the content specific area of learning mathematics.

\section{The Appalachian Rural Culture}

The rural interest of this researcher lies primarily with rural Appalachia. Therefore, this study will specifically investigate the achievement motivation and attitudes toward mathematics of preservice teachers from rural Appalachia.

The Appalachian region consists of 410 counties in 13 states. Forty-two percent of the 23 million people living in this region reside in a rural area compared to $20 \%$ living in rural areas in the national population. Although education in the region has improved in recent years, the area still lags behind the nation on traditional measures of educational attainment. For example, only $68.4 \%$ of the adults age 25 and over in the Appalachian region are high school graduates, compared to $75.2 \%$ nationally (http://www.arc.gov).

According to Ergood and Kuhre (1983) most characterizations of the culture of Appalachia are restricted to that part of the region referred to as Central Appalachia. Central Appalachia includes eastern Kentucky, east Tennessee, southeastern Ohio and all of West Virginia (Bush, 2003). Reck (1985) points out that the question of whether Appalachia is a distinct ethnic group or subculture has been debated, and Batteau (1983) discusses a variety of attempts that scholars have made to describe the distinctiveness of the Appalachian subculture from the general rural culture. However, while Appalachian 
scholars may not agree on how to define or describe it with regards to similarities and differences to other rural groups, the existence of this subculture is accepted (Ergood \& Kuhre, 1983).

The Appalachian subculture is, naturally, different from the culture in urban areas and other regions of the country, and it is different from the culture of other rural areas as well (Bush, 2003). According to Jones (1983) “...the Appalachian value system that influences attitudes and behavior is different from that which is held by our fellow countrymen..."(p. 125). Therefore, the beliefs and values of this culture could produce motivations and attitudes toward mathematics in preservice teachers from rural Appalachia that are different compared to those from other rural or nonrural areas.

General values and attitudes.

The literature on Appalachian culture documents some unique values and attitudes among the people in this region. Some use a subculture of poverty explanation to describe rural Appalachia. For example, in their religious beliefs many are often described as being fatalistic (Ford, 1983; Jones, 1983). This attitude has been argued to create a passive acceptance of whatever happens; therefore, some claim that many Appalachians often do not seek change to correct the social problems that exist such as poverty and unemployment. They are described by Weller as holding the belief that everything is fine as it is and that change would just make things worse (Weller, 1965). To compensate for their plight their religion emphasizes that rewards will come in the next world. The people of Appalachia also tend to traditionalism, meaning that they are more likely to look to the past rather than the future (Ford, 1983). This combination of 
fatalism and traditionalism creates a climate that is not conducive to change. As Jones (1969) points out, a change in attitudes and values comes more slowly in Appalachia.

Some Appalachian people have also often been characterized as individualistic and self-reliant (Ford, 1983; Jones, 1983). Individualism in this context means a desire for freedom and independence. Mountain people (Appalachians) take pride in doing things for themselves and avoid asking others for help. According to Jones, a desire to be self-reliant is stronger than a desire to get help.

Jones (1983) discussed the deep feelings of loyalty that many Appalachians have toward their kinfolk. They possess a sense of responsibility that extends even to relatives who are more distant such as second and third cousins, nieces, and nephews. They often demonstrate familism, which is a reluctance to live away from kinfolk (Nafziger, 1971), and are therefore very attached to the region where they were raised. DeYoung (1995a, 1995b) found evidence of this attachment to place in his study of an Appalachian rural high school in Braxton County West Virginia. The young people he interviewed expressed their desire to stay close to their families - to the extent that they would rather live on government assistance than look for work elsewhere. The vocational director of the high school in this study explained the reason for this phenomena by expressing that "There is a sort of security in the mountains" (DeYoung, 1995b, p. 182). Appalachians never forget their native place (Jones, 1983).

Other values and attributes suggest that the people of this region may tend to adopt mastery goals. Ford (1983) conducted a survey to investigate the beliefs, values and attitudes of people in the Appalachian region. His survey included households from both rural (49.4\%) and nonrural (50.6\%) areas of Appalachia. Ford found that very few 
Appalachians (7\%) believed that the most important factor for a person to be successful in work was natural ability. The fact that a person likes his or her work, has a good education and training, or demonstrated hard work all ranked above natural ability. Elliott et al. (2001) confirmed this emphasis when they found that many people in the Appalachian region of eastern Kentucky attributed success to effort rather than ability. In addition, Jones (1983) argued that Appalachians are not disheartened by failure, but expect that failures will occur. These attitudes toward success and failure suggest an orientation toward the adoption of mastery goals

However, the Ford (1983) study also revealed that the rural Appalachians were somewhat more likely to attribute success to natural ability than the nonrural Appalachians. This suggests that perhaps the rural Appalachians are less likely to adopt mastery goals than nonrural Appalachians. However, when comparing characteristics of rural and nonrural societies one must pay close attention to how the researcher defines these locales. In the Ford study, the nonrural participants came from metropolitan areas, defined as counties with cities that have a population of 50,000 or more and the surrounding counties, and from other towns and cities with populations of 2500 or greater. The rural participants lived in smaller villages and open country. Different definitions of rural and nonrural could result in a different outcome.

According to Jones (1983) many Appalachians tend to avoid confrontation because they do not want to offend others. They do not brag about themselves and engage in very little competition among one another. These characteristics may be antithetical to the adoption of performance goals. 
Many communities in rural Appalachia are described by Gjelton (1982) as depressed rural or isolated rural. Gjelten classified rural communities into five different types: high growth, reborn, stable rural, depressed rural, and isolated rural. High growth communities are those located adjacent to growing metropolitan areas, and reborn areas are populated with people who are trying to escape the city. Communities with a strong agricultural base and a stable tax base provided by citizens who participate in the national economy are considered stable rural. Areas with an underdeveloped local economy and characterized by a large migration of its people out of the area are depressed rural, and isolated rural communities are miles from transportation centers and commercial areas.

Because so many communities in rural Appalachia are depressed or isolated rural, it is often difficult to separate the beliefs and values that represent the culture of rural Appalachia from those that represent the culture of poverty. For example, Ford (1983) found that the number of people who wanted their children to stay at home rather than move elsewhere to find work declined as socioeconomic status rose. He also concluded that the attitude of fatalism was related more to poverty than to rural life.

Social class within the Appalachian culture may therefore affect the beliefs and values one adopts. Eller (1982) described how the middle class emerged in Appalachia during the post industrial era. However, he maintained that old cultural patterns continued to exist because of the lack of urbanization during that period. More recently, Plaut (1983) argued that there are two distinct sociocultural systems in Appalachia. There is the "traditional" system tied to kinship and geographic territory, and there is the "modern" system resulting from the increase in industrialization and urbanization of the region. He argued that although the traditional system may not accurately depict the 
social system in Appalachia today, the traditional cultural values often do still exist in preference to the cultural values of the modern system.

Reck (1985) determined that both low socioeconomic status and rurality contributed to the poor performance of many Appalachian schools as measured by norms such as standardized tests, dropout rates, and numbers of college-bound students. After a review of the literature, Nafziger (1971) concluded that the attitudes of Appalachian people toward education are not only related to level of income, but also to family size, occupational aspiration, and family influence. The section that follows will discuss in more detail the attitudes of the Appalachian community toward schools and education.

\section{Attitudes toward schools and education.}

The attitudes of some Appalachian people toward education and the institution of schools contribute to the difficulty of the mission of schools to meet or exceed the norms that are often established based on metropolitan needs. For example, DeYoung (1995a, 1995b) found that some West Virginia parents did not support school efforts to increase graduation rates and encourage college attendance as much as did officials and teachers of local schools. Many parents valued physical work over intellectual pursuits and preferred vocational education that taught professional skills. In addition, on August 27, 2006, an article appeared in the Lexington Herald Leader that focused on the poor state test results for a middle school in rural Appalachian Kentucky (Ismail, 2006). Despite the hard work of the teachers and staff at this school, they seemed to be making very little progress in reaching their targeted goals under the federal No Child Left Behind law (NCLB). Ismail reported that other community members did not worry much about the student achievement problems at the school, and that many rural, low-income 
communities were not concerned with sanctions issued by NCLB. Searching for an answer, Jack Jennings, president of the Center on Education Policy remarked, "Unfortunately, in some poor areas in Kentucky there is a culture that doesn't value education" (p. 10).

In contrast to the remark by Jennings (Ismail, 2006), Shaw, DeYoung, and Rademacher (2004) asserted “...education continues to be highly valued in Appalachia like the rest of the United States" (p. 307). One might explain these rather opposite statements by examining social class. Different beliefs and values may emerge from different social classes in the Appalachian region.

Some Appalachian people do not view schools as institutions in their control, but instead view them as outside intrusions that do not meet their particular needs (Branscome, 1972; Ernst \& Drake, 1972; Schrag, 1972). Schools are often seen as the foremost connection between a rural area and the outside world, with a mission to prepare young people for participation in the national culture rather than the local culture (DeYoung, 1995b). DeYoung reported that the churches in this region are sometimes also critical of school teachings, which negatively influence attitudes toward schools and education. Reck (1980) made the point that the schools in Appalachia are no longer the community run and community oriented schools so often portrayed in the research literature on rural life.

Several studies investigated Appalachian K-12 students' attitudes toward education. Reck (1983) studied the attitudes toward education, educational decisions, and educational self-concept of eighth-grade Appalachian females preparing to enter high school. She concluded that these females did have achievement and educational 
aspirations. They believed that the purpose of schooling was to improve their learning abilities, their employment possibilities and themselves in general. These females reported that their mothers encouraged them to succeed at school, and the rewards given for success were mainly verbal, not monetary. Despite their positive attitudes toward high school, by the middle of their freshman year $35 \%$ of the study participants were struggling. Their problems were attributed to loneliness, feelings of isolation, and lack of personal contact with the high school faculty. In general, it appears that they perceived the high school to be a nonsupportive environment characteristic of a performanceoriented climate.

Social class must be considered as a possible explanation for the results of the Reck (1983) study. According to Payne (1996), school is a middle class institution not well understood by those from the lower class or culture of poverty. The negative attitudes developed by many of the Appalachian students in the Reck study might be the result of class differences rather than cultural differences.

In 1980, Reck conducted a study to compare sixth-grade rural Appalachian students to sixth grade urban non-Appalachian students. She compared them on their general self-concept, behavior, intellectual and school status, physical appearance and attributes, anxiety, popularity, and happiness and satisfaction. The Appalachian sample scored significantly lower on general self-concept, behavior, intelligence and school status, physical appearance and attributes, and happiness and satisfaction. Since no significant relationship was found between self-concept and achievement in either sample, Reck concluded that regardless of achievement, the Appalachian students had lower self-concepts. Additionally, through statistical analysis the school-related items on 
the survey instrument provided the largest contribution to the negative self-concept of the Appalachian children. This suggests that the schools themselves were a large factor in creating the poor self-concepts of these students. However, if schools are middle class institutions where students in poverty struggle with following the hidden rules of this social class (Payne, 1996) then the social class of the students must be considered as a possible factor in the results of this study.

\section{Mathematics in Appalachia}

In 2003 DeYoung embarked on an exploratory study that focused on the “...perceptions, understandings and interpretations of the meaning and use of mathematics among teachers, students and administrators...”(p.1) in rural Appalachian high schools. This researcher expects that many of the preservice elementary teachers who will participate in the present study will be graduates of high schools in the rural Appalachian region of eastern Kentucky, and may therefore hold beliefs and attitudes similar to the students in DeYoung's study. The purpose of his research was to attempt to understand how students and other school personnel in the rural Appalachian region of eastern Kentucky constructed meaning around the subject of mathematics. DeYoung termed this the "social construction" (p. 20) of mathematics. He collected data through ethnographic interviews and observations. The data revealed that many students empathized with the task faced by their mathematics teachers to reach all students. The students did not blame their teachers for any lack of mathematics achievement; instead, they blamed their own lack of interest. It appears that students who did not achieve felt they were lacking in motivation. DeYoung also reported that the mathematics teachers were working hard to improve their teaching skills and student learning, and that 
administrators were appreciative of their efforts, particularly given the high stakes testing requirements that drove district schools.

Research that investigates the effect of various components of the Appalachian culture on teaching and learning mathematics is exceedingly slim despite the fact that mathematics achievement, or lack thereof, of students in Appalachia has been an ongoing problem for many decades. In recent years, the federal government has funded several major initiatives in an attempt to remedy this problem. In 1995, a \$10 million dollar grant funded the Appalachian Rural Systemic Initiative (ARSI) with a mission to improve the mathematics and science education of K-12 students in Appalachia. This initiative was later funded for an additional five years. The Appalachian Collaborative Center for Learning, Assessment, and Instruction in Mathematics (ACCLAIM) was a \$5 million initiative funded in 2001. The two primary goals for this center were: 1) to build mathematics capacity in the region and 2) to improve mathematics teaching in K-12 schools and improve teacher education program in the region. A third program, the Appalachian Mathematics and Science Partnership (AMSP) was initiated in 2003 and funded with $\$ 22$ million for five years. The focus of this organization is to improve the performance of Appalachian students in mathematics and science at the preK-16 levels. Because of some of these programs, more research is underway to investigate aspects of teaching and learning mathematics in the culture of Appalachia. By investigating the motivations and attitudes toward mathematics of Appalachian rural preservice elementary teachers, this study will contribute to that endeavor.

Rural Elementary Teachers 
Many elementary teachers in rural areas are themselves products of the schools and communities they now serve. They are natives of their community and hold rural values; therefore, they are likely to perpetuate culturally-embedded beliefs about mathematics teaching and learning. "The way in which teaching is conducted within a culture is so widely shared that anyone who has grown up in the culture probably could enter a classroom tomorrow and act like a teacher" (Hiebert \& Stigler, 2000, p. 8). Teachers themselves may not be aware of the cultural "script" they use in their teaching. Despite the tendency toward a personal mastery-orientation for females and rural populations, rural teachers are apt to create mathematics classroom environments that are performance-oriented because that was their experience. This type of motivational climate may heighten and perpetuate poor attitudes toward mathematics in their own initially mastery-oriented students.

Where rural education once meant education for citizenship (Theobald, 1997), decades of reform based on urban industrial models such as that of Edward Thorndike (Tomlinson, 1997) have reduced the perception of the goal of mathematics education to that of getting a good job. Thorndike's popular arithmetic book (Thorndike, 1929) stressed rote drill and practice and reduced mathematics to sequences of steps requiring only memorization, and quashing any need for thought. He tied the thousands of exercises in his books directly to vocational and life needs (Tomlinson, 1997). It is probable that mathematics education, especially in rural areas, is still suffering the effects of his work to structure schooling around the methods of industrial management, as well as other education reforms based on metropolitan needs (DeYoung \& Lawrence, 1995). Consequently, rural students may not value mathematics or find it useful in their 
lifeworld and, as a result, develop negative attitudes toward mathematics. Rural elementary mathematics teachers and their cultural script for teaching mathematics are a part of this legacy of reform.

\section{Summary}

The overwhelming majority of elementary preservice teachers are female, and research indicates that females are inclined to adopt mastery goals (Barron \& Harackiewicz, 2001; Fennema, 2000; Peterson \& Fennema, 1985). However, traditional mathematics classes are taught in a performance-oriented classroom climate (Anderman et al., 1999; Turner et al., 1998; Ryan \& Patrick, 2001). The learning environment affects the type of achievement goals that students adopt (Ames \& Archer, 1988; Anderman et al., 1999; Cobb et al., 1991; Kumar, 2004; Nicholls et al., 1990; Patrick et al., 2001; Turner et al.; Urdan et al., 1998; Wood \& Seller, 1997). Anderman et al. reported that the culture of mathematics class enhances and encourages the adoption of performance goals. This creates a mismatch between females' inclination for mastery goals and the promotion of performance goals found in most mathematics classes.

Performance goals can be classified into performance-approach and performanceavoid categories (Dweck \& Elliott, 1983; Elliott \& Harckiewicz, 1996; Urdan et al., 1997). Elliot and Harackiewicz (1996) found that both mastery and performanceapproach goals were favorable to learning, but performance-avoid goals had a negative effect on learning. Research indicates that many preservice teachers are weak in mathematics (Meyer, 1980); as a result, they may develop more performance-avoid goals because of years of instruction in performance-oriented mathematics classrooms. 
Many researchers agree that students pursue multiple goals and that patterns of goals should be examined rather than the adoption of one type of goal over the other (Archer, 1994; Barron \& Harackiewicz, 2001; Harackiewicz, 2000; Meece \& Holt, 1993; Ng, 1999; Turner et al., 1998). Therefore, I hypothesize that most preservice elementary teachers will report levels of achievement goals that are highest in performance-avoid goals, followed by mastery goals, and lowest in performance-approach goals.

The research indicates that many preservice elementary teachers have poor attitudes toward mathematics (e.g. Boswell \& Katz, 1980; McGraw et al., 2006). Considerable research has also examined female attitudes. Fennema and Sherman (1976, 1978) and Iben (1991) reported that females have less confidence than males in the mathematics classroom. Females also tend to see mathematics as less useful than males do, especially as they get older (Fennema \& Sherman, 1976, 1978; Boswell \& Katz, 1980). Iben (1991) and Midgley et al. (1989) found a positive relationship between usefulness and mathematics achievement. Since many preservice teachers are lowachievers in mathematics (Meyer, 1980), this also suggests they perceive mathematics as not useful. Fennema and Sherman found that males believed math to be a male domain more so than females did, but they also reported that for low achieving females this belief was much stronger.

For these reasons I hypothesize that elementary preservice teachers will report poor attitudes toward mathematics. I expect to find low levels of confidence in learning mathematics, a low perception of the usefulness of mathematics, and a high level of perception that mathematics is a subject better suited for males. 
Archer (1994) concluded that a mastery-goal orientation plays a large role in the development of positive attitudes, and research supports her position. For example, students with high levels of mastery goals perceive themselves to be competent (Elliot \& Church, 1997; Meece et al., 1988), report more confidence (Kloosterman, 1988; Seifert, 1995), and demonstrate less anxiety (Urdan et al., 1997). This research supports my hypothesis that there will be a positive relationship between the level of mastery goals and confidence in learning mathematics.

The literature relating mastery goals to the perception of mathematics as a male domain appears to be nonexistent. However, it seems logical to argue that female students who view mathematics as a male domain would not desire to develop competence in that subject. Therefore, I hypothesize that there will be a negative relationship between the level of mastery goals and the perception that mathematics is a male domain.

Ames and Archer (1988) concluded that attitudes were a function of students' perception of a mastery-goal orientation in the classroom. If this is the case, then the mismatch between females' inclination for mastery goals and the promotion of performance goals found in most mathematics classes may promote the development of poor attitudes toward mathematics. However, the type of performance goals adopted may play an important role in attitude development. If performance-approach goals are adopted along with mastery goals, then attitudes may remain positive, but if performanceavoid goals are adopted, then attitudes may become negative. Researchers have found that performance-avoid goals have a negative impact on learning (Elliot \& Church, 1997; Middleton \& Midgley, 1997), and that performance-approach goals do not affect learning 
strategies (Middleton \& Midgley. 1997) especially when mastery goals are strong (Urdan et al., 1997). Therefore, I hypothesize a negative relationship between performanceavoid goals and confidence, but there will be no relationship between performanceapproach goals and any of the constructs of attitude.

While some literature exists regarding the motivation and attitudes of students from different cultures to learn mathematics (Elliott et al, 1999; Elliott, Hufton, Illushin, \& Lauchlin, 2001; Elliott, Hufton, Illushin, \& Willis, 2001; Hufton et al., 2003; Ng \& Renshaw, 2002; Stevenson et al., 1990; Stevenson et al., 1986; Watkins et al., 2002), research that focuses on the rural culture is scant. Howley (2003) and Theobald (1997) argue that rural people are communal rather than individualistic. According to Hyde and Kling (2001) performance goals may be grounded in an individualistic society. This suggests that rural cultures may be more mastery than performance oriented. Other research has also suggested that rural people may be mastery-oriented (Elliott et al., 2001; Freeman \& Anderman, 2005). For this reason I have hypothesized that rural preservice elementary teachers will report higher levels of mastery goals in mathematics than nonrural preservice teachers.

Howley (2002) reports that in Kentucky rural students achieve at a lower level than nonrural students in mathematics. Other research has found a positive relationship between attitude toward mathematics and achievement (Ma \& Kishor, 1997), and some results indicate that this relationship is stronger in females than in males (Aiken, 1976; Fennema \& Sherman, 1976a). This suggests that rural preservice elementary teachers may be lower achievers than nonrural elementary teachers are, and may therefore have poorer attitudes toward mathematics. However, Howley does not disaggregate the data 
according to gender. We do not know whether rural female students achieve at lower levels than nonrural female students. If it is the case that rural students are more masteryoriented that nonrural students and that a positive correlation between mastery goals and attitude exists, then rural preservice elementary teachers may possess more positive attitudes than nonrural elementary teachers. Therefore, I hypothesize that rural preservice elementary teachers will report higher levels of confidence, and be less likely to perceive mathematics as a male domain, than nonrural preservice elementary teachers.

Teachers in rural areas of Kentucky maintain that many of their students and parents of their students do not value education (Hufton et al., 2003). Howley (2003) reports that rural students disconnect the mathematics taught in schools with their rural lifeworld, and rightly so. Motivation to learn school mathematics generally focuses on goals such as preparation for college or personal economic pursuits that often are not relevant to those longing to remain in rural areas. Therefore, I hypothesize that rural elementary preservice teachers will perceive mathematics to be less useful than nonrural elementary preservice teachers.

Research in rural mathematics education is extremely scarce because few mathematics education researchers have an interest in this area (Shultz, 2002). Howley (2003) also maintains that researchers have given little attention to influences in the rural culture that support mathematical learning or create resistance to instruction in mathematics. With this dissertation project I hope to bring some attention to differences that the Appalachian rural culture may make in the attitudes and motivational goals students adopt in learning mathematics. 


\section{CHAPTER III}

\section{METHODOLOGY}

\section{Design}

To investigate differences in the types of motivational goals and attitudes between rural and nonrural preservice elementary teachers this study used a causal-comparative design. The independent variable was locale of preservice elementary teachers' K-12 education. The levels of this variable consisted of four comparison groups: Appalachian rural, other rural, urban, and other nonrural. The groups were compared on six dependent variables. Three variables measured the following achievement goals: mastery, performance-approach, and performance-avoid; and three variables measured the following constructs of attitude toward mathematics: confidence in learning mathematics, usefulness of mathematics, and mathematics as a male domain. These variables are shown in Table 1.

The basic research design is shown below. The letter $\mathrm{N}$ stands for non-random assignment of participants into a group. The letters $X_{1}$ through $X_{4}$ stand for the groups defined by the four levels of the independent variable (locale of preservice elementary teachers' K-12 education). The letter O stands for observation (i.e., measurement) of the six dependent variables. 
$(\mathrm{N})$

(N)

$(\mathrm{N})$

(N)
$\left(\mathrm{X}_{1}\right)$

$\left(\mathrm{X}_{2}\right)$

$\left(\mathrm{X}_{3}\right)$

$\left(\mathrm{X}_{4}\right)$
$\mathrm{O}_{1,2,3,4,5,6}$

$\mathrm{O}_{1,2,3,4,5,6}$

$\mathrm{O}_{1,2,3,4,5,6}$

$\mathrm{O}_{1,2,3,4,5,6}$

Table 1

Variables Used in the Research

Independent variable and levels

Dependent variables

Locale of preservice

elementary teachers' K-12 education:

1. Appalachian rural

2. Other rural

3. Urban

4. Other nonrural
Mastery goal orientation

Performance-approach goal orientation

Performance-avoid goal orientation

Confidence in learning mathematics

Usefulness of mathematics

Mathematics as a male domain

This chapter describes the quantitative methodology used in this dissertation study. First, I describe the sample selection with particular emphasis on definitions of rural, Appalachian rural, urban, and nonrural. Second, I identify and justify the instruments for data collection. Third, I disclose the protocol used to carry out the research plan. Fourth, I explain the statistical analysis procedures.

\section{Sample}

The subjects for this study are best described as a sample of convenience taken from the population of students enrolled in the first mathematics content course required 
for a program leading to certification in elementary education at institutions of higher education in Kentucky. Institutions were selected to insure a number of both rural and nonrural elementary preservice teachers.

A sample size of approximately 160 preservice teachers was necessary with approximately 40 in each of the comparison groups. At a minimum, this sample size allows for detection of a medium effect size when using an alpha level of .05 and a power level of .82 .

In this study, preservice teachers were students who were enrolled in the first mathematics content course required for a program leading to certification in elementary education and who indicated that they intended to pursue a program leading to certification. This definition may differ from that in other literature because these students may not have been officially accepted into a teacher education program and were completing this course as a prerequisite prior to their application.

Defining the groups in a causal-comparative study is a vital part of the design and affects the generalizability of the results (Gay \& Airasian, 2003). This study compared the achievement goals and attitudes toward mathematics of rural preservice elementary teachers to the achievement goals and attitudes of nonrural preservice elementary teachers. However, defining what constitutes "rural" is a problem that continues to plague those interested in rural education and research (U.S. Department of Education, 1994). Since these goals and attitudes develop from past experiences in mathematics classes (Ames \& Archer, 1988; Fishbein \& Ajzen, 1975; Kumar, 2004; Nicholls et al., 1990; Patrick et al., 2001), this study categorized preservice teachers based on the locale of their K-12 schooling experience. Participants in the study reported the names of the 
schools they attended, which allowed the researcher to classify the schools using the categories of the independent variable (e.g., urban, rural).

To determine the rural or nonrural locale of K-12 schools attended by the preservice teachers, the researcher used information from the National Center for Education Statistics (NCES). The NCES classifies elementary, middle and secondary schools by locale codes. These codes were developed by the U.S. Bureau of the Census in the 1980s and they use definitions from the federal Office of Management and Budget (OMB). According to the OMB, a Core Based Statistical Area (CBSA) is "a statistical geographic entity consisting of the county or counties associated with at least one core (urbanized area or urban cluster) of at least 10,000 population, plus adjacent counties having a high degree of social and economic integration with the core as measured through commuting ties with the counties containing the core" (OMB, 2000, p. 82238). The OMB categorizes Core Based Statistical Areas as metropolitan or micropolitan. A Metropolitan Statistical Area (MSA) contains at least one urbanized area that has a population of at least 50,000, and a Micropolitan Statistical Area includes at least one urban area that has a population of at least 10,000 but less than 50,000 .

The eight Locale codes currently used by the NCES are:

1. large city

2. mid-size city

3. fringe of a large city

4. fringe of a mid-size city

5. large town

6. small town 
7. rural, outside CBSA/MSA

8. rural, inside CBSA/MSA.

These codes are referred to as metro-centric locale codes. In May 2006, NCES revised its locale code system. The new system includes 12 codes called urban-centric locale codes. Revisions were made to reflect changes in the U.S. population and allow more precision in describing an area. For example, the new codes include a category for small cities, and they separate rural areas into three groups depending on their distance from an urban area. According to NCES (http://nces.ed.gov) the percent of schools in cities and the percent of schools in rural locales did not change much with the new codes. At the present time, online information to classify schools using the NCES website still uses the older Metro-Centric system. Therefore, this research utilized the eight MetroCentric codes. According to The Rural School and Community Trust, a national nonprofit organization dedicated to the improvement of rural schools and communities (http://www.ruraledu.org), both systems are likely to be used over the next few years.

In this study, the researcher classified as rural all schools designated by NCES as rural, outside CBSA/MSA (Category 7) or rural, inside CBSA/MSA (Category 8). Additionally, schools designated as "small town" by NCES were considered rural for the purposes of this study if the population of the town was less than 10,000. Small towns of this size were included because the high schools and middle schools in these towns are often fed by several rural elementary schools in county school districts, and are the only secondary schools in the district. The limitation on the size of small towns considered rural was invoked because towns with a population of 10,000 or more could be considered as a core for a CBSA/Micropolitan Statistical Area making them akin to other 
nonrural areas. This study designated rural schools located in Appalachian counties as Appalachian rural schools. Rural schools not in Appalachia were referred to as other nonrural schools. A list of counties that comprise Appalachia can be found at the website for the Appalachian Regional Commission (www.arc.gov).

Urban schools were those classified by NCES as large city (Category 1) or midsize city (Category 2). Schools for the other nonrural group included those located in the urban fringe of a large city (Category 3), urban fringe of a mid-size city (Category 4), or in a large town (Category 5). Schools in small towns (Category 6) with populations of 10,000 or greater were also designated as other nonrural. Table 2 provides a summary of the categories of the independent variable.

Categories of preservice teachers were defined based on the number of years of schooling in a given locale group. For this study, an Appalachian rural preservice teacher was a preservice teacher who attended Appalachian rural schools for at least 8 of their 13 years of schooling in grades K-12. If they attended rural schools in counties outside Appalachia for at least eight years, then they were classified as an other rural preservice teacher. This study defined urban and other nonrural preservice teachers in a similar manner: an urban preservice teacher was a preservice teacher who attended urban schools for at least eight years, and other nonrural preservice teachers were those who attended schools in towns and suburbs for at least eight years. This number ensured that the participants attended at least two different schools in their specified locale group.

Differences between rural and nonrural were likely to be more evident between subjects who represent the more extreme ends of these four classifications. Therefore, it 
Table 2

Operational Definitions of Levels of the Independent Variable

Levels of

independent variable Region $\quad$ NCES $^{\mathrm{a}}$ category

1. Appalachian rural $\mathrm{A}^{\mathrm{b}} \quad$ 6. Small town (if population $<10,000$ )

7. Rural, outside CBSA/MSA ${ }^{\mathrm{c}}$

8. Rural, inside CBSA/MSA

2. Other rural

Not-A

6. Small town (if population $<10,000$ )

7. Rural, outside CBSA/MSA

8. Rural, inside CBSA/MSA

3. Urban

A or Not-A

1. Large city

2. Mid-size city

4. Other nonrural

A or Not-A

3. Urban fringe of large city

4. Urban fringe of mid-size city

5. Large town

6. Small town (population $\geq 10,000$ )

${ }^{a}$ NCES $=$ National Center for Education Statistics

${ }^{\mathrm{b}} \mathrm{A}=$ Appalachian, as defined by the Appalachian Regional Commission

${ }^{\mathrm{c}}$ CBSA/MSA $=$ Core Based Statistical Area/Metropolitan Statistical Area 
was prudent for the purpose of this study to select participants with a predominant number of years in one of the four locales. If the sample had yielded an inadequate number of subjects for statistical analysis due to the eight-year attendance requirement, then the number of years would have been reduced to seven. This would still have maintained that a majority of the years of schooling (54\%) would have been spent in a particular locale (e.g., rural). Additionally, it was planned that the number of comparison groups would be reduced by combining two groups together if the sample provided an insufficient number for a particular group. This combining of groups was performed, however, the distinction between rural and nonrural was maintained. Thus, at a minimum, this study provided information on detectable differences in motivational goals and attitudes of preservice elementary teachers who were educated in rural and nonrural locations.

\section{Instrumentation}

The study instruments included a background questionnaire to determine the rural/nonrural educational background of the participants (Appendix B), and a survey designed to measure their achievement goals and attitude toward mathematics (Appendix C). The survey consisted of six scales from established instruments: three designed to measure achievement goal orientation and three developed to measure constructs of attitude toward mathematics. The scales contained statements that the participants ranked on a Likert scale from one to five based on their level of disagreement or agreement with the statement.

The scales that were used to measure achievement goals were selected from a set of subscales called the Patterns of Adapted Learning Scales (PALS; Midgley et al., 
2000). Motivation researchers developed these instruments based on research showing that adaptive or maladaptive patterns of learning are related to an emphasis on either mastery or performance goals (Ames, 1992). Additional research has provided evidence that performance goals can be further divided into approach and avoidance components (Elliot \& Harackiewicz, 1996; Middleton \& Midgley, 1997). The PALS instruments were originally published in 1997, but were revised in 2000 (Midgley et al., 2000). This research used the following scales: Mastery Goal Orientation (Revised), PerformanceApproach Goal Orientation (Revised), and Performance-Avoid Orientation (Revised). The PALS survey also includes scales to measure students' perception of their teacher's goals, students' perception of the classroom goal structure, and a variety of student academic-related perceptions, beliefs, and strategies. These scales were unnecessary for this study. The Cronbach's alphas reported in the PALS Manual (Midgley et al., 2000) are .85 for the Mastery Goal Orientation scale, .89 for Performance-Approach scale, and .74 for the Performance-Avoid scale. Midgley et al. (2000) validated these scales by conducting a confirmatory factor analysis.

The PALS instrument is widely used by many motivation researchers at various grade levels (Anderman et al., 1999; Midgley et al., 1996; Roeser, Midgley, \& Urdan, 1996; Ryan \& Patrick, 2001; Ryan \& Pintrich, 1997; Turner et al., 2002; Urdan et al., 1998), but none of these studies used college students as subjects. Another survey, the Motivated Strategies for Learning Questionnaire (MSLQ), is often used in motivation research with college students. However, reviews from the Buros Institute of Mental Measurement (http://unl.edu/buros) were not favorable for this instrument. The reviewers reported that the reliability data were weak and the validity data were limited. In 
considering use of the PALS instrument, the researcher contacted Eric Anderman from the University of Kentucky, one of the PALS authors, concerning its use with college students. Anderman relayed no concerns about the use of the PALS scales with these subjects if reliability/validity measures were reported (personal communication, 12/16/05). Furthermore, Joseph Petrosko, University of Louisville, commented that the adaptation of the PALS instrument to college students was "not a huge conceptual leap" (personal communication, 12/19/05). The wording of some of the items was changed slightly to make the statements more appropriate for college students. For example, "teacher" was changed to "professor." Other motivation researchers have made similar changes in items to adjust for the age level of the participants (Archer, 1994). Eric Anderman reviewed all wording changes to corroborate the integrity of the scales.

The three scales measuring attitudes were subscales from the Fennema-Sherman Mathematics Attitude Scales (Fennema \& Sherman, 1976b). This instrument was developed as part of a grant from the National Science Foundation and consists of nine subscales that measure well-defined constructs of attitude specifically related to learning mathematics. The subscales are (a) The Attitude toward Success in Mathematics Scale, (b) The Mathematics as a Male Domain Scale, (c) The Mother Scale, (d) The Father Scale, (e) The Teacher Scale, (f) The Confidence in Learning Mathematics Scale, (g) The Mathematics Anxiety Scale, (h) The Effectance Motivation Scale in Mathematics, and (i) The Mathematics Usefulness Scale. The scales can be use as an entire package, individually, or in any combination of two or more pieces.

Intra-author judgment and pilot testing established the content validity of the Fennema-Sherman scales. Data from preliminary administrations of the scales were 
compared to the theoretical framework for each scale and judged for consistency with that framework. Melancon, Thompson, and Becnel (1994) undertook further work to establish the validity of these scales. They investigated the factorial validity using a sample of 174 predominantly female elementary school teachers in an urban public school system. The results of their factor structure analysis were generally favorable. The researchers concluded that scores on these scales are reasonably valid.

The Fennema-Sherman scales have been the most popular tool for measuring attitudes in mathematics since their development (Melancon et al., 1994; Mulhern \& Rae, 1998; Tapia, 2004). They have been used with a variety of age levels from middle school students to adults (Fennema \& Sherman, 1977; Fennema \& Sherman, 1978; Karp, 1988; Kloosterman, 1988).

The Dutton Attitude toward Mathematics Scale (1965) was also considered for use in this study. However, that instrument uses a different format than PALS, making it difficult to mix the survey questions on motivation and attitude into a seamless survey. In addition, the Dutton Scale measures overall feelings toward mathematics and is not divided into subscales measuring constructs of attitude. Furthermore, the wording of this instrument focuses on arithmetic rather than mathematics. Elementary teachers today are required to know and teach more than arithmetic to their students. For these reasons, this instrument was ruled out as a measure of attitude toward mathematics for this study.

The subscales of the Fennema-Sherman instrument used in this research were the Confidence in Learning Mathematics Scale, the Mathematics as a Male Domain Scale, and the Mathematics Usefulness Scale. The Confidence in Learning Mathematics Scale was chosen because self-confidence is a popular variable studied by researchers of 
attitude toward mathematics and motivation in learning mathematics (Fennema \& Sherman, 1976; 1978; Iben, 1991; Kloosterman \& Cougan, 1994; McGraw, Lubienski, \& Strutchens, 2006). It has also been correlated with attributional variables related to motivation (Kloosterman, 1988). The Mathematics as a Male Domain Scale was chosen because most elementary preservice teachers are female. The perception that mathematics is for males may affect not only their own motivation for learning mathematics, but also the motivational climate they create in their own future mathematics classrooms. The Mathematics Usefulness Scale was chosen for its implications for rural preservice teachers. Usefulness of mathematics is a salient issue in rural areas (Howley, 2003).

Forgasz and Leder (1999) claimed that due to changing views over the last two decades, the Mathematics as a Male Domain Scale needed revision. They argued that many people today may actually consider mathematics to be a female domain and the scale does not allow for that. Disagreement with positively worded items on the scale is interpreted to reflect no gender stereotyping. However, the studies on which they based their claim did not use the full 12 -item scale, and Forgasz and Leder conceded that their findings were tentative, but warranted further investigation. For the purpose of this study, the interest is in whether or not preservice elementary teachers (who are predominantly female) view mathematics as a subject for males. Teachers who hold this view may have high levels of personal performance-approach goals and may tend to establish a performance-oriented motivational classroom climate that is more conducive to male learning. Therefore, the Mathematics as a Male Domain Scale is an appropriate instrument for this research. 


\section{Procedures}

Participants were recruited from 4-year public higher education institutions in Eastern and Central Kentucky. Institutions included the University of Louisville, Morehead State University, Eastern Kentucky University, and the University of Kentucky. These universities were chosen to provide an adequate number of both rural and nonrural participants, and also because they were within a reasonable distance from the researcher. The University of Louisville and the University of Kentucky provided a pool of predominantly nonrural preservice teachers, while Morehead State University and Eastern Kentucky University contributed primarily to the rural sample. However, there were both rural and nonrural participants from all universities.

Students who were enrolled in the first mathematics content course required by a program leading to certification in elementary education were invited to participate in the study. This course is generally titled Mathematics for Elementary Teachers, Part I (MET1). The researcher first solicited the cooperation of professors who teach MET1 at the various institutions. This solicitation was accomplished through email and direct phone calls to these professors. Once a professor agreed to cooperate, the researcher scheduled a visit to his or her classroom to enlist the students as participants and administer the instruments.

All students enrolled in the course of the cooperating professors were invited to participate in the research. However, the population of this study was limited to rural and nonrural preservice elementary teachers as defined in this research. Once data was collected and examined, the researcher excluded participants who did not attend school in grades K-12 for at least eight years in one of the four defined locale groups. For 
example, a student who attended an Appalachian rural elementary school for six years, a middle school designated as other nonrural for three years, followed by four years in an other nonrural high school was not an eligible participant. Furthermore, the researcher excluded any students who reported intentions to complete programs of study other than elementary education. The background questionnaire was designed to filter these subjects out of the analysis.

At the visit to the class of the cooperating professors, the researcher told the students they were invited to participate in a research study that was investigating how preservice teachers feel toward mathematics. She explained that their participation involved completing a survey where they would rank from one to five the extent to which they disagreed or agreed with a series of statements. Participants were assured of anonymity and the confidentiality of their responses, and told that their participation was voluntary. (Institutional Review Boards from the University of Louisville, the University of Kentucky, Eastern Kentucky University, and Morehead State University had approved this protocol.) The researcher provided the students a copy of a consent preamble (Appendix A), the background questionnaire, and the motivation/attitude survey. Students who chose to participate completed the forms and each student placed their completed form in a manila envelope placed in a convenient location. Students who did not wish to participate were told to turn in blank forms. This visit took approximately 20-30 minutes and this was the only contact with participants necessary for this research.

Table 3 below displays a timetable for these data collection procedures. 
Table 3

Schedule of Data Collection

Month

Activity

Aug. Sept. Oct. Nov. Dec.

Solicit cooperating professors

X

Classroom visits

X $\quad X$

Organize Data

X $\quad X \quad X$

Analyze Data

X X

Write final chapters of dissertation

X X

\section{Data Analysis}

This study examined the following four research questions.

1. What are the levels of achievement goal orientations - mastery goal orientation, performance-approach goal orientation, and performance-avoid goal orientation - for learning mathematics for preservice elementary teachers?

2. What are the attitudes of preservice elementary teachers toward mathematics as revealed by three measures of attitude: confidence in learning mathematics, usefulness of mathematics, and mathematics as a male domain? 
3. What are the relationships among three measures of achievement goal orientation and three measures of attitude toward mathematics for preservice elementary teachers?

4. Do rural and nonrural preservice elementary teachers differ with respect to goal orientation for learning mathematics and attitudes toward mathematics?

To address these questions, self-report instruments were used to collect data from preservice elementary teachers on three types of achievement goals and three constructs of attitude toward mathematics for a total of six dependent variables. A reliability analysis was conducted to measure the internal consistency of the scales. The achievement goal variables were mastery, performance-approach, and performanceavoid, and the attitude variables were confidence, usefulness of mathematics, and mathematics as a male domain. Data were also collected to distinguish between Appalachian rural, other rural, urban and other nonrural preservice elementary teachers as defined in this research. Locale was the independent variable used in the analysis.

Descriptive statistics were used to describe the motivation and attitude of preservice teachers toward mathematics. Means and standard deviations were calculated on all six dependent variables for the entire sample. A discussion of this analysis addressed Questions 1 and 2. Data from the sample was compared and contrasted with published data on the instruments, in an effort to illuminate the characteristics of the participants in this study.

To assess the relationships among the variables (Question 3), Pearson correlation coefficients were calculated for the bi-variate relationships among the six scale scores that are measured for each participant. Thus, it was possible to report the relationships 
among the measures of achievement goal orientation and measures of attitude toward mathematics.

Next, the sample was divided into the four locale subgroups. These subgroups provided four levels for the independent variable, locale. A 4-group multivariate analysis of variance (MANOVA), $\alpha=.05$, was performed to test the following null hypothesis that the population means for the six dependent variables were equal for the four locale subgroups:

$$
H_{0}:\left(\begin{array}{l}
\mu_{11} \\
\mu_{21} \\
\mu_{31} \\
\mu_{41} \\
\mu_{51} \\
\mu_{61}
\end{array}\right)=\left(\begin{array}{l}
\mu_{12} \\
\mu_{22} \\
\mu_{32} \\
\mu_{42} \\
\mu_{52} \\
\mu_{62}
\end{array}\right)=\left(\begin{array}{l}
\mu_{13} \\
\mu_{23} \\
\mu_{33} \\
\mu_{43} \\
\mu_{53} \\
\mu_{63}
\end{array}\right)=\left(\begin{array}{l}
\mu_{14} \\
\mu_{24} \\
\mu_{34} \\
\mu_{44} \\
\mu_{54} \\
\mu_{64}
\end{array}\right)
$$

Assumptions of homogeneity of variances and covariances were tested as part of the MANOVA procedure. In addition, the researcher examined the shape of the frequency distributions on the dependent variables to assess the normality assumption of MANOVA. If the multivariate result was significant and the null hypothesis was rejected, then appropriate post hoc procedures were performed to reveal which of the variables were factors in the overall significance. The MANOVA and post hoc procedures addressed research Question 4.

\section{Summary}

The purpose of this study was to investigate elementary preservice teachers' achievement goal orientations for learning mathematics and the relationship between those goals and their attitudes toward mathematics. Additionally, this study sought 
differences between the goals and attitudes of rural preservice elementary teachers and the goals and attitudes of nonrural preservice elementary teachers.

Three subscales from the PALS instrument (Midgley, 2000) were used to measure achievement goals, and three subscales from the Fennema-Sherman Mathematics Attitude Scales (Fennema \& Sherman, 1976b) were used to measure constructs of attitude. These self-report instruments were administered to college students enrolled in the first mathematics content course required for a program leading to certification in elementary education. These preservice elementary teachers also completed a background questionnaire to determine their rural/nonrural educational background. Descriptive statistics were used to describe the achievement goals and attitudes toward mathematics held by preservice elementary teachers, and a correlational analysis was performed to examine the relationships among these variables. The sample was divided into four subgroups based on locale: Appalachian rural, other rural, urban, and other nonrural. A four-group MANOVA and appropriate post hoc procedures were performed to reveal differences in the goals and attitudes among these four groups. 


\section{CHAPTER IV}

\section{RESULTS}

This chapter will respond to the four research questions put forth by this dissertation study. First, I describe the sample that resulted from data collection at the four universities. Next, I describe the results of the reliability analysis conducted on each of the six subscales with this sample. Last, I report statistical results and analyze them with respect to the previously stated hypotheses.

\section{Sample}

Data were collected during the first two weeks of classes of the fall semester at four universities in Kentucky. Students completed the survey in their Mathematics for Elementary Teachers classes. The study included 384 participants from four universities as follows: Eastern Kentucky, $n=83$; Morehead State, $n=86$; University of Kentucky, $n$ $=131$; and University of Louisville, $n=84$. Each participant completed the background questionnaire, the three PALS subscales - Mastery goal orientation (revised), Performance-approach goal orientation (revised), and Performance-avoid goal orientation (revised), and the three Fennema-Sherman subscales - Confidence in Learning Mathematics, Mathematics as a Male Domain, and Usefulness of Mathematics.

Using the information provided by the background questionnaire, the researcher used the website of the National Center for Education Statistics (http://www.nces.ed.gov/ccd/schoolsearch/) to find the locale codes used to determine 
the locale level (Appalachian rural, other rural, urban, or other nonrural) for each participant. The website of the U. S. Census Bureau, http://factfinder.census.gov, was used to determine the populations of various communities when the locale code was 6 : small town. Small towns with populations less than 10,000 were deemed rural and those with populations of 10,000 or more were labeled other nonrural. In some cases, the locale level could not be determined from the information provided by participants. For example, some participants listed only the secondary schools they attended and not their elementary background. Other times, the schools that they listed could not be found because they may now be closed or consolidated with other participants did not fall into one of the four discrete locale levels. These participants fell into one of three categories: (a) mixed rural (those who attended a mix of Appalachian rural and other rural schools, but did not have a minimum of eight years in either locale); (b) mixed nonrural (those who attended a mix of urban and other nonrural schools without the required eight years in either locale); and (c) mixed rural/nonrural (those who attended some schools in a rural area and some in a nonrural area, but without at least eight years in either locale.) The numbers of participants for each of the locale levels and other categories were as follows: Appalachian rural, $n=111$; other rural, $n=43$; urban, $n=57$; other nonrural, $n=112$, mixed rural, $n=2$; mixed nonrural, $n=9$; mixed rural $/$ nonrural, $n=18$, and not determinable, $n=32$.

The researcher entered the locale level, other background questionnaire data, and survey responses of all participants into a Statistical Package for the Social Sciences (SPSS v. 14) data file. Descriptive statistics were run on the entire sample. As expected, participants were predominantly female $(88.5 \%)$, and most of the preservice teachers 
were sophomores and juniors (76.6\%). Their ages ranged from 18 to 56 with a mean age of 22 . Over $60 \%$ of the participants indicated they would like to return to their home county to teach.

\section{Reliability Analysis}

A reliability analysis was performed on each of the six subscales. In preparation for this analysis, the negatively worded items of the Fennema-Sherman subscales were recoded in accordance with directions given by the authors of the scale (Fennema \& Sherman, 1976b), and the original scales were reconstructed from the survey instrument. The Cronbach's alpha coefficients for the mastery and performance-avoid goal orientations were higher than those that were reported in the PALS manual (Midgley et al., 2000), and the alpha for the performance-approach subscale was very close to the one reported by these researchers. The Cronbach's alphas for the attitude scales were consistent with those reported by Fennema and Sherman (1976b); however, the alpha for the Mathematics as a male domain subscale was somewhat lower. Fennema and Sherman reported an alpha of .87 for this scale. The reliability coefficients for the present study are shown in Table 4.

After the reliability analysis, participants who indicated they were in a program other than elementary or special education were filtered out of the sample. This reduction left a sample size of 340 . Three hundred seventeen participants were elementary education majors and 23 listed special education as their field of study. The research questions were addressed by an analysis of this reduced sample; however, the actual value of $n$ for any given item may vary due to non-responses on some of the survey questions. 
Table 4

Reliability Coefficients for Scales

\begin{tabular}{lccc} 
Scale & $\begin{array}{c}\text { Number } \\
\text { of items }\end{array}$ & $\begin{array}{c}\text { Cronbach's alpha } \\
\text { coefficient }\end{array}$ & $n$ \\
\hline PALS: Mastery & 5 & .883 & 381 \\
PALS: Performance-approach & 5 & .875 & 378 \\
PALS: Performance-avoid & 4 & .821 & 382 \\
\hline Fennema-Sherman: Confidence & 12 & .956 & 375 \\
Fennema-Sherman: Male domain & 12 & .771 & 375 \\
Fennema-Sherman: Usefulness & 12 & .887 & 377 \\
\hline
\end{tabular}

\section{Research Question 1}

What are the levels of achievement goal orientations - mastery goal orientation, performance-approach goal orientation, and performance-avoid goal orientation - for learning mathematics for preservice elementary teachers?

Subscales from the PALS instrument were used to measure the three achievement goal orientations. The participants ranked these Likert-type subscales from one to five according to their level of disagreement or agreement with the statement. Table 5 includes the means and standard deviations for these subscales. It was hypothesized that these means would be highest in performance-avoid goals followed by mastery goals and then performance-approach goals. $\underline{\underline{\underline{E}}}$ ilts reveal that for these preservice elementary 
teachers the means are highest lastery goals, followed by performance-avoid goals.

However, as hypothesized,

Table 5

Descriptive Statistics for PALS Achievement Goal Orientation Scales

$\begin{array}{llll}\text { Scale } & M & S D & n\end{array}$

Achievement Goal Orientation

Mastery

4.274

.685

338

Performance-approach

2.409

.895

338

Performance-avoid

2.711

.959

338

A repeated measures analysis of variance (ANOVA) performed on these means revealed statistically significant results, Hotelling's Trace $=2.965, F(2,336)=498.176, p$ $<.05$. Pairwise comparisons were then made using paired samples $t$-tests. A Bonferroni-corrected alpha level was used to prevent inflation of Type I error due to three $t$-tests performed. The results of this analysis revealed statistically significant differences between each pair of means $(p<.05)$. Cohen's $d$ was calculated to determine the effect size for each paired samples $t$-test. An effect size is a way to assess the magnitude of a statistically significant result. Cohen's $d$ expresses how many standard deviations difference there is between two sample means (Hinkle, Wiersma, \& Jurs, 2003). According to Hinkle et al. the guidelines for interpreting the effect size using Cohen's $d$ are: small $=.25 \sigma$, medium $=.50 \sigma$, and large $=1.0 \sigma$ or greater. The application of these guidelines indicates a large effect size for the mean difference between mastery and 
performance-approach goals and a large effect size for the mean difference between mastery and performance-avoid goals. The effect size for the performanceapproach/performance-avoid pair was moderate. Table 6 summarizes these results.

Table 6

Paired Samples $t$ - Test Results for Comparisons of PALS Scales

\begin{tabular}{lccccc}
\hline & $\begin{array}{c}\text { Mean } \\
\text { Pair }\end{array}$ & & & & \\
\hline Masference & $S D$ & $t$ & $p$ (2-tailed) & Cohen's $d$ \\
Mastery/Perf-avoid & 1.564 & 1.158 & 24.816 & .000 & 1.350 \\
Perf-app/Perf-avoid & -.302 & .692 & -8.026 & .000 & .437 \\
\hline
\end{tabular}

Research Question 2

What are the attitudes of preservice elementary teachers toward mathematics as revealed by three measures of attitude: confidence in learning mathematics, usefulness of mathematics, and mathematics as a male domain?

Subscales from the Fennema-Sherman Mathematics Attitude Scales were used to measure the three attitude constructs. Each subscale consisted of 12 statements which the participants ranked from one to five based on their level of disagreement or agreement. For analysis, the researcher totaled the responses for each statement in the subscale and calculated the means of these sums.

In addition to reporting the means and standard deviations, the researcher determined if the attitudes toward mathematics of preservice elementary teachers were different from the attitudes of some other group. Thus, a comparative sample of subjects 
who had previously completed the Fennema-Sherman scales was needed. It was hypothesized that, compared to published data on the Fennema-Sherman instrument, preservice teachers in this study also would demonstrate low confidence, a tendency to see mathematics as a male domain, and indicate that they did not perceive mathematics as useful. The sample from a study by Eckard (1995) was selected for use as a comparison group. This study investigated the attitudes toward mathematics of 153 college women enrolled in general studies classes. Since the sample for the present study is predominantly college women, such a female comparison group was desirable. Additionally, the Eckard study provided a sample that included women pursuing a variety of programs of study, making it an appropriate group with which to compare a sample of preservice elementary teachers in order to examine possible differences. Table 7 reveals the means for the attitude subscales from both studies.

One sample $t$-tests were performed using the mean scores from the Eckard (1995) study as comparative values. For example, the null hypothesis for the test on the Confidence scale was $\mu_{\mathrm{PT}}=43.24$, where $\mu_{\mathrm{PT}}$ represented the population mean of preservice teachers in the study and 43.24 was the Eckard study mean. A statistically significant difference was found on Confidence in Learning Mathematics $(p<.001)$. This suggests that this sample of preservice elementary teachers had less confidence in learning mathematics than the female college students of various majors in the study by Eckard. There were no significant differences in the means for the Mathematics as a Male Domain and Usefulness of Mathematics subscales. The results of the $t$-tests are shown in Table 7. 
Table 7

Comparative Statistics on Preservice Teachers and Female College Students from Eckard (1995)

\begin{tabular}{|c|c|c|c|c|c|c|}
\hline \multirow{2}{*}{$\begin{array}{l}\text { Fennema-Sherman } \\
\text { Attitude Scale }\end{array}$} & \multicolumn{2}{|c|}{$\begin{array}{l}\text { Preservice teachers } \\
\qquad(n=340)\end{array}$} & \multicolumn{2}{|c|}{$\begin{array}{l}\text { Eckard study } \\
(n=153)\end{array}$} & \multirow[b]{2}{*}{$t$} & \multirow[b]{2}{*}{$p$ (2-tailed) } \\
\hline & $M$ & $S D$ & $M$ & $S D$ & & \\
\hline Confidence & 40.30 & 12.35 & 43.24 & 12.49 & -4.39 & .00 \\
\hline Male domain & 54.13 & 5.20 & 54.10 & 5.12 & .12 & .91 \\
\hline Usefulness & 49.23 & 7.64 & 48.52 & 8.51 & 1.71 & .09 \\
\hline
\end{tabular}

Research Question 3

What relationships exist, if any, among three measures of achievement goal

orientation and three measures of attitude toward mathematics for preservice elementary teachers?

To examine the relationships among the six variables, Pearson correlation coefficients were computed for the whole sample and separately for rural and nonrural groups. Table 8 displays the correlations for the whole sample.

The table reveals several weak to moderate relationships among the achievement goal orientation and attitude variables. All three constructs of attitude were positively correlated with each other and with a mastery goal orientation. There was also a strong positive correlation between performance-avoid and performance-approach goals ( $r=$ $.724, p<.01)$. 
Table 8

Correlations Among the Subscales for Achievement Goal Orientations and Attitudes

Subscale

1

1. Mastery

$1 \quad .073$

23

45

6

2. Performance - approach

$\begin{array}{lllll} & 1 & .724 * * & .121 * & -.093 \quad .088\end{array}$

3. Performance - avoid

$\begin{array}{llllll} & - & 1 & -.045 & -.068 & .002\end{array}$

4. Confidence

- $\quad-\quad-\quad 1 \quad .269 * * .523 * *$

5. Mathematics as a male domain

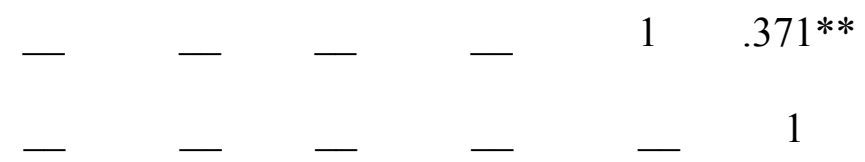

6. Usefulness

${ }^{*} p<.05$, two-tailed. ${ }^{* *} p<.01$, two-tailed.

As hypothesized, there was a significant positive correlation between mastery goal orientation and confidence in learning mathematics $(r=.247, p<.01)$. There was also a significant positive correlation between mastery goals and the mathematics as a male domain variable $(r=.404, p<.01)$. On the Mathematics as a male domain subscale, a high mean indicates a view that mathematics is not a subject just for males. Therefore, this positive correlation means that a mastery goal orientation correlated positively with the view that mathematics is not a subject just for males. This result supports the hypothesis of a negative relationship between mastery goals and the perception that mathematics is a male domain. The correlation analysis also indicated a significant positive correlation between mastery goals and the usefulness of mathematics variable $(r=.581, p<.01)$. 
Prior to the study, the researcher hypothesized that there would be a negative relationship between performance-avoid goals and confidence. Results revealed a negative relationship between these variables $(r=-.045)$, but it was not statistically significant. researcher also hypothesized that no relationship between constructs of attitude and performance-approach goals would exist. However, the results for this analysis showed a slightly positive statistically significant $(r=.121, p<.05)$ relationship between performance-approach goals and confidence in learning mathematics.

Pearson correlation coefficients are presented separately for rural and nonrural groups in Table 9. The rural group included all participants designated as Appalachian rural or other rural, and the nonrural group included those classified as urban or other nonrural. An examination of this table revealed few differences in the relationships among the variables between the two groups. For both groups a mastery goal orientation had a significant positive relationship with each of the mathematics attitude subscales. The strength of these relationships was slightly higher for the rural group on the Usefulness and Male Domain subscales, and slightly higher for the nonrural group on the Confidence subscale. One interesting difference favoring the nonrural group occurred with a low significant correlation $(r=.199, p<.05)$ between a Performance-approach goal orientation and the Confidence subscale. However, for the rural group the direction of the relationship was negative, but not significant $(r=-.008)$. For the rural group there were no statistically significant relationships between Performance-approach or Performance-avoid goals and the three constructs of attitude.

\section{e 9}

Correlations for the Rural group $(n=136)$ and the Nonrural group $(n=150)$

Subscale

1

2116

3

4

5

6 


\section{Research Question 4}

Do rural and nonrural preservice elementary teachers differ with respect to goal orientations for learning mathematics and attitudes toward mathematics?

To address Research Question 4, the sample was divided into the four locale groups: Appalachian rural, other rural, urban and other nonrural. This process further reduced the sample size $(n=286)$ since some of these participants did not fall into one of the four defined locale groups. Table 10 provides means and standard deviations for all the dependent variables in each of the four locale groups. A review of this table does not suggest any outstanding differences among the means of the four locale groups on any of the independent variables.

Table 10

Descriptive Statistics for the Variables for the Four Locale Groups

\begin{tabular}{ccccc}
\hline & & 117 & & \\
& Appalachian & & & \\
rural & Other rural & Urban & Other nonrural \\
Variable & $(n=97)$ & $(n=39)$ & $(n=48)$ & $(n=102)$
\end{tabular}


A 4-group multivariate analysis of variance (MANOVA) was performed to test the null hypothesis that the population means for the six dependent variables (three achievement goal orientations and three constructs of attitude) will be equal for the four locale subgroups comprising the independent variable. Using SPSS the results of four different multivariate tests were reported. These results are given in Table 11. 
Table 11

Multivariate Tests for MANOVA of Locale (Four Levels) Related to Achievement Goal Orientation and Attitude (Six Measures)

\begin{tabular}{lccccc}
\hline Test & Value & $F$ & $p$ & $\begin{array}{c}\text { Partial Eta } \\
\text { Squared }\end{array}$ & $\begin{array}{c}\text { Observed } \\
\text { Power }\end{array}$ \\
\hline Pillai's Trace & .083 & 1.320 & .167 & .028 & .870 \\
Wilks' Lambda & .919 & 1.319 & .168 & .028 & .843 \\
Hotelling's Trace & .086 & 1.317 & .169 & .028 & .869 \\
Roy's Largest Root & .051 & 2.348 & .031 & .048 & .805 \\
\hline
\end{tabular}

The test statistic chosen to interpret the results of a MANOVA often depends on whether the assumptions underlying this procedure have been met. One assumption is that the observations are independent. It is also assumed that the data are normal, and that the variances and covariances in each group are equal (homogeneity of covariance). According to Leech, Barrett, and Morgan (2005), when the data meet these assumptions, the Wilks' Lambda is a solid and commonly used multivariate test. Field (2005) reports that the work of Olson (1976) and Stevens (1979) led Bray and Maxwell (1985) to believe that if assumptions have been violated then Pillai's Trace is the strongest test as long as the sample sizes are equal. Field also maintains that all four tests are comparatively robust to violations of multivariate normality; however, if the assumption of homogeneity of variances and covariances is not met then Roy's Largest Root may be undependable. 
The data for the present study violated several of these assumptions. Histograms constructed to determine the normality of the data revealed that the mastery goal orientation, mathematics as a male domain, and usefulness of mathematics variables were all negatively skewed (see appendices D and E). Therefore, the assumption of normality was violated. Box's Test of Equality of Covariance Matrices was performed to test the assumption that the variances and covariances in each group were equal. The results of this test were significant, $F(63,71505)=1.688, p=.001$, indicating that this assumption was also violated. However, Leech et al. (2005) assert that Box's test may not be accurate if the assumption of normality has been violated.

Additionally, for a MANOVA procedure to be dependable, the number of participants in each level of the independent variable is assumed to be about equal. A common rule is that the $n$ for the largest group should not be more than 1.5 times the $n$ for the smallest group (Leech et al., 2005). According to Leech et al., if the group sizes meet this condition then Box's test should be ignored. In the present analysis, this was not the case. There were only 39 participants in the other rural group (the smallest) compared to 102 participants in the other nonrural group (the largest).

As Table 11 shows, Roy's Largest Root was the only multivariate test that indicated any significant differences among the means $(p<.05)$, and the effect size was small to medium $\left(\eta^{2}=.048\right)$. However, since the homogeneity of variances and covariances assumption was violated this test statistic may be undependable. Univariate $F$ tests based on linearly independent pairwise comparisons revealed that this significant difference resided in the means for confidence in learning mathematics. The Bonferronicorrected pairwise comparisons showed that the mean for confidence in learning 
mathematics was significantly different $(p<.05)$ between the Appalachian rural $(M=$ 3.15) and other nonrural locale $(M=3.54)$ groups, with Appalachian rural preservice teachers having a lower mean. This result suggests that Appalachian preservice elementary teachers may have less confidence in learning mathematics than other nonrural preservice elementary teachers. These analyses found no other significant differences between the four locale groups on any of the other five dependent variables. These results do not support the hypotheses put forward in this study.

As previously stated, histograms revealed that the data for Mastery Goal Orientation, Mathematics as a Male Domain, and Usefulness of Mathematics were negatively skewed. Therefore, $\log 10$ transforms of these three dependent variables were performed in an effort to normalize the data. A second MANOVA using these transformed variables did not change the overall results, Roy's Largest Root $=.059, F(6$, $279)=2.734, p=.013$. Once again, Roy's Largest Root was the only one of the four multivariate tests that indicated any significance difference between the means, and the Bonferroni-corrected pairwise comparisons identified this difference $(p<.05)$ to be between the Appalachian rural group $(M=3.15)$ and the other nonrural group $(M=3.54)$ on the Confidence variable. However, this result should be interpreted cautiously because Box's test indicated once again that the homogeneity of variances and covariances assumption was violated $F(63,71505)=1.454, p=.011$, casting doubt on the fidelity of Roy's Largest Root.

In order to be consistent with the PALS instrument, the previous analyses were performed using the mean scores for both the PALS subscales and the Fennema-Sherman scales. However, the original published data on the Fennema-Sherman scales used the 
means of the sums of the subscales (range $=12-60$ ) rather than the means of the individual survey questions (range $=1-5)($ Fennema $\&$ Sherman, 1976b). For this reason, a third MANOVA was conducted using the sums for the Fennema-Sherman attitude subscales. Since the previous analyses suggested few differences between the four locale groups, this additional analysis combined groups to reduce the levels of the independent variable to two, and made the group sizes similar. The Appalachian rural and other rural levels were combined into one group labeled rural $(n=136)$, and the urban and other nonrural levels were combined into one group labeled nonrural $(n=150)$. Descriptive statistics from this analysis are shown in Table 12.

Box's Test of Equality of Covariance Matrices was significant, $F(21,290727)=$ $2.091, p=.002$ ), indicating that the assumption of equality of variances and covariances between the groups was not met. Since the groups sizes are similar, however, this test can 
be ignored (Leech et al., 2005). No significant differences were found as a result of this MANOVA, Roy's Largest Root $=.041, F(6,279)=1.886, p=.083$. However, Bonferroni-adjusted pairwise comparisons revealed statistically significant differences $(p$ $<.05)$ between the rural and nonrural means for Confidence in Learning Mathematics and between the rural and nonrural means for Mathematics as a Male Domain.

Since the means for the achievement goal variables were virtually the same for both groups and the only differences appeared to exist in the attitude variables, another MANOVA was conducted using only the attitude variables. The multivariate test results of this analysis are shown in Table 13.

Table 13

Multivariate Tests on Three Attitude Variables by Two Groups, Rural and Nonrural

\begin{tabular}{lccccc}
\hline Test & Value & $F$ & $p$ & $\begin{array}{c}\text { Partial Eta } \\
\text { Squared }\end{array}$ & $\begin{array}{c}\text { Observed } \\
\text { Power }\end{array}$ \\
\hline Pillai's Trace & .036 & 3.496 & .016 & .036 & .777 \\
Wilks' Lambda & .964 & 3.496 & .016 & .036 & .777 \\
Hotelling's Trace & .037 & 3.496 & .016 & .036 & .777 \\
Roy's Largest Root & .037 & 3.496 & .016 & .036 & .777 \\
\hline
\end{tabular}

All four multivariate tests indicate significant results, with small to medium effect sizes $\left(\eta^{2}=.036\right)$. Box's test once again revealed a violation of the assumption of homogeneity of variances and covariances, $\mathrm{F}(6,577739)=3.458, p=.002$, but groups sizes were similar. The Bonferroni-adjusted pairwise comparisons indicated significant differences in the confidence and mathematics as a male domain variables. On 
confidence, the means were: nonrural, $M=42.09$; rural, $M=38.62$. On mathematics as a male domain, the means were: nonrural, $M=54.82$; rural, $M=53.49$. These results suggest that rural preservice teachers may have less confidence in learning mathematics than nonrural preservice teachers, and they may be more likely to view mathematics as a subject for males.

\section{Summary}

Research Question 1 focused on the type of achievement goals adopted by preservice elementary teachers. The results of this study indicate that these preservice teachers were significantly higher in mastery goals than performance-approach or performance-avoid goals. The results also indicated that performance-avoid goals were significantly higher than performance-approach goals with this sample.

Research Question 2 focused on preservice elementary teachers' attitudes toward mathematics. The constructs of attitude that were measured were Confidence in Learning Mathematics, Mathematics as a Male Domain, and Usefulness of Mathematics. This sample of preservice teachers was compared to a sample from a previous study that consisted of female college students pursuing a variety of majors. Results indicated that the sample of preservice elementary teachers in this study were significantly less confident in learning mathematics than the sample of female students pursuing a variety of majors (Eckard, 1995). No significant differences between the two samples for mathematics as a male domain or usefulness of mathematics were found.

The third research question focused on the relationship between the three measures of achievement goal orientation and the three measures of attitude. Pearson correlation coefficients were calculated to address this question. Results indicated that 
mastery goals had weak to moderate correlations with all three constructs of attitude that were measured $(p<.01)$. All three constructs of attitude had weak to moderate correlations with each other $(p<.01)$, and performance-approach and performance-avoid goals exhibited a moderate to strong positive correlation $(p<.01)$. There was also a weak positive correlation between performance-approach goals and confidence in learning mathematics $(p<.05)$.

Research Question 4 focused on differences between rural and nonrural preservice teachers on the three measures of goal orientation and the three constructs of attitude. To address this question the researcher divided the participants into four locale groups: Appalachian rural, other rural, urban, and other nonrural. This reduced the sample size ( $n$ $=286$ ), as some participants did not fall into any of these previously defined locale groups. A 4-group MANOVA was performed and of the four multivariate tests computed by SPSS, only Roy's Largest Root indicated significance $\left(p<.05, \eta^{2}=.048\right)$. However, this result may not be dependable since Box's Test indicated that the assumption of homogeneity of variances and covariances was violated. Pairwise comparisons revealed a statistically significant mean difference between the Appalachian rural and other nonrural groups for Confidence in Learning Mathematics with the Appalachian rural preservice teachers having a lower mean.

The sample was then divided into two locale groups: rural and nonrural. A MANOVA using these two levels for the independent variable resulted in no statistical significance on the multivariate tests, but pairwise comparisons indicated statistically significant differences among the means for Confidence in Learning Mathematics and for Mathematics as a Male Domain. The attitude scales were further analyzed by performing 
a MANOVA with two levels for the independent variable (rural and nonrural) and three dependent variables (confidence, male domain, and usefulness). The multivariate tests from this MANOVA were all statistically significant with confidence and male domain once again the source of the significant differences. Pairwise comparisons revealed that the rural group was less confident in learning mathematics and more likely to view mathematics as a subject for males.

The overall results indicated that these preservice elementary teachers were mastery oriented but exhibited low confidence in learning mathematics. The researcher found a positive correlation between a mastery goal orientation and each of the measures of attitude toward mathematics. It appears that Appalachian rural preservice teachers may be less confident in learning mathematics than other nonrural preservice teachers. In addition, rural preservice teachers may have less confidence and may be more likely to view mathematics as a male domain than nonrural preservice elementary teachers. 


\section{CHAPTER V \\ DISCUSSION, LIMITATIONS, AND IMPLICATIONS}

This study investigated elementary preservice teachers' achievement goal orientations for learning mathematics and the relationship between these goals and their attitudes toward mathematics. Teacher actions and attitudes toward mathematics strongly influence the motivations and attitudes of their students (Karp, 1988, 1991; Middleton \& Spanias, 1999). Teachers tend to teach mathematics in the manner they were taught (Ball et al., 2001; Brown \& Smith, 1997; Middleton, 1992), and most were taught using traditional approaches $($ Ball, 1988) that are performance-oriented and tend to favor males (Fennema, 2000). However, research indicates that females are inclined to mastery goals (Fennema, 2000; Peterson \& Fennema, 1985). The result is a possible mismatch between the achievement goals of preservice elementary teachers (largely a female population) and the instructional approach used in the traditional mathematics classroom that may adversely affect their attitude toward mathematics and encourage them to adopt maladaptive performance goals in mathematics classrooms.

This study also explored differences in the types of goals and attitudes adopted by rural preservice elementary teachers and the types of goals and attitudes held by nonrural preservice elementary teachers. Cultural influences seem to affect mathematics learning (Ball et al., 2001; Elliott et al., 1999; Fennema, 2000; Hart \& Allexsaht-Snider, 1996). Instructional approaches may not work equally well with both rural and nonrural students 
due to these cultural differences. Some evidence indicates that rural students have higher levels of mastery goals than their nonrural counterparts (Elliott et al., 2001; Freeman \& Anderman, 2005). This study was exploratory as very little research has been conducted in the area of goal theory with preservice elementary teachers or between rural and nonrural students of mathematics.

The research questions for this study were:

1. What are the levels of achievement goal orientations - mastery goal orientation, performance-approach goal orientation, and performance-avoid goal orientation - for learning mathematics for preservice elementary teachers?

2. What are the attitudes of preservice elementary teachers toward mathematics as revealed by three measures of attitude: confidence in learning mathematics, usefulness of mathematics, and mathematics as a male domain?

3. What relationships exist, if any, among three measures of achievement goal orientation and three measures of attitudes toward mathematics for preservice elementary teachers?

4. Do rural and nonrural preservice elementary teachers differ with respect to goal orientation for learning mathematics and attitudes toward mathematics?

\section{Methodology}

Three subscales from the PALS instrument (Midgley, 2000) were used to measure the three achievement goals--mastery, performance-approach, and performance-avoid. Three subscales from the Fennema-Sherman Mathematics Attitude Scales (Fennema \& Sherman, 1976b) were used to measure the three constructs of attitude toward 
mathematics--confidence in learning mathematics, usefulness of mathematics, and mathematics as a male domain. These self-report instruments were administered to 384 students enrolled in the first mathematics content course required for a program leading to certification in elementary education at the following universities: University of Louisville, University of Kentucky, Morehead State University, and Eastern Kentucky University. The preservice elementary teachers also completed a questionnaire that collected data to determine their rural/nonrural educational background.

The researcher used descriptive statistics to describe the achievement goals and attitudes toward mathematics held by these preservice elementary teachers, and performed correlational analyses to examine relationships among these variables. The preservice teachers were divided into four subgroups based on locale: Appalachian rural, other rural, urban, and other nonrural. A four-group MANOVA and appropriate post hoc procedures were performed to reveal differences in goals and attitudes among these four groups. The attitude scales were analyzed further by performing a MANOVA with two levels for the independent variable - rural (Appalachian rural and other rural) and nonrural (urban and other nonrural), and three dependent variables - confidence, male domain, and usefulness.

The results indicated that the preservice elementary teachers were significantly higher in mastery goals than in performance-approach or performance-avoid goals. The results also indicated that performance-avoid goals were significantly higher than performance-approach goals. The preservice elementary teachers in this study were also significantly less confident in learning mathematics than a comparable sample of female students (Eckard, 1995) pursuing a variety of majors. The correlational analysis revealed 
that mastery goals were weakly to moderately correlated to all three constructs of attitude. A statistically significant difference between the Appalachian rural group and the other nonrural group for confidence in learning mathematics was also found, with the Appalachian rural group displaying less confidence. Results also indicated that rural preservice teachers had less confidence and were more likely to view mathematics as a male domain than nonrural preservice elementary teachers.

\section{Limitations}

Several limitations of this study warrant discussion and suggest the need for caution when interpreting the results. One important limitation involved the methodology used for participant selection. In order to obtain enough rural and nonrural students, this study included data from four universities. The University of Louisville and the University of Kentucky provided the largest percentage of the nonrural participants, and Morehead State University and Eastern Kentucky University contributed the most rural participants. An examination of the data revealed that $21 \%$ of the participants from UK were preservice teachers who attended high school in Fayette County where the university is located. For the $\mathrm{U}$ of $\mathrm{L}$ participants, $50 \%$ of the preservice teachers attended high school in Jefferson County, the home county of that university. As a result, many of the nonrural participants for this study had chosen to remain in their own hometowns. In addition, $64 \%$ indicated a desire to remain in their home county to teach. Therefore, this particular sample of nonrural preservice teachers may represent a subgroup that is more attached to their place than what is typical of the general nonrural population, making them more collectivistic in nature (Hyde \& Kling, 2001), and therefore more mastery-oriented. On the other hand, the rural participants 
may also be atypical of the rural population. At MSU, only $8 \%$ of the preservice teachers attended high school in Rowan County, and at EKU only 6\% were from Madison County. Most of the rural participants were students who already elected to leave their home counties. In rural areas, a decision to attend college is often, by default, a decision to leave home. These students may represent a subgroup of rural students who have overcome cultural programming to stay in one place. Instead, they have chosen to attend college and work toward individual goals. Only 53\% of the rural participants expressed a desire to return to their home county to teach. This subgroup may be representative of rural students who are more individualistic in nature, making them naturally more performance-oriented than what might be typical of rural people in general. As a result, any cultural differences in goal orientations and attitudes toward mathematics may have been confounded.

The large majority of the participants in this study were female, with about $11.5 \%$ male participants. When the researcher removed the participants who were not in a program designed for elementary teachers there were still approximately $7 \%$ of the participants who were male. These male participants were not filtered out of the analysis therefore limiting any results attributed to females or comparisons to female groups.

Another limitation of this study was how the locale groups were defined. These definitions were based on the number of years a student attended school in grades K-12 in a particular locale. For example, the preservice elementary teachers for the Appalachian rural group were placed in this group because they had spent at least eight years of their K-12 education in schools located in a rural area of Appalachia. With this definition, the cultural roots of these students may not have been very deep. It is possible 
that some families had moved to these counties from other locales just prior to students entering school. This definition also neglects the affect that locale during students' first five years of life may have on their motivation and attitude, and it assumes that schools instill the values of the local culture. Further research that seeks to identify cultural differences on educational variables may consider a generational definition of rural. For example, a student would be classified as Appalachian rural if at least two generations of their family had lived in a rural Appalachian county. The results of research using various definitions of rural could differ greatly. Defining rural is an ongoing source of difficulty for rural education researchers (Kannapel \& DeYoung, 1999).

Another limiting factor in this study was that data were collected through selfreport instruments. Although care was taken to ensure the participants that their responses remained anonymous, they may have responded in what they believed acceptable rather than completely open or honest.

A final limitation was that some data were lost because the locale level for the participants could not be determined. Some of the schools listed by the participants on the background questionnaire were not listed on the website of the National Center for Education Statistics (http://www.nces.ed.gov/ccd/schoolsearch/). Possibly these schools had been closed, or consolidated with other schools, since the time that participants attended the schools. These closed or consolidated schools were more likely to have been in rural locales, since many rural schools have closed over the last 50 years (DeYoung, 1995a). Therefore, most of these lost data might have contributed to the rural sample and may have actually contained some of the most culturally acute attitudes.

\section{Discussion}




\section{Goal Orientations}

For the preservice elementary teachers surveyed in this study, an orientation to mastery goals dominated. This result supports the findings of previous research in that females tended to be mastery-oriented (Elliot \& Church, 1997; Fennema, 2000; Peterson \& Fennema, 1985).

Persons who adopt mastery goals seek to increase their competence (Dweck \& Elliot, 1983). Their goal is to acquire knowledge and develop new skills. They value the process of learning and believe that success will come with effort (Ames \& Archer, 1988). Barron and Harrackiewicz (2001) and Barron et al. (2001) reported that masteryoriented college students described greater interest in subject content. Researchers have consistently found positive relationships between mastery goals and intrinsic motivation (Ames \& Archer, 1988; Archer, 1994). That the preservice elementary teachers in this study were high in mastery goals for mathematics is encouraging, because it implies that these future teachers were concerned with mastering the content they will one day teach.

Anderman et al. (1999) found that when mastery-oriented elementary students transitioned to a performance-oriented middle school, they became less mastery-oriented. This result suggests that performance-oriented classroom climates reduce students' level of mastery goals. In the present study, this phenomenon did not appear to be the case. Assuming the prior mathematics instruction of these preservice teachers was primarily traditional, then the years of performance-oriented instruction did not seem to significantly depress their levels of mastery goals. Unfortunately this study did not focus on the type of mathematics instruction received by the preservice teachers during their K- 
12 education. Previous research (Ball et al., 2001), however indicates that it is very likely that the majority experienced much performance-oriented instruction.

According to Middleton \& Spanias (1999), by the time students reach college, their attributions for success have stabilized. These attributions contribute to the type of achievement goals students adopt; therefore, perhaps the achievement goals of college students have also stabilized. In this study, goals were measured only one time. For that reason, it is not possible to discern if the measure of mastery goals reported by preservice teachers was higher or lower than in earlier grades.

Since mastery goals are generally considered best for learning, the result that mastery goals were high and performance goals of both types were relatively low reflect a positive finding. Performance goals have been associated with maladaptive patterns of learning (Ames \& Archer, 1988; Ames, 1992; Archer, 1994; Elliott \& Dweck, 1988); however, Harackiewicz, et al. (2000) argued that if performance goals are limited to the approach variety, then this relationship does not hold. For example, Elliot and Church (1997) found that performance-approach goals were positively associated with better achievement as measured by graded performance in an undergraduate psychology course. On the other hand, the adoption of mastery goals enhanced intrinsic motivation but had no affect on grades. Some researchers have attributed the negative outcomes of performance goals to the avoidance category (Elliot \& Church, 1997; Middleton \& Midgley, 1997). According to Elliot and Church, the adoption of performance-avoid goals had negative effects on both intrinsic motivation and performance. They concluded that the ideal pattern of goals would be the adoption of both mastery and performanceapproach goals combined with a lack of performance-avoid goals. 
Preservice teachers in the present study reported a significantly higher level of performance-avoid goals than performance-approach goals. Additionally, the level of mastery goals was significantly higher than the level of performance-avoid goals. This combination of high mastery goals combined with performance-avoid goals that were significantly higher than performance-approach goals is far from ideal as described by Elliot and Church (1997).

If the pattern of goals found by this study is typical of preservice elementary teachers, then higher levels of performance-avoid goals may explain preservice elementary teachers' poor performance in mathematics content courses despite their high level of mastery goals. Many researchers reported detrimental effects of adopting performance-avoid goals (Elliot \& Church, 1997; Elliot \& Harackiewicz, 1996; Middleton \& Midgley, 1997). These effects included reduced intrinsic motivation, poorer graded performance, reduced task involvement, lower self-efficacy, higher test anxiety, and a tendency to avoid seeking help. Perhaps the mismatch between the personal mastery goals of some students and the performance-oriented instruction typically provided in a traditional mathematics classroom results in the adoption of maladaptive performance-avoid goals by students who are mastery-oriented.

\section{Attitudes}

Previous research indicates that females tend to have less confidence than males in learning mathematics (Fennema \& Sherman, 1976a; 1978; Iben, 1991). The analysis of data in the current study revealed that a predominantly female sample of preservice elementary teachers reported significantly less confidence in learning mathematics than a sample of female college students from a variety of other majors (Eckard, 1995). This 
finding suggests that those who choose elementary education as their field of study are on the low end of confidence levels within a group (females) who are already identified as low in confidence.

That preservice elementary teachers have low confidence in learning mathematics is not encouraging. Teachers with negative attitudes tend to teach mathematics in a performance-oriented style, which leads some of their students to develop low confidence also (Karp 1988; 1991). These teachers utilize limiting instructional strategies, such as focusing on memorized procedures, relying on textbooks, and insisting on finding one right answer. Ma (1999) noted that among the elementary teachers she observed, "Not a single teacher...would promote learning beyond his or her own mathematical knowledge (p. 54)". Therfore, it seems that ineffective mathematics instruction continues a legacy of inadequacy in mathematics content knowledge to another generation of students, continuing the cycle of poor attitudes and insufficient learning.

\section{Attitudes and Goal Orientations}

The preservice teachers in this study revealed a combination of high levels of mastery goals for learning mathematics with low levels of confidence. According to O’Neal, Ernest, McLean, and Templeton (1988), the Fennema-Sherman Confidence in Learning Mathematics Scale and the Mathematics Anxiety Scale appear to measure the same trait. If this is the case, then the low level of confidence reported by preservice teachers is also indicative of mathematics anxiety. According to Harackiewicz and Elliot (1993), mastery-oriented students in performance-oriented classrooms experience anxiety. Since mathematics classes are traditionally performance-oriented, the results of this research support the conclusions of Harackiewicz and Elliott. These results provide 
some evidence that a mismatch between the personal achievement goals of students and the classroom goal orientation result in lower confidence in learning mathematics and therefore, higher levels of mathematics anxiety.

The correlation analysis in this study revealed a high positive correlation between mastery goals and all three constructs of attitude; however, causal direction was not established. This study did not determine if mastery goals lead to positive attitudes or if positive attitudes lead to a mastery goal orientation. Perhaps students high in mastery goals are more confident, believe in the usefulness of mathematics, and are less likely to see mathematics as a male domain, or perhaps confident students who believe mathematics is useful and is a subject suitable for any gender to study adopt mastery goals.

Other researchers found that students with mastery goals demonstrate more confidence (Ames \& Archer, 1988; Elliot \& Church, 1997; Meece et al., 1988; Middleton \& Midgley, 1997; Seifert, 1995; Urdan et al., 1997). The positive correlation between mastery goals and confidence in learning mathematics found in this study supports these previous findings. Students with mastery goals believe that success depends on effort and that occasional failure is part of the learning process. Students without this belief often have their confidence shattered when they encounter a difficult problem that they cannot solve in a routine fashion (Middleton \& Spanias, 1999).

Midgley et al. (1989) found that students' beliefs about the usefulness of mathematics declined when they perceived a less supportive classroom environment. Anderman et al. (1999) revealed that when students moved to a less supportive environment their level of mastery goals dropped. The positive correlation between 
mastery goals and usefulness of mathematics in the present study is consistent with and supports these results.

This study also revealed a positive correlation between mastery goals and the belief that mathematics is a gender-neutral subject. Previous literature relating these variables is virtually nonexistent. The participants in this study were predominantly female, and Fennema and Sherman (1976) found that females were more likely than males to believe that mathematics is gender-neutral. Additionally, Elliot and Church (1997) reported that females in their study were more mastery-oriented than males. Therefore, the results of this research revealing a positive correlation between the two should not be surprising. Logically, females who view mathematics as a male domain would not desire to study it; therefore, this researcher hypothesized a positive relationship between a mastery goal orientation and the belief that mathematics is gender-neutral. The results of this study support this hypothesis.

Positive correlations between a mastery goal orientation and the three constructs of attitude are promising findings for those interested in promoting reform instruction in mathematics classrooms. Mastery goals and positive attitudes are emphasized by the NCTM Standards (1989, 1991, 2000). Quinn (2001) found that a mathematics methods course based on NCTM Standards could improve preservice teacher attitudes toward mathematics. According to Stipek et al. (1998) there was considerable alignment between instruction that promoted mastery goals in students and the type of mathematics instruction called for by NCTM. For example, mastery-oriented instruction focuses on increasing understanding and mastering the content as opposed to just getting the right answer or a good grade. This corresponds to NCTM's call for a greater emphasis on 
conceptual understanding. It appears that by teaching in a manner consistent with the NCTM Standards, teachers can foster a mastery goal orientation in their students and improve their attitudes toward mathematics as well.

\section{Rural/Nonrural Differences}

This study revealed virtually no differences in the means for the achievement goal orientations among Appalachian rural, other rural, urban, and other nonrural groups. When groups were combined and compared dichotomously as rural versus nonrural, no significant differences were found. Achievement goal orientations for learning mathematics of preservice elementary teachers from rural areas compared to preservice elementary teachers from nonrural areas appear to be about the same.

Freeman and Anderman (2005) found that rural middle school students reported higher levels of mastery goals than urban middle school students. This result was not duplicated with this group of predominantly female college students. Harrackiewicz et al. (1997) argued that performance goals may be essential for success in some college classes, particularly when students perceive that competitive performance is valued in the class. Therefore, rural students who attend college may be more likely to adopt performance goals in their college classes despite cultural values that tend toward mastery goals (Jones, 1983). Additionally, mathematics classes are often perceived as performance-oriented classrooms where outperforming others is a measure of success (Anderman et al., 1999; Turner et al., 1998; Ryan \& Patrick, 2001). In the present study, the preservice teachers reported goals specific to their mathematics class, whereas in the Freeman and Anderman study the goals were general to education. Therefore, the rural and nonrural college students in the present study may have been more likely to adopt 
higher levels of performance goals and lower levels of mastery goals than did their sixth grade counterparts in the Freeman and Anderman study, masking any differences between them that may have been evident prior to attending college.

The results of this study also revealed few differences in attitudes toward mathematics among the groups from different locales. The Appalachian rural group, however, reported less confidence in learning mathematics than the other nonrural group. Some evidence that rural preservice elementary teachers have less confidence in learning mathematics and are more likely to view mathematics as a male domain than their nonrural counterparts was revealed in this study. Low confidence is a characteristic of someone with a low self-concept, making these findings consistent with Reck (1980) who found a low self-concept among $6^{\text {th }}$ grade Appalachian rural students compared to urban students. According to Jones (1983) Appalachians have an aversion to competitive environments; therefore, performance-oriented classrooms where traditional mathematics instruction is used may adversely affect the confidence of Appalachian rural students. These findings suggest that those who teach mathematics content courses to students from this region should be sensitive to this characteristic and create a classroom climate where students can feel safe and develop greater confidence in learning and doing mathematics.

\section{Implications}

\section{Teacher Preparation}

This study found that preservice elementary teachers have higher levels of mastery goals than performance goals; however, traditional mathematics classes are performance-oriented (Anderman et al., 1999; Turner et al., 1998; Ryan \& Patrick, 2001). 
The result is a mismatch between the goal orientation of the students and the inherent goal orientation of learning mathematics. This mismatch can create anxiety in masteryoriented students (Harackowicz \& Elliott, 1993). Since motivation researchers generally consider mastery goals best for learning (Ames \& Archer, 1988; Elliot \& Church, 1997; Meece et al., 1988; Middleton \& Midgley, 1997; Seifert, 1995), attempting to change the goal orientation of the students is not recommended. A better solution is to change the motivational climate of the mathematics classroom to one that supports and encourages mastery goals.

To create a mastery-oriented classroom climate in content courses for preservice elementary teachers, mathematics educators can draw on many of the practices that both motivation researchers and mathematics education reformers have suggested for teachers of mathematics. These two independent bodies of literature are remarkably consistent in their recommendations. Many of these suggestions align with the recommendations made by the National Council of Teachers of Mathematics in the Standards $(1989 ; 1991$; 2000), and the Conference Board of the Mathematical Sciences in the report The Mathematical Education of Teachers (CBMS, 2001).

According to Stipek et al. (1998), teachers who establish a learning climate that is positive, affective, and promotes student risk-taking advance the perception of mastery goals in the classroom. The emphasis in these classrooms is on effort, learning and understanding rather than performance. In a safe classroom environment, students feel secure enough to ask and respond to questions, make conjectures, and pose problems. These activities are vital for developing a deep understanding of the mathematics content they will one day teach, as recommended in The Mathematical Education of Teachers 
(CBMS, 2001). Standard Five of Professional Standards for Teaching Mathematics

(NCTM, 1991) recommends this type of learning environment.

Establishing a safe classroom climate requires that instructors respect and value students' ideas, ways of thinking and their mathematical dispositions (CBMS, 2001; NCTM, 1991). Instructors communicate these attitudes to students through the type of discourse they contribute and encourage in the classroom. Too often instructors ask closed questions that have only one correct answer or can be answered with a simple yes or no response. If the student does not immediately deliver the expected answer, the instructor either asks another student or provides the answer. This type of discourse is not supportive and promotes performance goals (Turner et al., 2002). It sends the message to students that having the correct answer is all that matters. If students do not instantly respond with the answer, there are other options for an instructor to consider. One option is to provide some additional wait time, and another option is to ask guiding questions or scaffold the task into smaller parts that build up to the original task or question. This supportive discourse promotes a mastery orientation (Turner et al., 2002), and places the emphasis on learning and improvement rather than just responding with the correct answer (Stipek et al., 1998). The message sent to students is that the teacher values them as learners in a process of continuous learning. Questions designed to promote thinking and understanding of procedures and concepts rather than closed questions (such as those with yes or no answers) will also create a climate where mastery is emphasized (Turner et al., 2002). For example, ask, "Why are common denominators needed when adding fractions?" rather than "Do you need to find a common denominator 
to add fractions?" or "What is $2 / 5+1 / 4$ ?" The central focus should be on sense-making (CBMS, 1991; NCTM, 1991).

Patrick and others (2001) argued that a positive, affective learning environment may be necessary, but it is not sufficient. Teachers must also communicate positive expectations for learning (Kloosterman \& Gorman, 1990; Patrick et al., 2001). According to Turner and her colleagues (2002), the teacher's role in discourse communicates to students whether or not the teacher thinks they are capable of learning. The Equity Principle in the Principles and Standards for School Mathematics (NCTM, 2000), states "Excellence in mathematics education requires equity - high expectations and strong support for all students" (p. 12). The NCTM Standards are based on the assumption that all students can learn to think mathematically (NCTM, 1989; 1991; 2000). A masteryoriented classroom climate is one where students feel capable and confident that success will come with effort. Therefore, instructors must be cautious not to lower their expectations for some students or inadvertently send a signal that they think a student is not capable. Not providing adequate wait time is one way instructors may unintentionally communicate this message to their students (Kloosterman \& Gorman, 1990). Teachers should pose questions that will challenge student thinking (NCTM, 1991) and ask questions to all students, not just those they expect to reply with correct answers (Kloosterman \& Gorman, 1990). Kloosterman and Gorman explained that teachers should praise students only when they deserve it. For example, if a teacher says to a student, "Anna, that's good; you did half the problems right" (p. 379), then the student gets the message that the teacher only expects her to get half right. The 
researchers suggest a better comment would be "Anna, you got half right. That's a good start, but I know you can do better” (p. 379).

Along with these high expectations, instructors should establish that making mistakes is a natural part of the learning process (Ames, 1992; Stipek et al., 1998). Often students believe their mistakes are evidence of their lack of ability to learn mathematics because they never see professors or "good" mathematics students struggle. Turner and her colleagues (2002) recommended that teachers model their thinking processes so that students can learn that being unsure, making errors, and grappling with problems is a normal part of the process of learning. Student errors are learning opportunities. Kloosterman and Gorman (1990) recommended that students be allowed to find their own errors. Supportive discourse can be used to assist the student if necessary. Deciding when it is best to let students struggle, when to guide, or when to directly tell a student a procedure is a skill that all teachers of mathematics should develop (NCTM, 1991).

Another strategy for creating a mastery-oriented classroom climate is to encourage student autonomy (Ames, 1992; Anderman, Maehr, \& Midgley, 1999; Stipek et al., 1998). Students should be encouraged to take control of their own learning. Instructors can accomplish this by providing activities that involve self-evaluation and by giving students some degree of choice in the learning activities in which they participate. Student input on rubrics used for assessment purposes also supports student autonomy. Instructors should refrain from taking students' pencils in order to show them how to work a problem or from giving unnecessary help. These practices undermine student autonomy. 
Mastery-oriented students have learning as their goal; however, teachers can further support this positive inclination by teaching content that is meaningful and personally relevant to the students (Ames, 1992; Anderman et al., 1999). Instructors who strive to establish a classroom with a mastery goal orientation should teach knowledge and skills worth learning in terms of their students' goals and aspirations (Middleton \& Spanias, 2002). The Professional Standards for Teaching Mathematics (NCTM, 1991) also advocates that teachers pose tasks based on student interests and experiences. For preservice elementary teachers in mathematics content courses, this suggests that mathematics educators should connect the content they teach to their students' future aspirations as teachers, particularly as mathematics teachers. Embedding the mathematics content in the context of the education profession will provide a meaningful reason to engage in the learning activities.

Assessment and grading practices often provide vivid insights into the goal orientation of a given classroom. To promote and support mastery goals, instructors should provide substantive, constructive feedback on student papers (Stipek et al., 1998). This type of assessment involves more than just an indication of right or wrong (NCTM, 2000; Stipek, 1998). Additionally, assessment and grading should stress selfimprovement rather than comparing performance with other students (Ames, 1992; Anderman et al., 1999; Kloosterman \& Gorman, 1990). Practices such as posting lists of grades arranged in order from highest to lowest, grading on a curve and encouraging competition are methods that encourage performance goals and are not conducive to a mastery-oriented classroom. Instead, instructors should de-emphasize competition, give 
partial credit, and utilize a grading system where it is possible for all students who learn what they are supposed to learn to earn a good grade (Kloosterman \& Gorman, 1990).

Professors and instructors who teach the mathematics content courses for future elementary teachers should be aware of the results of the present study. This sample of preservice elementary teachers reported a higher level of mastery goals than performance goals. Therefore, teaching mathematics content courses for future elementary teachers in a mastery-oriented classroom climate may be one strategy for improving both attitudes and motivations for learning mathematics in these students. In addition, the use of mastery-oriented instructional strategies may provide a model of teaching that these preservice teachers can use later in their own classrooms. Thus, the cycle of negative attitudes toward mathematics resulting in poor teaching may be broken.

Mathematics educators should also be aware of the possible adoption of performance-avoid achievement goals by preservice elementary teachers revealed by this study. Students with performance-avoid goals seek to avoid unfavorable judgments of their competence (Urdan et al., 1997). As a result, they tend to avoid seeking help when they need it (Middleton \& Midgley, 1997), and they have low competency expectancies, poor intrinsic motivation, lower grades, and a fear of failure (Elliot \& Church, 1997). To discourage these maladaptive performance-avoid goals, mathematics teacher educators should provide a classroom climate that encourages mastery rather than performance goals.

This study also revealed that preservice elementary teachers had less confidence in learning mathematics than a group of female college students pursuing a variety of other majors (Eckard, 1995). This finding is not encouraging for the future mathematics 
education of young children, especially when one considers that becoming confident in one's ability to learn and do mathematics has been one of the educational goals for students established by NCTM since 1989 (NCTM, 1989).

Allowing children to struggle with problems is essential to developing selfconfidence in learning mathematics (Middleton \& Spanias, 1999). Researchers have found that many students pursuing careers in elementary education empathize with young children as they labor to solve a mathematics problem (Ball, 1988; Karp, 1988). Their inclination is to help the child too much, too soon, in an effort to alleviate the child's discomfort. This type of behavior encourages learned helplessness in students (Karp, 1988; Kloosterman, 1984). Young students must learn that occasional failure is acceptable and that occasional errors will naturally occur in the process of learning mathematics. In order for future teachers to understand the importance of this idea, they need to experience the process themselves. Preservice teachers need experiences where they work through their own ideas and errors in order to develop their own confidence in learning and doing mathematics (CBMS, 2001).

Since many preservice elementary teachers struggle with their own confidence in learning mathematics as well as with implementing appropriate practices to develop this confidence in their students, rethinking the structure of mathematics teaching at the elementary school level is advised. One possibility would be to have elementary mathematics specialists teach mathematics in the elementary grades rather than all elementary teachers. Elementary mathematics specialists would elect to become specialists in this area so, logically, only those confident in their abilities would choose to do so. These specialists would receive appropriate content and pedagogical training to 
make them more effective as mathematics teachers. Another possibility is to employ mathematics coaches for elementary teachers in order to provide support and increase their confidence in teaching mathematics. The results of this study join a body of work supporting a move in this direction (Kilpatrick, Swafford, \& Findell, 2001; NCTM, 2000; Reys \& Fennell, 2003).

\section{Future Research}

The results of this study provide direction for future research in the achievement goals and attitudes toward mathematics of preservice elementary teachers.

The group of preservice elementary teachers in this study exhibited a pattern of achievement goals that was highest in mastery, followed by a level of performance-avoid goals that were higher than the level of performance-approach goals. Additional research should be conducted to determine if this pattern of goals is typical of preservice elementary teachers in general. Future studies should assess both the personal goal orientations of preservice teachers in their mathematics content courses and their perceptions of the classroom goal orientation to explore further the effects of a possible misalignment of goals. Another option would be to do an observational study or scrutinize syllabi to determine the predominant goal orientation of the classroom. Assessing students' personal goal orientations at various intervals during mathematics content courses under a variety of instructional methods to evaluate the affect of different teaching strategies on personal achievement goals and perceptions of classroom goals might provide interesting results.

The finding that performance-avoid goals were significantly higher than performance-approach goals is particularly worrisome because research has found that 
these types of goals have detrimental effects on learning (Elliot \& Church, 1997; Elliot \& Harackiewicz, 1996; Middleton \& Midgley, 1997). Additional studies designed to uncover the causes and effects of performance-avoid goal adoption in preservice elementary teachers would potentially improve the preparation of these teachers.

The present study found a weak positive correlation between performanceapproach goals and confidence in learning mathematics, suggesting that performanceapproach goals may be favorable to learning. The results of research in this area have been mixed. Some research revealed positive relationships between performanceapproach goals and variables considered favorable to learning (Elliot \& Harackiewicz, 1996), while other research found that performance-approach goals did not enhance the learning process (Middleton \& Midgley, 1997). One reason for the discrepancy in these results may be age differences among the participants in the studies. Middleton and Midgley studied the performance-approach goals of sixth-grade students, while the participants in the studies by Elliot and Harackiewicz were college students. Future research should investigate the effects of age or grade level on how the adoption of performance-approach goals affects learning.

This study also suggests several directions for future study among differences in achievement goal orientations and attitudes toward mathematics in rural and nonrural preservice teachers. Perhaps applying different definitions for categorizing the participants into the various locale groups would yield different results. For example, a generational definition whereby participants are assigned to a locale only if at least two generations of their family has lived in that locale may offer a more accurate method for identifying participants likely to ascribe to particular cultural values. 
Another recommendation is to replicate this study with K-12 students because cultural differences may be more evident with children. Rural college students may possess some unique characteristics making them an exceptional subgroup of the rural population. For example, they may be less attached to their place because they have already made the decision to leave home to pursue a college education. In this sense, they have overcome the communal/collectivistic orientation of their rural subculture (Hyde \& Kling, 2001). For this reason, they may be less mastery-oriented than the general rural population.

This study sought Appalachian rural participants from Morehead State University in Morehead, KY and Eastern Kentucky University in Richmond, KY. To obtain a sample of college students with deep cultural roots in Appalachia might require enlisting students from smaller, more rural colleges such as Alice Lloyd College in Pippa Passes, KY or Union College in Barbourville, KY, or from community colleges. These students would have chosen to remain as close to home as possible and still pursue a college education.

Lastly, this study focused on differences in attitudes toward mathematics, but it is possible that the lower level of confidence in learning mathematics found for rural students is actually the result of a lower level of confidence for academics in general. Future research should investigate differences in attitudes among rural and nonrural students in other academic areas to determine if this lack of confidence in unique to mathematics.

Summary 
The foundation of this research study was to seek, through an investigation of achievement goal orientations, a possible cause of the deleterious cycle of poor mathematics teaching and negative attitudes toward mathematics unwittingly passed from one generation to the next by elementary teachers. This study found that preservice elementary teachers reported a high level of mastery goals in their mathematics content course. Since mathematics classes are traditionally performance-oriented, this situation presents a mismatch between the predominant goal adopted by these students and traditional mathematics classes. This mismatch could result in negative attitudes toward mathematics and the adoption of maladaptive performance-avoid goals.

Another impetus for this research was to investigate how a cultural context, particularly the rural Appalachian culture, may contribute to the motivation and attitudes of students to learn mathematics. Although this study produced evidence that rural preservice elementary teachers were more likely to view mathematics as a male domain, and may have less confidence in learning mathematics than nonrural preservice elementary teachers, the results were not unequivocal. The more surprising result was the lack of any differences in the achievement goal orientations among participants from the various locale groups. Additional research that utilizes alternative definitions of rural is warranted.

The findings of this study suggest that mathematics educators teaching mathematics content courses to preservice elementary teachers from rural and nonrural locations should create a classroom climate that supports and encourages mastery goals. The characteristics of such a classroom align with the practices suggested by the NTCM Standards $(1989,1991,2000)$. The correlation between mastery goals and positive 
attitudes toward mathematics found by this study suggests that teaching mathematics in this manner may improve the attitudes of these future teachers and therefore increase the likelihood that they will become lifelong learners of mathematics. Preservice elementary teachers instructed in this manner will take positive attitudes and a model of mathematics teaching that centers on student learning with them to their future classrooms. In doing so they will break the cycle of passing on negative attitudes and strategies for mathematics teaching that may promote maladaptive performance-avoid goals. With this cycle broken, we can meet the challenge of rising expectations for K-12 mathematics education. 


\section{REFERENCES}

Aiken, L. R., Jr. (1972a). Research on attitudes toward mathematics. The Arithmetic Teacher, 19, 229-234.

Aiken, L. R., Jr. (1972b). Biodata correlates of attitudes toward mathematics in three age and two sex groups. School Science and Mathematics, 72, 386-395.

Aiken, L. R., Jr. (1976). Update on attitudes and other affective variables in learning mathematics. Review of Educational Research, 46(2), 293-311.

Ames, C. (1984). Achievement attributions and self-instructions under competitive and individualistic goal structures. Journal of Educational Psychology, 76(3), 478487.

Ames, C. (1992). Classrooms: Goals, structures, and student motivation. Jouranl of Educational Psychology, 84(3), 261-271.

Ames, C., \& Archer, J. (1988). Achievement goals in the classroom: Students' learning strategies and motivation processes. Journal of Educational Psychology, 80, 260267.

Anderman, E. M., Maehr, M. L., \& Midgley, C. (1999). Declining motivation after the transition to middle school: Schools can make a difference. Journal of Research and Development in Education, 32, 131-147.

Anderman, L. H., Patrick, H., \& Ryan, A. M. (2004). Creating adaptive motivational environments in the middle grades. Middle School Journal, May, 33-39. 
Archer, J. (1994). Achievement goals as a measure of motivation in university students. Contemporary Educational Psychology, 19, 430-446.

Ball, D. L. (1988). Unlearning to teach mathematics. For the Learning of Mathematics, $8(1), 40-48$.

Ball, D. L., Lubienski, S. T., \& Mewborn, D. S. (2001). Research on teaching mathematics: The unsolved problem of teachers' mathematical knowledge. In V. Richardson (Ed.), Handbook of research on teaching ( $4^{\text {th }}$ ed.). New York: Macmillan.

Banks, J. H. (1964). Learning and teaching arithmetic. Boston, MA: Allyn \& Bacon. Barron, K. E., \& Harackiewicz, J. M. (2001). Achievement goals and optimal motivation: Testing multiple goal models. Journal of Personality and Social Psychology, 80(5), 706-722.

Barron, K. E., Harackiewicz, J. M., \& Tauer, J. M. (2001, April). The interplay of ability and motivational variables over time: A 5 year longitudinal study of predicting college student success. Paper presented at the Annual Meeting of the American Educational Research Association, Seattle, WA.

Batteau, A. (1983). Appalachia and the concept of culture: A theory of shared misunderstandings. In B. Ergood \& B. E. Kuhre (Eds.), Appalachia: Social context past and present, Ohio University, Athens, OH: Kendall/Hunt Publishing. Boekaerts, M. (2002). Motivation to learn. Educational practices series. (Report No. IAE/IBE-Ser-10). Geneva, Switzerland: International Bureau of Education. (ERIC Document Reporduction Service No. ED470681) 
Boswell, S. L., \& Katz P. A. (1980). Nice girls don't study mathematics. (Grant No. NIE-G-78-0023). Washington, DC: National Institute of Education. (ERIC Document Reproduction Service No. ED188888)

Bowd, A. D., \& Brady, P. H. (2003). Gender differences in mathematics anxiety among preservice teachers and perceptions of their elementary and secondary school experience with mathematics. Alberta Journal of Educational Research, 49(1), 24-36.

Branscome, J. (1972). The crisis of Appalachian youth. In D. S. Walls \& J. B. Stephenson (Eds.), Appalachia in the sixties. Lexington, KY: University Press of Kentucky.

Bray, J. H. \& Maxwell, S. E. (1985). Multivariate analysis of variance. Sage university paper series on quantitative applications in the social sciences, 9-07-054. Newbury Park, CA: Sage.

Brown, C. A., \& Smith, M. S. (1997). Supporting the development of mathematical pedagogy. The Mathematics Teacher, 90(2), 138-143.

Bulmahn, B. J., \& Young, D. M. (1982). On the transmission of mathematics anxiety. Arithmetic Teacher, 30(3), 55-56.

Bush, W. (2002). Culture and mathematics: An overview of the literature with a view to rural contexts. (Working Paper No. 2). Athens, OH: Ohio University, Appalachian Collaborative Center for Learning, Assessment and Instruction in Mathematics Research Initiative. Retrieved from http://www.acclaim$\underline{\text { math.org/resworking.aspx }}$ 
Bush, W. (2003). Bridging the Gap between Culture and Mathematics: The Appalachian Perspective. (Occasional Paper No. 2). Athens, OH: Ohio University, Appalachian Collaborative Center for Learning, Assessment and Instruction in Mathematics Research Initiative. Retrieved from http://www.acclaim-math.org/docs/occasional_papers/OP_02_Bush.pdf

Carr, M. (1996). Metacognitive, motivational, and social influences on mathematics strategy use. In M. Carr (Ed.) Motivation in mathematics (pp. 89-111). Cresskill, NJ: Hampton Press.

Cobb, P., Wood, T., Yackel, E., Nicholls, J., Wheatley, G., Trigatti, B., et al. (1991). Assessment of a problem-centered second-grade mathematics project. Journal for Research in Mathematics Education, 22(1), 3-29.

Coladarci, T. (2003). Reflections at 35,000 feet: An open letter to the ACCLAIM doctoral cohort. (Occasional Paper No. 5). Athens, OH: Ohio University, Appalachian Collaborative Center for Learning, Assessment and Instruction in Mathematics Research Initiative. Retrieved on March 23, 2006 from http://www.acclaim-math.org/docs/occasional_papers/OP_05_Coladarci.pdf

Conference Board of the Mathematical Sciences. (2001). The mathematical education of teachers. Providence, RI: American Mathematical Society.

DeYoung, A. J. (2003). The Social Construction of Rural Mathematics. (Working Paper No. 17). Athens, $\mathrm{OH}$ : Ohio University, Appalachian Collaborative Center for Learning, Assessment and Instruction in Mathematics Research Initiative. Retrieved from http://www.acclaim-math.org/resworking.aspx 
DeYoung, A. J. (1995a). The life and death of a rural American high school: Farewell Little Kanawha. New York: Garland.

DeYoung, A. J. (1995b). Constructing and staffing the cultural bridge: The school as change agent in rural Appalachia. Anthropology \& Education Quarterly, 26(2), 168-192.

DeYoung, A. J., \& Lawrence, B. K. (1995). On Hoosiers, Yankees, and Mountaineers. Phi Delta Kappan, 77(2), 105-114.

Di Cintio, M. J. (1996). Student motivation and the complexity of mathematics instruction before and after the middle school transition. Dissertation Abstracts International, 57(04), 1482. (UMI No. 9628071)

Diener, C. I., \& Dweck, C. S. (1978). An analysis of learned helplessness: Continuous changes in performance, strategy, and achievement cognitions following failure. Journal of Personality and Social Psychology, 36, 451-462.

Dweck, C. S. (1975). The role of expectations and attributions in the alleviation of learned helplessness. Journal of Personality and Social Psychology, 31(4), 674685.

Dweck, C. S., \& Elliott, E. S. (1983). Achievement motivation. In E. M. Hetherington (Ed.), Socialization, personality, and social development (pp. 643-691). New York: Wiley.

Dweck, C. S., \& Leggett, E. L. (1988). A social-cognitive approach to motivation and personality. Psychological Review, 95(2), 256-273. 
Eckard, J. S. (1995). Correlating attitudes and college majors among undergraduate women. (Master's thesis, Chadron State College, 1995). (ERIC Document Reproduction Service No. ED382123).

Eller, R. D. (1982). Miners, millhands, and mountaineers: Industrialization of the Appalachian South, 1880-1930. Knoxville, TN: The University of Tennessee Press.

Elliot, A. J., \& Church, M. A. (1997). A hierarchical model of approach and avoidance achievement motivation. Journal of Personality and Social Psychology, 72(1), $218-232$.

Elliot, A. J., \& Harackiewicz, J. M. (1996). Approach and avoidance achievement goals and intrinsic motivation: A mediational analysis. Journal of Personality and Social Psychology, 70(3), 461-475.

Elliott, J. G., Hufton, N., Hildreth, A., \& Illushin, L. (1999). Factors influencing educational motivation: a study of attitudes, expectations and behaviour of children in Sunderland, Kentucky and St. Petersburg. British Educational Research Journal, 25(1), 1999.

Elliott, J. G., Hufton, N., Illushin, L., \& Lauchlan, F. (2001). Motivation in the junior years: International perspectives on children's attitudes, expectations and behaviour and their relationship to educational achievement. Oxford Review of Education, 27(1), 37-68.

Elliott, J. G., Hufton, N., Illushin, L., \& Willis, W. (2001). “The kids are doing all right": Differences in parental satisfaction, expectation and attribution in St. Petersburg, Sunderland, and Kentucky. Cambridge Journal of Education, 31(2), 179-204. 
Ergood, B., \& Kuhre, B. E. (Eds.). (1983). Appalachia: Social context past and present. Dubuque, Iowa: Kendall/Hunt.

Ernest, J. (1976). Mathematics and sex. American Mathematical Monthly, 83(8), 595614.

Ernst, H. \& Drake, C. (1972). The lost Appalachians. In D. S. Walls \& J. B. Stephenson (Eds.), Appalachia in the sixties. Lexington, KY: University Press of Kentucky.

Fennema, E. (2000). Gender Equity for Mathematics and Science. A presentation at Gender Equity for Mathematics and Science: A conference of the Woodrow Wilson leadership program for teachers, Princeton, NJ. (ERIC Document Reproduction Service No. ED446968)

Fennema, E., \& Sherman, J. (1976a, February). Sex-related differences in mathematics learning: Myths, realities and related factors. Paper presented at the annual meeting of the American Association for the Advancement of Science, Boston, MA. (ERIC Document Reproduction Service No. ED129633)

Fennema, E. \& Sherman, J. (1976b). Instruments designed to measure attitudes toward the learning of mathematics by females and males. JSAS Catalog of Selected Documents in Psychology, 6, 31 .

Fennema, E. \& Sherman, J. (1977). Sex-related differences in mathematics achievement, spatial visualization, and affective factors. American Educational Research Journal, 14(1), 51-71.

Fennema, E. \& Sherman, J. (1978). Sex-related differences in mathematics achievement and related factors: A further study. Journal for Research in Mathematics Education, 9(3), 189-203. 
Field, A. (2005). Discovering statistics using SPSS ( $2^{\text {nd }}$ ed.). Thousand Oaks, CA: SAGE Publications.

Fishbein, M. (1963). An investigation of the relationships between beliefs about an object and the attitude toward that object. Human Relations, 16, 233-240.

Fishbein, M., \& Ajzen, I. (1975). Belief, attitude, intention, and behavior. An introduction to theory and research. Reading, MA: Addison-Wesley.

Ford, T. R. (1983). The passing of provincialism. In B. Ergood \& B. E. Kuhre (Eds.), Appalachia: Social context past and present, Ohio University, Athens, OH: Kendall/Hunt Publishing.

Forgasz, H. J., \& Leder, G. C. (1999). The Fennema-Sherman Mathematics as a Male Domain Scale reexamined. Journal for Research in Mathematics Education, 30(3), 342-348.

Foss, D. H., \& Kleinsasser, R. C. (1996). Preservice elementary teachers' views of pedagogical and mathematical content knowledge. Teaching \& Teacher Education, 12(4), 429-442.

Freeman, R. M., \& Anderman, L. H. (2005). Changes in mastery goals in urban and rural middle school students. Journal of Research in Rural Education, 20(1). Retrieved 8/23/2005 from http://www.umaine.edu/jrre/20-1.pdf

Gay, L. R. \& Airasian, P. (2003). Educational research: Competencies for analysis and applications ( $7^{\text {th }}$ ed.). Columbus, OH: Merrill Prentice Hall.

Gibson, H. L., Brewer, L. K., Magnier, J., McDonald, J. A., \& Van Strat, G. A. (1999, April). The impact of an innovative user-friendly mathematics program on preservice teachers' attitudes toward mathematics. Paper presented at the Annual 
Meeting of the American Educational Research Association, Montreal, Quebec, Canada.

Gjelten, T. (1982). A typology of rural school settings. Washington, DC: U. S. Department of Education. (ERIC Document Reproduction No. ED215858)

Good, T. L., \& Brophy, J. E. (1991). Looking in classrooms. New York, NY: Harper Collins.

Gottfried, A. E. (1985). Academic Intrinsic Motivation in Elementary and Junior High School Students. Journal of Educational Psychology, 77, 631-645.

Gottfried, A. E. (1990). Academic intrinsic motivation in young elementary school children. Journal of Educational Psychology, 82(3), 525-538.

Grouws, D. A., \& Lembke, L. O. (1996). Influential factors in student motivation to learn mathematics: The teacher and classroom culture. In M. Carr (Ed.), Motivation in mathematics (pp. 39-62). Cresskill, NJ: Hampton Press, Inc.

Harackiewicz, J. M., \& Elliot, A. J. (1993). Achievement goals and intrinsic motivation. Journal of Personality and Social Psychology, 65(5), 904-915.

Harackiewicz, J. M., Barron, K. E., Tauer, J. M., Carter, S. M., \& Elliot, A. J. (2000). Short-term and long-term consequences of achievement goals: Predicting interest and performance over time. Journal of Educational Psychology, 92(2), 316-330.

Hart, L. E., \& Allexsaht-Snider, M. (1996). Sociocultural and motivational contexts of mathematics learning for diverse students. In M. Carr (Ed.) Motivation in mathematics (pp. 89-111). Cresskill, NJ: Hampton Press. 
Hiebert, J., Gallimore, R., \& Stigler, J. (2002). A knowledge base for the teaching profession: What would it look like and how can we get one? Educational Researcher, 31(5), 3-15.

Hiebert, J., \& Stigler, J. W. (2000). A proposal for improving classroom teaching: Lessons from the TIMSS video study. The Elementary School Journal, 101(1), 3-20.

Hinkle, D. E., Wiersma, W., Jurs, S. G. (2003). Applied Statistics for the behavioral sciences $\left(5^{\text {th }}\right.$ ed.). Boston: Houghton-Mifflin.

Howley, C. (2002). Research about mathematics achievement in the rural circumstance. (Working Paper No. 4). Athens, OH: Ohio University, Appalachian Collaborative Center for Learning, Assessment and Instruction in Mathematics Research Initiative. Retrieved from http://www.acclaimmath.org/docs/working_papers/WP_04_Howley.pdf

Howley, C. (2003, April). The lifeworld makes mathematics education rural: Implications for math education research. Paper presented at the Annual Meeting of the National Council of Teachers of Mathematics, San Antonio, TX.

Hufton, N. R., Elliott, J. G., \& Illushin, L. (2003). Teachers' beliefs about student motivation: Similarities and differences across cultures. Comparative Education, 39(3), 367-389.

Hyde, J. S., \& Kling, K. C. (2001). Women, motivation, and achievement. Psychology of Women Quarterly, 25, 364-378.

Iben, M. F. (1991). Attitudes and mathematics. Comparative Education, 27(2), 135-152. Retrieved December 11, 2005, from Academic Search Premier database. 
Ismail, R. H. (2006, August 27). For rural schools, hurdles are huge. The Lexington Herald-Leader, pp. A1, A10.

Jackson, D. N. (1974). Manual for the Personality Research Form. Port Huron, MI: Research Psychologists Press.

Jones, A. R., Jr., \& Taylor, L. (1963). White youth from low-income rural families achievement milieu and agribusiness opportunities. Washington, DC: National Committee for Children and Youth. (ERIC Document Reproduction Service No. ED012663)

Jones, L. (1969). Appalachia - discovered and rediscovered. TheBerea Alumnus. Special Issue: A salute to Appalachia. Berea, KY: Berea College.

Jones, L. (1983). Appalachian values. In B. Ergood \& B. E. Kuhre (Eds.), Appalachia: Social context past and present, Ohio University, Athens, OH: Kendall/Hunt Publishing.

Kamii, C. (1994). Young children continue to reinvent arithmetic- $3^{\text {rd }}$ grade: implications of Piaget's theory. New York: Teachers College Press.

Kannapel, P. J., \& DeYoung, A. J. (1999). The rural school problem in 1999: A review and critique of the literature. Journal of Research in Rural Education,15(2), 6779.

Kaplan, A., \& Midgley, C. (1997). The effect of achievement goals: Does level of perceived academic competence make a difference? Comtemporary Educational Psychology, 22, 415-435.

Karabenick, S. A. (2004). Perceived achievement goal structure and college student help seeking. Journal of Educational Psychology, 96(3), 569-581. 
Karp, K. S. (1988). The teaching of elementary school mathematics: The relationship between how mathematics is taught and teacher attitudes. Dissertation Abstracts International, 49(08), 2138. (UMI No. 8816727)

Karp, K. S. (1991). Elementary school teachers' attitudes toward mathematics: The impact on students' autonomous learning skills. School Science and Mathematics, 91(6), 265-270.

Kilpatrick, J., Swafford, J., \& Findell, B. (Eds.). (2001). Adding it up: Helping children learn mathematics. Washington, DC: National Academy Press.

Kloosterman, P. (1984, April). Attribution theory and mathematics education. Paper presented at the annual meeting of the American Educational Research Association, New Orleans.

Kloosterman, P. (1988). Self-confidence and motivation in mathematics. Journal of Educational Psychology, 80(3), 345-351.

Kloosterman, P., \& Cougan, M. C. (1994). Students' beliefs about learning school mathematics. The Elementary School Journal, 94(4), 375-388.

Kloosterman, P., \& Gorman, J. (1990). Building motivation in the elementary mathematics classroom. School Science and Mathematics, 90(5), 375-382.

Kloosterman, P., Raymond, A. M., \& Emenaker, C. (1996). Students' beliefs about mathematics: A three-year study. The Elementary School Journal, 97(1), 39-56.

Kumar, R. (2004). Multicultural education and achievement goal theory: School reforms for education in a culturally pluralistic society. In P. R. Pintrich \& M. L. Maehr (Eds.), Motivating students, improving schools: The legacy of Carol Midgley. San Diego, CA: Elsevier Ltd. 
Leech, L. L., Barrett K. C., \& Morgan, G. A. (2005). SPSS for intermediate statistics: Use and interpretation ( ${ }^{\text {nd }}$ ed.). Mahwah, NJ: Lawrence Erlbaum.

Lepper, M. R. (1988). Motivational considerations in the study of instruction. Cognition and Instruction, 5,289-309.

Lepper, M. R., \& Henderlong, J. (2000). Turning "play" into "work" and "work" into "play": 25 years of research on intrinsic versus extrinsic motivation. In C. Sansone \& J. M. Harachiewicz (Eds.), Intrinsic and extrinsic motivation: the search for optimal motivation and performance. San Diego: Academic Press.

Ma, L. (1999). Knowing and teaching elementary mathematics. Mahwah, NJ: Lawrence Erlbaum Associates.

Ma, X., \& Kishor, N. (1997). Assessing the relationship between attitude toward mathematics and achievement in mathematics: A meta-analysis. Journal for Research in Mathematics Education, 28(1), 26-47.

Maehr, M. L., \& Nicholls, J. G. (1980). Culture and achievement motivation: A second look. In N. Warren (Ed.), Studies in cross-cultural psychology (Vol. 2, pp. 221267). New York: Academic Press.

Magnusson, J., \& Perry, R. P. (1992). Academic help-seeking in the university setting: The effects of motivational set, attributional style, and help source characteristics. Research in Higher Education, 33(2), 227-245.

McGraw, R., Lubienski, S. T., \& Strutchens, M. E. (2006). A closer look at gender in NAEP mathematics achievement and affect data: Intersections with achievement, race/ethnicity, and socioeconomic status. Journal for Research in Mathematics Education, 37(2), 129-150. 
McLeod, D. B. (1992). Research on affect in mathematics education: A reconceptualization. In D. A. Grouws (Ed.), Handbook of Research on Mathematics Teaching and Learning. New York: Macmillan.

McMillan, J. H. (1976). Factors affecting the development of pupil attitudes toward school subjects. Psychology in the Schools, 13(3), 322-325.

Meece, J. L., Blumenfeld, P. C., \& Hoyle, R. H. (1988). Students' goal orientations and cognitive engagement I classroom activities. Journal of Educational Psychology, $80(4), 514-523$.

Meece, J. L., \& Holt, K. (1993). A pattern analysis of students' achievement goals. Journal of Educational Psychology, 85(4), 582-590.

Meece, J. L., Wigfield, A., \& Eccles, J. S. (1990). Predictors of math anxiety and its influence on young adolescents' course enrollment intentions and performance in mathematics. Journal of Educational Psychology, 82(1), 60-70.

Melancon, J. G., Thompson, B., \& Becnel, S. (1994). Measurement integrity of scores from the Fennema-Sherman Mathematics Attitudes Scales: The attitudes of public school teachers. Educational and Psychological Measurement, 54(1), 187192.

Meyer, R. A. (1980). Attitudes of elementary teachers toward mathematics. (ERIC Document Reproduction Service No. ED190388).

Middleton, J. A. (1992). Teachers'vs. students’ beliefs regarding instrinsic motivation in the mathematics classroom: A personal constructs approach. Paper presented at the annual meeting of the American Educational Research Association, San Francisco, CA. 
Middleton, J. A., \& Spanias, P. A., (1999). Motivation for achievement in mathematics: Findings, generalizations, and criticisms of the research. Journal for Research in Mathematics Education, 30, 65-88.

Middleton, M. J., \& Midgley, C. (1997). Avoiding the demonstration of lack of ability: An underexplored aspect of goal theory. Journal of Educational Psychology, 89(4), 71-718.

Midgley, C., Arunkumar, R., \& Urdan, T. C. (1996). "If I don't do well tomorrow, there's a reason": Predictors of adolescents' use of academic self-handicapping strategies. Journal of Educational Psychology, 88(3), 423-434.

Midgley, C., Feldlaufer, H., \& Eccles, J. S. (1989). Student/teacher relations and attitudes toward mathematics before and after the transition to junior high school. Child Development, 60, 981-992.

Midgley, C., Maehr, M. L., Hruda, L. Z., Anderman, E., Anderman, L., Freeman, K. E., et al. (2000). Manual for the Patterns of Adaptive Learning Scales. Retrieved on March 15, 2006 from http://www.umich.edu/ pals/manuals.html

Mulhern, F., \& Rae, G. (1998). Development of a shortened form of the FennemaSherman Mathematics Attitudes Scales. Educational and Psychological Measurement, 58(2), 295-306.

National Council of Teachers of Mathematics. (1989). Curriculum and evaluation standards for school mathematics. Reston, VA: Author

National Council of Teachers of Mathematics. (1991). Professional standards for teaching mathematics. Reston, VA: Author 
National Council of Teachers of Mathematics. (2000). Principals and standards for school mathematics. Reston, VA: Author

Nafziger, A. J. (1971). Analysis of attitudes relative to education in the Appalachian region. Discussion paper prepared for Public Schools for Cooperative Research (PSCR Superintendent's Conference, University of Tennessee, Knoxville. (ERIC Document Reproduction Service No. ED048982)

Ng, C. (1999, November). Adolescent students' multiple goals in learning mathematics. Paper presented at the Annual Meeting of the Australian Association for Research in Education and the New Zealand Association for Research in Education, Melbourne, Australia.

Ng, C. \& Renshaw, P. (2002). Self-schema, motivation and learning: A cross-cultural comparison. In D. M. McInerney \& S. Van Etten (Eds.), Research on Sociocultural Influences on Motivation and Learning: Volume 2, Greenwich, CT: Information Age Publishing.

Nicholls, J. G. (1983). Conceptions of ability and achievement motivation: A theory and its implications for education. In S. G. Paris, G. M. Olson, \& H. W. Stevenson (Eds.), Learning and motivation in the classroom (pp. 211-237). Hillsdale, NJ: Erlbaum.

Nicholls, J. G., Cobb, P., Wood, T., Yackel E., \& Patashnick, M. (1990). Assessing students' theories of success in mathematics: Individual and classroom differences. Journal for Research in Mathematics Education, 21(2), 109-122.

Office of Management and Budget. (2000). Standards for defining metropolitan and micropolitan statistical areas. Federal Register, 65, No. 249, 82228-82238. 
Olson, C. L. (1976). On choosing a tst statistic in multivariate analysis of variance. Psychological Bulletin, 83, 579-586.

O’Neal, M. R., Ernest, P. S., McLean, J. E., \& Templeton, S. M. (1988). Factorial validity of the Fennema-Sherman mathematics attitudes scales. Paper presented at the annual meeting of the Mid-South Educational Research Association, Louisville, KY.

Pajares, F., \& Miller, M. D. (1994). Role of self-efficacy and self-concept beliefs in mathematical problem solving: A path analysis. Journal of Educational Psychology, 86(2), 193-203.

Patrick, H., Anderman, L. H., Ryan, A. M., Edelin, K. C., \& Midgley, C. (2001). Teachers' communication of goal orientations in four fifth-grade classrooms. The Elementary School Journal, 102(1), 35-58.

Payne, R. (1996). A framework for understanding poverty. Highlands, TX: aha! Process, Inc.

Peterson, P. L., \& Fennema, E. (1985). Effective Teaching, Student Engagement in Classroom Activities, and Sex-Related Differences in Learning Mathematics. American Educational Research Journal, 22, 309-335.

Phillips, R. B., Jr. (1973). Teacher attitude as related to student attitude and achievement in elementary school mathematics. School Science and Mathematics, 73(6), 501507.

Plaut, T. (1983). Conflict, confrontation, and social change in the regional setting. In. A. Batteau (Ed.), Appalachia and America: Autonomy and regional dependence (pp. 267-284). Lexington, KY: The University Press of Kentucky. 
Quinn, R. J. (2001). Effects of mathematics methods courses on the mathematical attitudes and content knowledge of preservice teachers. The Journal of Educational Research, 91(2), 108-113.

Randhawa, F. S. , \& Michayluk, J. O. (1975). Learning environment in rural and urban classrooms. American Educational Research Journal, 12(3), 265-279.

Reck, U. M. L. (1980). Self-concept, school, and social setting: A comparison of rural Appalachian and urban non-Appalachian sixth graders. Journal of Educational Research, 74(1), 49-54.

Reck, U. M. L. (1983). Educational attitudes, decisions, and self-concept: A look at Appalachian females moving from the elementary experience to the high school experience. Paper presented at the Annual Meeting of the American Educational Research Association, Montreal, Quebec, Canada. (ERIC Document Reproduction Service No. ED232793)

Reck, U. M. L. (1985). The effects of ethnicity, class and residence on student participation in a southern Appalachian high school. Paper presented at the Annual Meeting of the American Educational Research Association, Chicago, IL. (ERIC Document Reproduction Service No. ED256548)

Reys, B. J. \& Fennell, F. (2003). Who should lead mathematics instruction at the elementary school level? A case for mathematics specialists. Teaching Children Mathematics, 9(5), 277-282.

Reyes, L. H., \& Stanic, G. M. A. (1988). Race, sex, socioeconomic status and mathematics. Journal for Research in Mathematics Education, 19(1), 26-43. 
Robinson, M. L. (1975). Attitudes and achievement: A complex relationship. (ERIC Document Reproduction Service No. ED111678)

Roeser, R. W., Midgley, C., \& Urdan, T. C. (1996). Perceptions of the school psychological environment and early adolescents' psychological and behavioral functioning in school: The mediating role of goals and belonging. Journal of Educational Psychology, 88(3), 408-422.

Ryan, A. M., \& Patrick, H. (2001). The classroom social environment and changes in adolescents' motivation and engagement during middle school. American Educational Research Journal, 38, 437-460.

Ryan, A. M. \& Pintrich, P. R. (1997). "Should I ask for help?" The role of motivation and attitudes in adolescents' help seeking in math class. Journal of Educational Psychology, 89(2), 329-341.

Sansone, C., \& Harackiewicz, J. M. (2000). Controversies and new directions - is it déjà vu all over again? In C. Sansone \& J. M. Harachiewicz (Eds.), Intrinsic and extrinsic motivation: the search for optimal motivation and performance. San Diego: Academic Press.

Sax, G. (1989). Principles of Educational and Psychological Measurement and Evaluation ( ${ }^{\text {rd }}$ ed.). Belmont, CA: Wadsworth.

Schrag, P. (1972). The school and politics. In D. S. Walls \& J. B. Stephenson (Eds.), Appalachia in the sixties. Lexington, KY: University Press of Kentucky.

Seifert, T. L. (1995). Academic goals and emotions: A test of two models. The Journal of Psychology, 129(5), 543-552. 
Shaw, T. C., DeYoung, A. J., \& Rademacher, E. W. (2004). Educational attainment in Appalachia: Growing with the nation, but challenges remain. Journal of Appalachian Studies, 10(3), 307-329.

Shultz, J. E. (2002). Mathematics Education in Rural Communities in Light of Current Trends in Mathematics Education. (Working Paper No. 1). Athens, OH: Ohio University, Appalachian Collaborative Center for Learning, Assessment and Instruction in Mathematics Research Initiative. Retrieved from http://www.acclaim-math.org/resworking.aspx

Stevens, J. P. (1979). Comment on Olson: Choosing a test statistic in multivariate analysis of variance. Psychological Bulletin, 86, 355-360.

Stevenson, H. W., Lee, S., Chen, C., Stigler, J. W., Hsu, C., \& Kitamura, S. (1990). Contexts of achievement: A study of American, Chinese, and Japanese children. Monographs of the Society for Research in Child Development, 55(1-2, Serial No. 221).

Stevenson, H. W., Lee, S., \& Stigler, J. W. (1986). Mathematics achievement of Chinese, Japanese, and American children. Science, 231, 693-699.

Stigler, J. W., \& Hiebert, J. (1999). The Teaching Gap. New York: Simon \& Schuster. Stipek, D., Salmon, J. M., Givvin, K. B., Kazemi, E., Saxe, G., \& MacGyvers, V. L. (1998). The value (and convergence) of practices suggested by motivation research and promoted by mathematics education reformers. Journal for Research in Mathematics Education, 29(4), 465-488. 
Tapia, M. (2004). An instrument to measure mathematics attitudes. Academic Exchange Quarterly, 8(2). Retrieved on December 14, 2005 from http://www.rapidintellect.com/AEQweb/cho253441.htm

Taylor, P. M. (2002). Implementing the standards: Keys to establishing positive professional inertia in preservice mathematics teachers. School Science and Mathematics, 102(3), 137-142.

Theobald, P. (1997). Teaching the Commons. Boulder, CO: Westview Press.

Thorndike, E. L. (1929). The psychology of arithmetic. New York: The Macmillan Company.

Tishlor, A. G. (1980, November). A study of attitude - Treatment interaction in mathematics with preservice elementary school teachers. Paper presented at the Annual Meeting of the Mid-South Educational Research Association, New Orleans, LA.

Tomlinson, S. (1997). Edward Lee Thorndike and John Dewey on the science of education. Oxford Review of Education, 23(3), 365-384.

Turner, J. C., Meyer, D. K., Cox, K. E., Logan, C., DiCintio, M., \& Thomas, C. T. (1998). Creating Contexts for Involvement in Mathematics. Journal of Educational Psychology, 90(4), 730-745.

Turner, J. C., Midgley, C., Meyer, D.K., Gheen, M., Anderman, E. M., Kang, Y., et al. (2002). The classroom environment and students' reports of avoidance strategies in mathematics: a multimethod study. Journal of Educational Psychology, 94, 88-106. 
Turner, J. C., Thorpe, P. K., \& Meyer, D. K. (1998). Students' reports of motivation and negative affect: A theoretical and empirical analysis. Journal of Educational Psychology, 90, 758-771.

Urdan, T., Midgley, C. \& Anderman, E. M. (1998). The role of classroom goal structure in students' use of self-handicapping strategies. American Educational Research Journal, 35(1), 101-122.

Urdan, T., Pajares, F., \& Lapin, A. Z. (1997, March) Achievement goals, motivation, and performance: A closer look. Paper presented at the Annual Meeting of the American Educational Research Association, Chicago, IL.

U. S. Department of Education. (1994). The Condition of Education in Rural Schools. Retrieved March 12, 2006 from http://ww.ed.gov/PressReleases/071994/rural.html.

Vachon, J. (1984). Attitudes: Their relationship with intelligence and achievement and their evaluation. Paper presented at the NATO Scientific Affairs Committee Advance Study Institute, Athens, Greece. (ERIC Document Reproduction Service No. ED274667)

Walberg, H. J. (1969). Social environment as a mediator of classroom learning. Journal of Educational Psychology, 60(6), 443-448.

Watkins, D., McInerney, D., Lee, C., Akande, A., \& Regmi, M. (2002). Motivation and learning strategies: A cross-cultural perspective. In D. M. McInereny \& S. Van Etten (Eds.), Research on Sociocultural Influences on Motivation and Learning: Volume 2, Greenwich, CT: Information Age Publishing. 
Weller, J. E. (1965). Yesterday's people: Life in contemporary Appalachia. Lexington, KY: University of Kentucky Press.

Wilkins, J. L. M. (2002). The impact of teachers' content knowledge and attitudes on instructional beliefs and practices. In Proceedings of the Annual Meeting of the North American Chapter of the International Group for the Psychology of Mathematics Education, 1-4, Athens, GA.

Wood, T., \& Sellers, P. (1997). Deepening the analysis: longitudinal assessment of a problem-centered mathematics program. Journal for Research in Mathematics Education, 28, 163-186. 
Appendix A

Motivation and attitude of preservice teachers toward mathematics: Is rural relevant?

March 1, 2006

Dear Student:

You are being invited to participate in a research study by answering the attached survey about your motivation and attitude toward mathematics. There are no known risks for your participation in this research study. The information collected may not benefit you directly. The information learned in this study may be helpful to others. The information you provide will be used to examine the relationship between motivation and attitude towards mathematics, and investigate the effects of a rural culture on these constructs. Your completed survey will be stored at the home of the researcher. The survey will take approximately 15-20 minutes to complete.

Individuals from the Department of Teaching and Learning, the Institutional Review Board (IRB), the Human Subjects Protection Program Office (HSPPO), and other regulatory agencies may inspect these records. In all other respects, however, the data will be held in confidence to the extent permitted by law. Should the data be published, your identity will not be disclosed.

Taking part in this study is voluntary. By completing this survey you agree to take part in this research study. You do not have to answer any questions that make you uncomfortable. You may choose not to take part at all. If you decide to be in this study you may stop taking part at any time. If you decide not to be in this study or if you stop taking part at any time, you will not lose any benefits for which you may qualify.

If you have any questions, concerns, or complaints about the research study, please contact Christie Perry at 606-782-7734.

If you have any questions about your rights as a research subject, you may call the Human Subjects Protection Program Office at (502) 852-5188. You can discuss any questions about your rights as a research subject, in private, with a member of the Institutional Review Board (IRB). You may also call this number if you have other questions about the research, and you cannot reach the research staff, or want to talk to someone else. The IRB is an independent committee made up of people from the University community, staff of the institutions, as well as people from the community not connected with these institutions. The IRB has reviewed this research study.

If you have concerns or complaints about the research or research staff and you do not wish to give your name, you may call 1-877-852-1167. This is a 24 hour hot line answered by people who do not work at the University of Louisville.

Sincerely,

Signature of the Investigator

Signature of the Co-Investigator 
Appendix B

\section{Background Questionnaire}

1. Student Status (circle one, or fill in on the blank line for Other if appropriate):

Freshman Sophomore Junior Senior Graduate Other

2. Program of Study (check one):

Elementary education

Middle school education

Other (please explain)

3. Gender (circle one): male female

4. Age:

5. School Background

Please complete the following table to provide information about your K-12 education. Please include all schools you attended.

Please Print

\begin{tabular}{|l|l|l|l|}
\hline Name of school attended & County & State & $\begin{array}{c}\text { Grades attended } \\
(\mathrm{K}, 1,2, \text { etc. })\end{array}$ \\
\hline & & & \\
\hline & & & \\
\hline & & & \\
\hline & & & \\
\hline & & & \\
\hline & & & \\
\hline & & & \\
\hline & & & \\
\hline & & & \\
\hline
\end{tabular}




\section{Appendix C}

On the following pages is a series of statements. There are no correct answers for these statements. They have been set up in a way that permits you to indicate the extent to which you agree or disagree with the ideas expressed. Suppose the statement is:

\section{I like mathematics. $1122 \quad 3 \quad 4 \quad 5$}

As you read the statement, you will know whether you agree or disagree. If you strongly disagree, circle 1 on your sheet. If you disagree but with reservations, that is, you do not fully agree, circle 2 . If you agree with the idea, indicate the extent to which you agree by circling 4 for agree or circling 5 if you strongly agree. However, if you neither agree nor disagree, that is, you are not certain, circle 3 for undecided. Also, if you cannot answer a question, circle 3.

Do not spend much time with any statement, but be sure to answer every statement. Some of the items will seem similar to others, but they are different, so do answer all items. There are no "right" or "wrong" answers. The only correct responses are those that are true for you. Whenever possible, let the things that have happened to you help you make a choice.

1. Mathematics is a worthwhile and necessary subject.

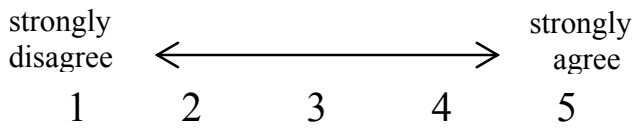

2. It's important to me that I learn a lot of new concepts in math this semester.

3. Males are not naturally better than females in mathematics.

4. Math has been my worst subject.

5. I'll need a firm mastery of mathematics for my future work.

6. It's important to me that other students in my math class think I am good at my math work.

$\begin{array}{lllll}1 & 2 & 3 & 4 & 5 \\ 1 & 2 & 3 & 4 & 5\end{array}$

8. Women certainly are logical enough to do well in mathematics.

12

9. Most subjects I can handle $\mathrm{OK}$, but I have a knack for flubbing up math.

10. It's important to me that I don't look stupid in math class.

11. I expect to have little use for mathematics when I get out of school.

12. Mathematics is for men; arithmetic is for women.

13. I think I could handle more difficult mathematics.

14. One of my goals in math is to learn as much as I can.

$\begin{array}{lllll}1 & 2 & 3 & 4 & 5\end{array}$


15. In terms of my adult life, it is not important for me to do well in mathematics in college.

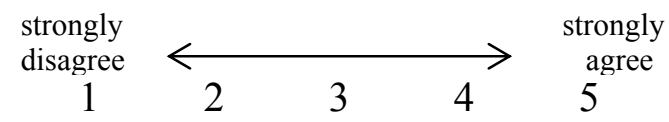

16. I would expect a woman mathematician to be a masculine type of person.

17. I am sure that I can learn mathematics.

18. One of my goals in my math class is to show others that I'm good at my math work.

19. Girls who enjoy studying math are a bit peculiar.

20. I am sure I could do advanced work in mathematics.

21. One of my goals is to keep others from thinking I'm not smart in math class.

22. Taking mathematics is a waste of time.

23. Girls can do just as well as boys in mathematics.

24. I have a lot of self-confidence when it comes to math.

25. One of my goals is to master a lot of new math skills this semester.

26. I'll need mathematics for my future work.

27. I would trust a woman just as much as I would trust a man to figure out important calculations.

$\begin{array}{lllll}1 & 2 & 3 & 4 & 5\end{array}$

28. For some reason even though I study, math seems unusually hard for me.

$\begin{array}{lllll}1 & 2 & 3 & 4 & 5\end{array}$

29. One of my goals in math class is to show others that math work is easy for me.

30. I will use mathematics in many ways as an adult.

$\begin{array}{lllll}1 & 2 & 3 & 4 & 5\end{array}$

31. When a woman has to solve a math problem, it is feminine to ask a man for help.

32. I'm no good in math.

$\begin{array}{lllll}1 & 2 & 3 & 4 & 5\end{array}$

33. It's important to me that I thoroughly understand my math class work.

34. I study mathematics because I know how useful it is.

$\begin{array}{lllll}1 & 2 & 3 & 4 & 5\end{array}$

$\begin{array}{lllll}1 & 2 & 3 & 4 & 5\end{array}$

$\begin{array}{lllll}1 & 2 & 3 & 4 & 5\end{array}$

$\begin{array}{lllll}1 & 2 & 3 & 4 & 5\end{array}$


35. I would have more faith in the answer for a math problem solved by a man than a woman.

36. Generally, I have felt secure about attempting mathematics.

37. One of my goals is to look smart in comparison to the other students in my math class.

38. Mathematics is of no relevance to my life.

39. Females are as good as males in geometry.

40. I can get good grades in mathematics.

$\begin{array}{lllll}1 & 2 & 3 & 4 & 5\end{array}$

41. It's important to me that my math professor doesn't think that I know less than other in class.

$\begin{array}{lllll}1 & 2 & 3 & 4 & 5\end{array}$

42. I see mathematics as a subject I will rarely use in my daily life as an adult.

$\begin{array}{lllll}1 & 2 & 3 & 4 & 5\end{array}$

43. Studying mathematics is just as appropriate for women as for men

44. I do not think I could complete advanced mathematics.

45. It's important to me that I improve my math skills this semester.

46. Mathematics will not be important to me in my life's work.

47. It's hard to believe a female could be a genius in mathematics.

48. It's important to me that I look smart compared to others in my math class.

$\begin{array}{lllll}1 & 2 & 3 & 4 & 5\end{array}$

49. One of my goals in math class is to avoid looking like I have trouble doing the work.

50. I am not the type to do well in math.

$\begin{array}{lllll}1 & 2 & 3 & 4 & 5 \\ 1 & 2 & 3 & 4 & 5\end{array}$




\section{Appendix D}
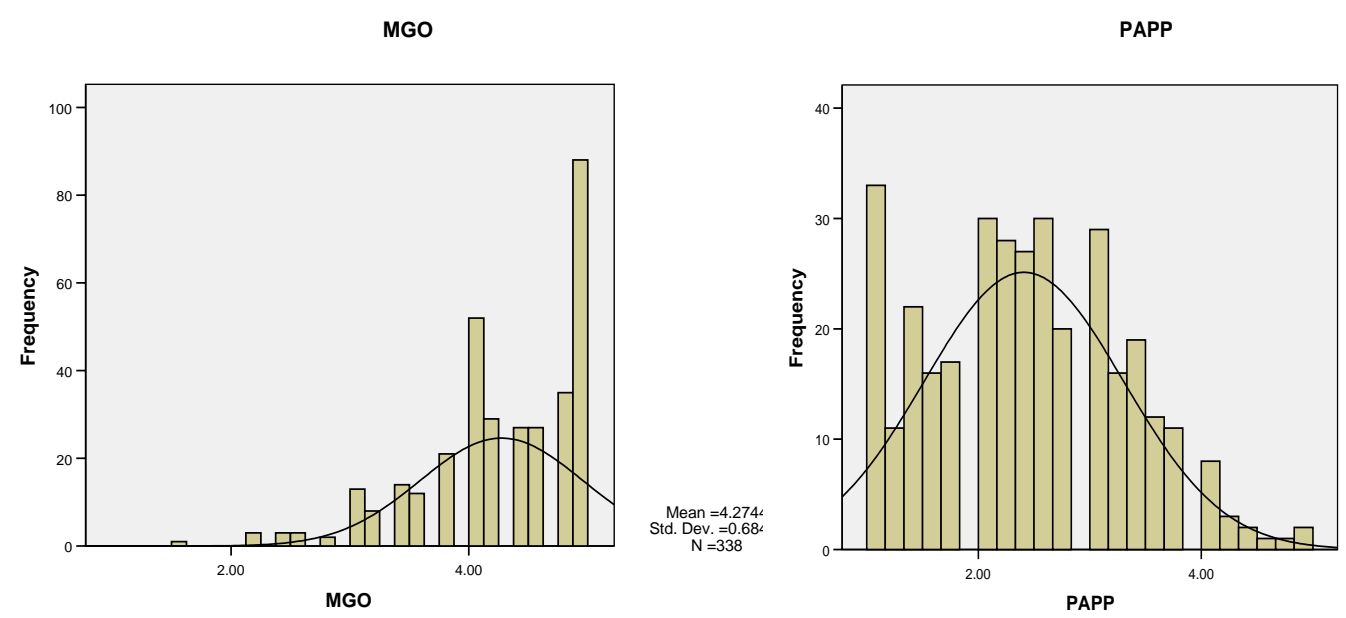

Mean $=2.4089$
Std. Dev. $=0.09467$
$N=338$

PAVO

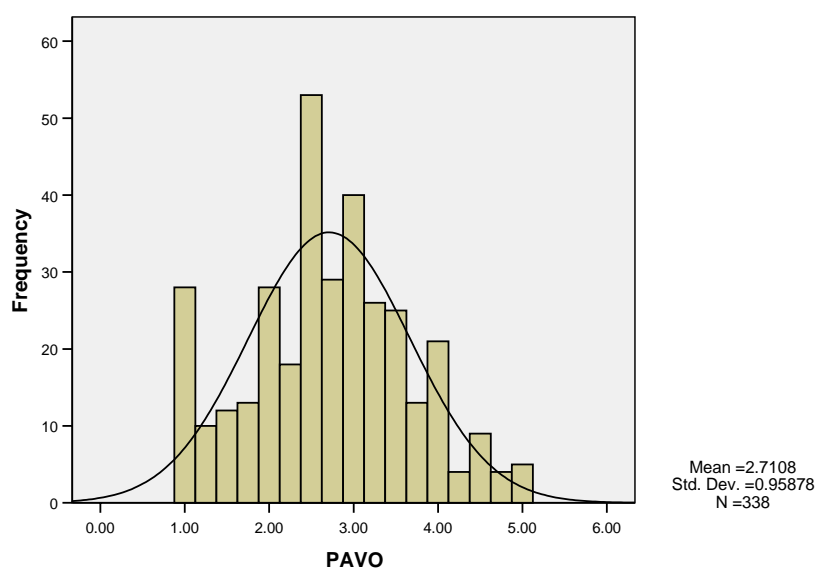




\section{Appendix E}
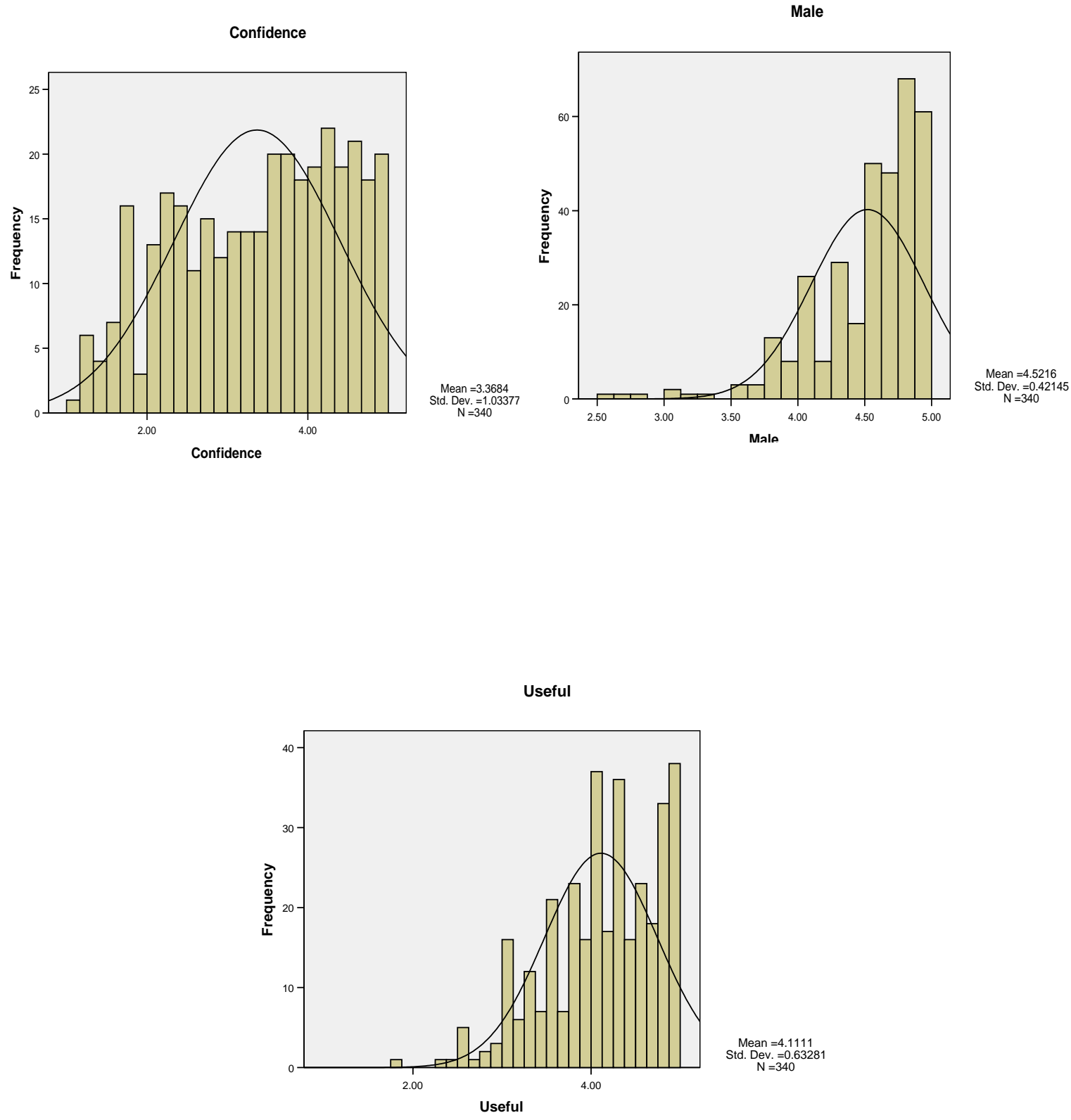


$\begin{array}{lcc}\begin{array}{l}\text { P. O Box 183 } \\ \text { 101 Old River Road } \\ \text { Salt Lick, KY 40371 }\end{array} & \text { Christine A. Perry } & \begin{array}{l}\text { Lappin Hall 201E } \\ \text { Morehead State University } \\ \text { Morehead, KY 40351 }\end{array} \\ \text { Home: (606) 683-4391 } & \text { Cell: (606) 336-2323 } & \text { Work: (606) 783-2935 }\end{array}$

\section{Educational Background}

University of Louisville/Appalachian Collaborative Center for Learning, Assessment, and Instruction in Mathematics (ACCLAIM)

Doctoral student (ABD), Department of Teaching and Learning, current Morehead State University, Morehead, KY

Rank I, Secondary Education, 1991

MA, Secondary Education, 1986

BS, Mathematics, 1975

\section{Certification}

Commonwealth of Kentucky

Mathematics Program Consultant, 2000 (Life)

Provisional High School Certificate, Grades 7-12, 1978 (Life)

\section{Professional Experience}

- Morehead State University, Morehead, KY

o Assistant Professor, Department of Mathematics and Computer Science, current

- Teacher-in-Residence, Department of Mathematics and Computer Science, Fall 2004 through Spring 2006

- Bath County School District, Owingsville, KY

o Mathematics Resource Teacher/ARSI Teacher Partner, 2000 - 2004

o Math Teacher, Bath County High School, 1997 to 2000

o Math Teacher, Bath County Middle School, 1989 to 1997

- Math Teacher, Salt Lick Elementary School, Grades 7 and 8, 1981 to 1989

o Migrant Math Teacher, Bath County High School, 1978 to 1981

\section{Professional Memberships}

- National Council of Teachers of Mathematics (NCTM)

- Kentucky Council of Teachers of Mathematics (KCTM)

- Association of Mathematics Teacher Educators (AMTE)

- Appalachian Association of Mathematics Teacher Educators (AAMTE), Charter Member

- Kentucky Education Association - Retired (KEA-R)

- National Education Association - Retired (NEA-R) 


\section{Professional Activities}

- Presenter - NCTM Annual Meeting, Atlanta, GA, March 21, 2007

- Presenter - NCTM Annual Meeting, St. Louis, MO, April 27, 2006

- AMSP PEP Grant - Partner with Bath County Schools; Grant awarded April 2006

- Teacher Partner - Appalachian Rural Systemic Initiative (ARSI), 2000 to 2004

- Authored HP Technology Grant for Bath County High School Science Department - awarded May 2004

- Authored AMSP PEP Grant for Bath and Clark County Schools partnering with Morehead State University - awarded Summer 2004.

- Publications - paper summarizing the five ideologies of mathematics education as discussed in the book, The Philosophy of Mathematics Education, by Paul Ernest, published as a Rural Mathematics Education Digest in the online ACCLAIM journal, Rural Mathematics Educator, Vol. 3, No. 1, March 2004

- Presenter - KCTM Conference, October 2003

- Resource Teacher - Kentucky Teacher Internship Program (KTIP), 2001

- Guest Instructor - Robinson Scholars Summer Program, University of Kentucky, 2001

- Scholastic Audit Team, Kentucky Department of Education, Office of Leadership and School Improvement, 2000

- Mathematics Department Head, Bath County High School, 1997 to 2000 